\title{
Amido-bridged $\mathrm{Cu}_{2} \mathrm{~N}_{2}$ diamond cores that minimize structural reorganization and facilitate reversible redox behavior between a $\mathrm{Cu}^{1} \mathrm{Cu}^{1}$ and a Class III delocalized $\mathrm{Cu}^{1.5} \mathrm{Cu}^{1.5}$ species.
}

\author{
Seth B. Harkins and Jonas C. Peters \\ Division of Chemistry and Chemical Engineering, Arnold and Mabel Beckman Laboratories of \\ Chemical Synthesis, California Institute of Technology, Pasadena, California 91125.
}

\section{Supporting Information}

S2

Graph 1: Plot of chemical shift (Hz) vs. $X_{2}$ from electron self-exchange experiments.

Graph 2: Plot of $\mathrm{W}_{2}, \mathrm{~W}_{3}$, and $\mathrm{W}_{23}$ vs. $X_{2}$ from electron self-exchange experiments.

Electronic structure calculation of $\mathbf{3}$

Figure 1: Cyclic voltammetry of $\mathbf{2}$ in THF and $\mathrm{CH}_{2} \mathrm{Cl}_{2}$.

Figure 2: X-band EPR spectrum $(9.38 \mathrm{GHz})$ of $\mathbf{3}$ at $5.2 \mathrm{~K}$ and simulation.

Figure 3: $\chi_{\mathrm{m}} \mathrm{T}$ versus $\mathrm{T}$ for $[(\mathrm{SNS}) \mathrm{Cu}]_{2}\left[\mathrm{~B}\left(\mathrm{C}_{6} \mathrm{H}_{3}\left(\mathrm{CF}_{3}\right)_{2}\right)_{4}\right], \mathbf{3}$

Figure 4. Fully labeled drawing of $[(\mathrm{SNS}) \mathrm{Cu}]_{2}, \mathbf{2}$

Figure 5. Drawing of $[(\mathrm{SNS}) \mathrm{Cu}]_{2}\left[\mathrm{~B}\left(\mathrm{C}_{8} \mathrm{H}_{3}\left(\mathrm{CF}_{3}\right)_{2}\right)_{4}\right], 3$

Figure 6. Fully labeled drawing of $[(\mathrm{SNS}) \mathrm{Cu}]_{2}\left[\mathrm{~B}\left(\mathrm{C}_{8} \mathrm{H}_{3}\left(\mathrm{CF}_{3}\right)_{2}\right)_{4}\right]$, anion omitted, 3

Figure 7. Fully labeled drawing of (SNS)CuCl, 4

Table 1. Crystal data and structure refinement for 2

Table 2. Atomic coordinates and equivalent isotropic displacement parameters for $\mathbf{2}$

Table 3. Bond lengths and angles for 2

Table 4. Anisotropic parameters for 2

Table 5. Hydrogen coordinates and isotropic displacement parameters for $\mathbf{2}$

Table 6. Crystal data and structure refinement for 3

Table 7. Atomic coordinates and equivalent isotropic displacement parameters for 3

Table 8 . Bond lengths and angles for $\mathbf{3}$

Table 9. Anisotropic parameters for $\mathbf{3}$

Table 10. Hydrogen coordinates and isotropic displacement parameters for $\mathbf{3}$

Table 11. Crystal data and structure refinement for $\mathbf{4}$

Table 12. Atomic coordinates and equivalent isotropic displacement parameters for 4

Table 13. Bond lengths and angles for 4 
S38 Table 15. Hydrogen coordinates and isotropic displacement parameters for 4 
Graph 1: Plot of chemical shift $(\mathrm{Hz})$ vs. $\chi_{2}$ from electron self-exchange experiments.

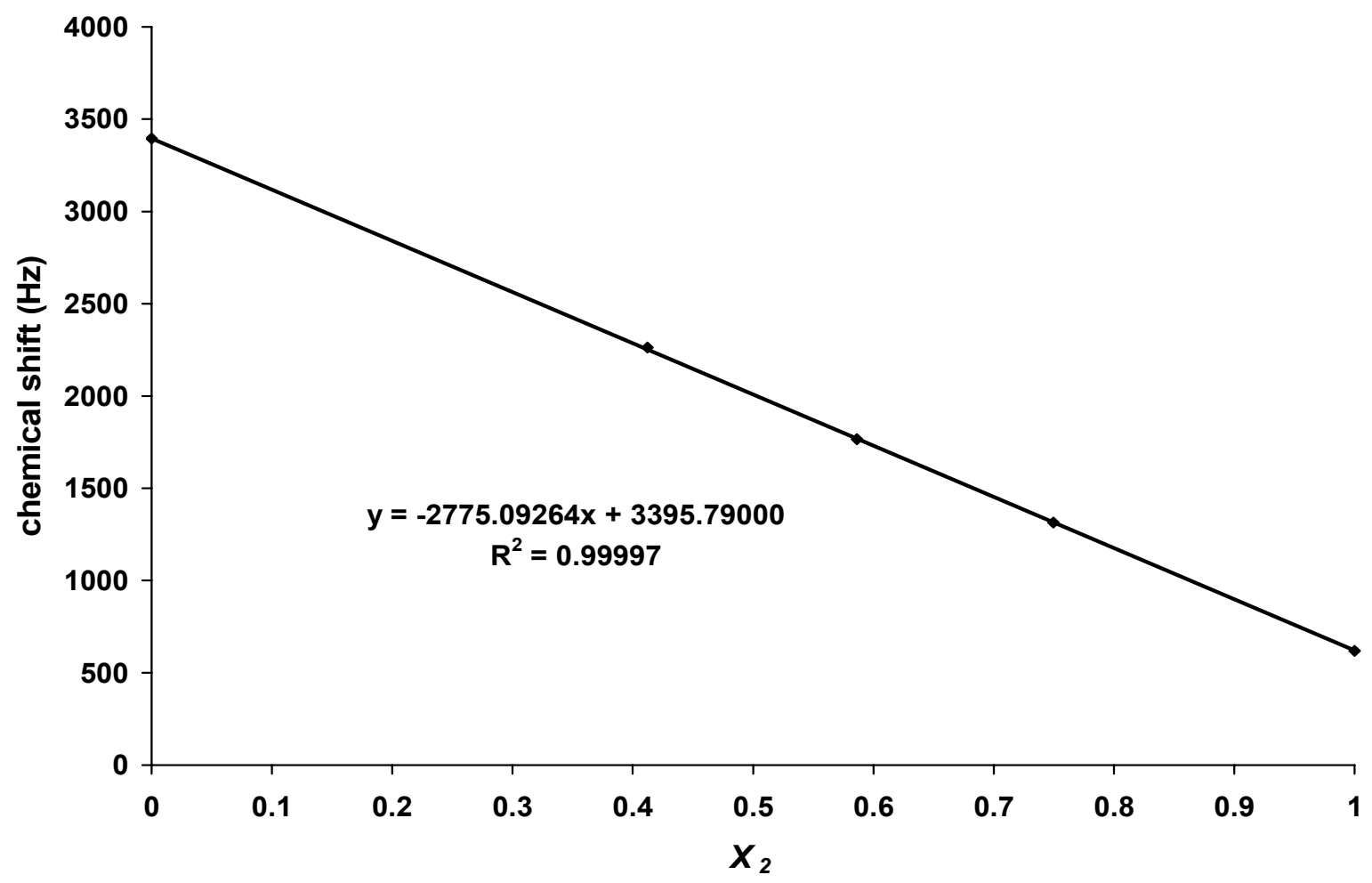

Graph 2: Plot of $W_{2}, W_{3}$, and $W_{23}$ vs. $\chi_{2}$ from electron self-exchange experiments.

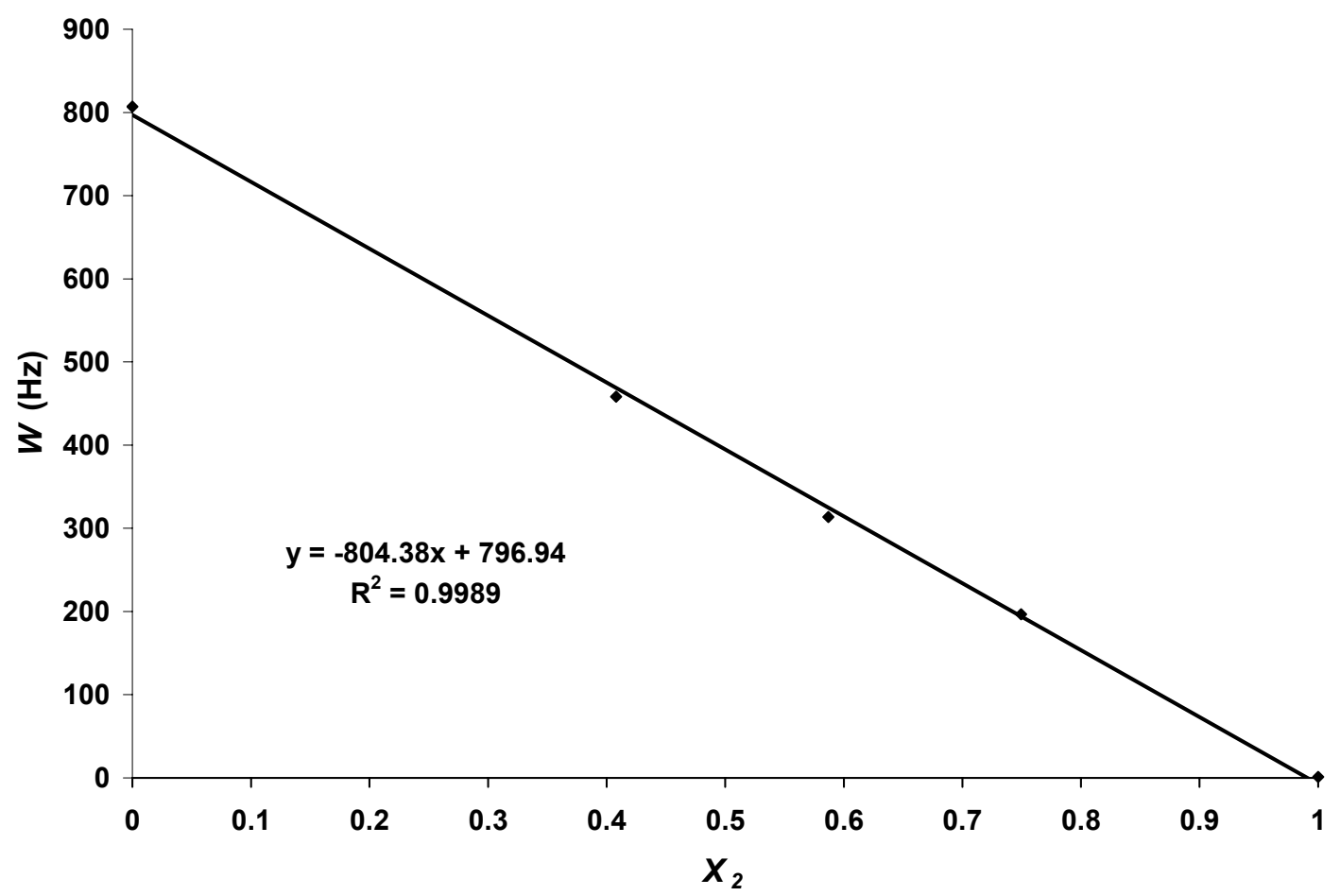


Electronic Structure Calculations. A hybrid density functional calculation was performed for complex 3 using Jaguar (version 4.0, release 20). The method used B3LYP with LACVP** as the basis set. A geometry optimization was carried out starting from coordinates based on the solid-state structure of cationic 3 that had been determined by an X-ray diffraction study. No symmetry constraints were imposed and the calculation was performed assuming a doublet electronic state. Optimization was considered converged when energy changes in successive iterations fell below $0.03 \mathrm{Kcal} / \mathrm{mol}$.

\begin{tabular}{|c|c|c|}
\hline Interatomic Distances $(\AA)$ & From X-ray & From DFT \\
\hline $\mathrm{Cu} 1-\mathrm{Cu} 2$ & $2.4724(4)$ & 2.502 \\
\hline $\mathrm{Cu} 1-\mathrm{N} 1$ & $2.0887(16)$ & 2.178 \\
\hline $\mathrm{Cu} 1-\mathrm{N} 2$ & $2.1011(17)$ & 2.171 \\
\hline $\mathrm{Cu} 1-\mathrm{S} 2$ & $2.2805(6)$ & 2.381 \\
\hline $\mathrm{Cu} 1-\mathrm{S} 3$ & $2.2805(6)$ & 2.382 \\
\hline Cu2-N1 & $2.0641(16)$ & 2.170 \\
\hline $\mathrm{Cu} 2-\mathrm{N} 2$ & $2.0568(16)$ & 2.164 \\
\hline $\mathrm{Cu} 2-\mathrm{S} 4$ & $2.2797(6)$ & 2.383 \\
\hline $\mathrm{Cu} 2-\mathrm{S} 1$ & $2.2834(6)$ & 2.387 \\
\hline \multicolumn{3}{|l|}{ Interatomic Angles (deg.) } \\
\hline Cu1-N1-Cu2 & $73.07(5)$ & 70.38 \\
\hline $\mathrm{Cu} 1-\mathrm{N} 2-\mathrm{Cu} 2$ & $72.96(5)$ & 70.36 \\
\hline N1-Cu1-N2 & $105.53(6)$ & 109.35 \\
\hline N1-Cu2-N2 & $108.09(6)$ & 109.91 \\
\hline S3-Cu1-S2 & $150.50(2)$ & 150.45 \\
\hline S1-Cu2-S4 & $150.71(2)$ & 150.16 \\
\hline
\end{tabular}
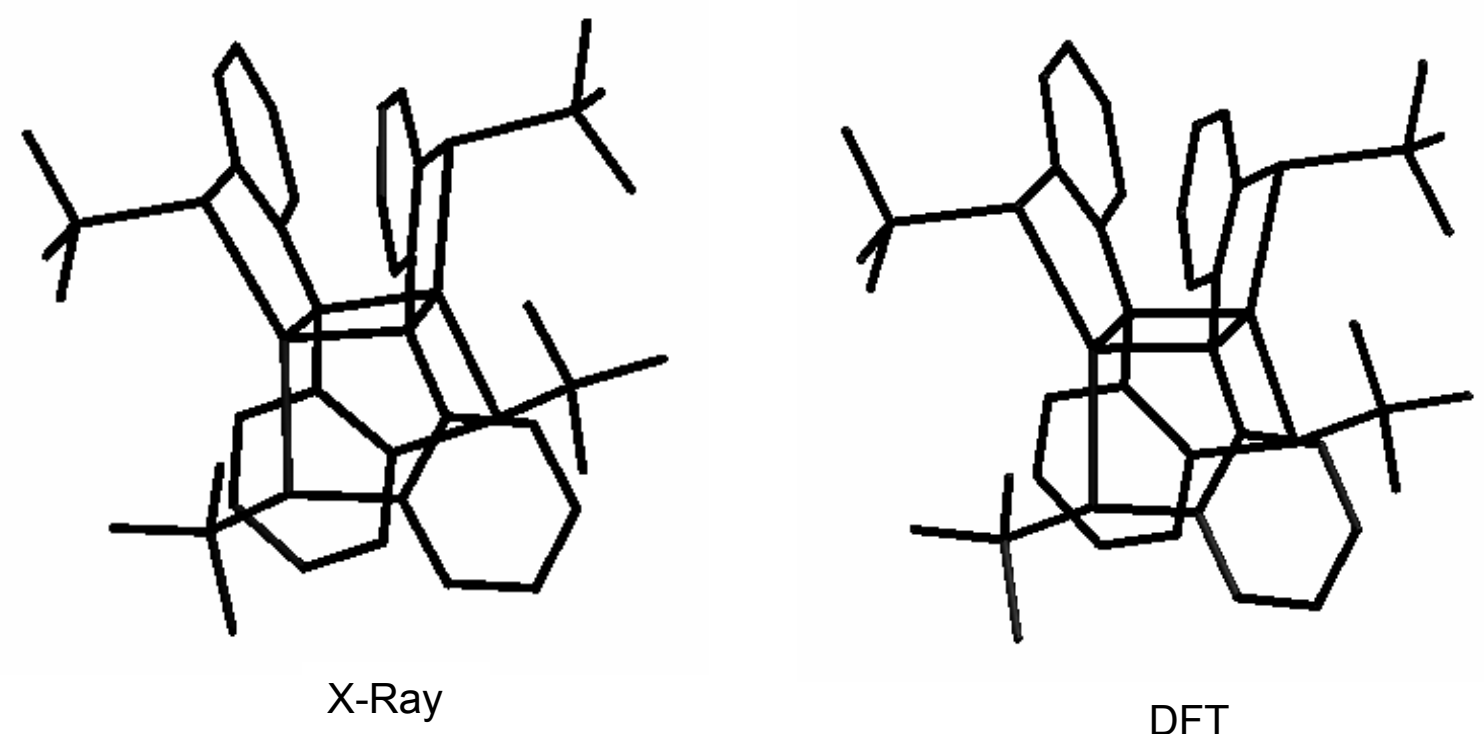

DFT 
Figure 1: Cyclic voltamogram of 2 in THF (top) (0.35 $\mathrm{M}\left[{ }^{\mathrm{n}} \mathrm{Bu}_{4} \mathrm{~N}\right]\left[\mathrm{PF}_{6}\right], 50 \mathrm{mV} / \mathrm{s}$ ) and $\mathrm{CH}_{2} \mathrm{Cl}_{2}$ $\left(0.30 \mathrm{M}\left[{ }^{\mathrm{n}} \mathrm{Bu}_{4} \mathrm{~N}\right]\left[\mathrm{PF}_{6}\right], 50 \mathrm{mV} / \mathrm{s}\right)$ (bottom), both referenced vs. $\mathrm{Fc}^{+} / \mathrm{Fc}$.
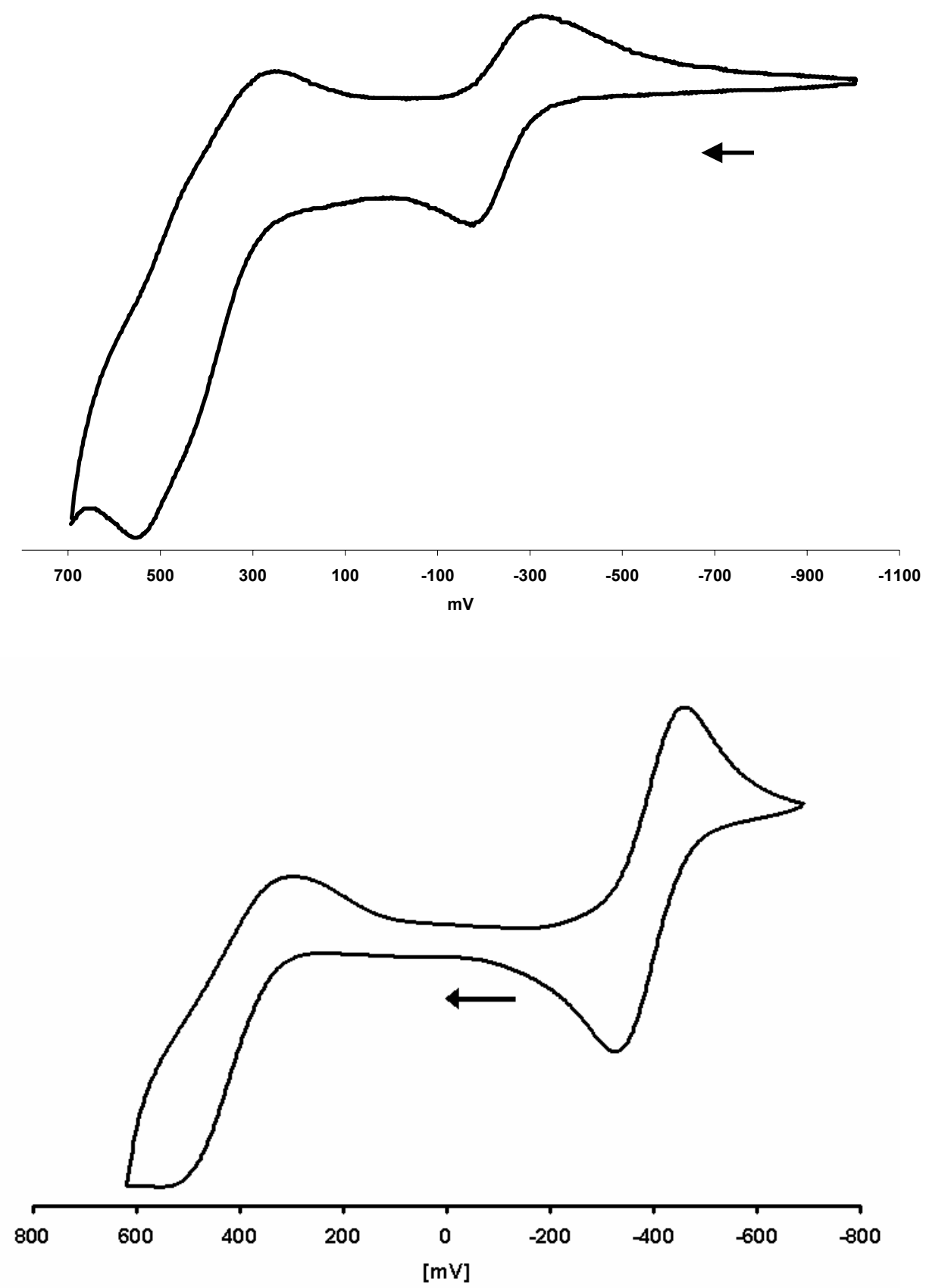
Figure 2: X-band EPR spectrum $(9.38 \mathrm{GHz})$ of 3 at $5.2 \mathrm{~K}$ in 2-methyltetrahydrofuran (upper trace). Parameters used to obtain the simulated spectrum (lower trace): $g_{1}=2.013, g_{2}=2.065, g_{3}$ $=2.069, A_{1}{ }^{C u}=10 \mathrm{G}, A_{2}{ }^{C u}=18.5 \mathrm{G}, A_{3}{ }^{C u}=44 \mathrm{G}$.

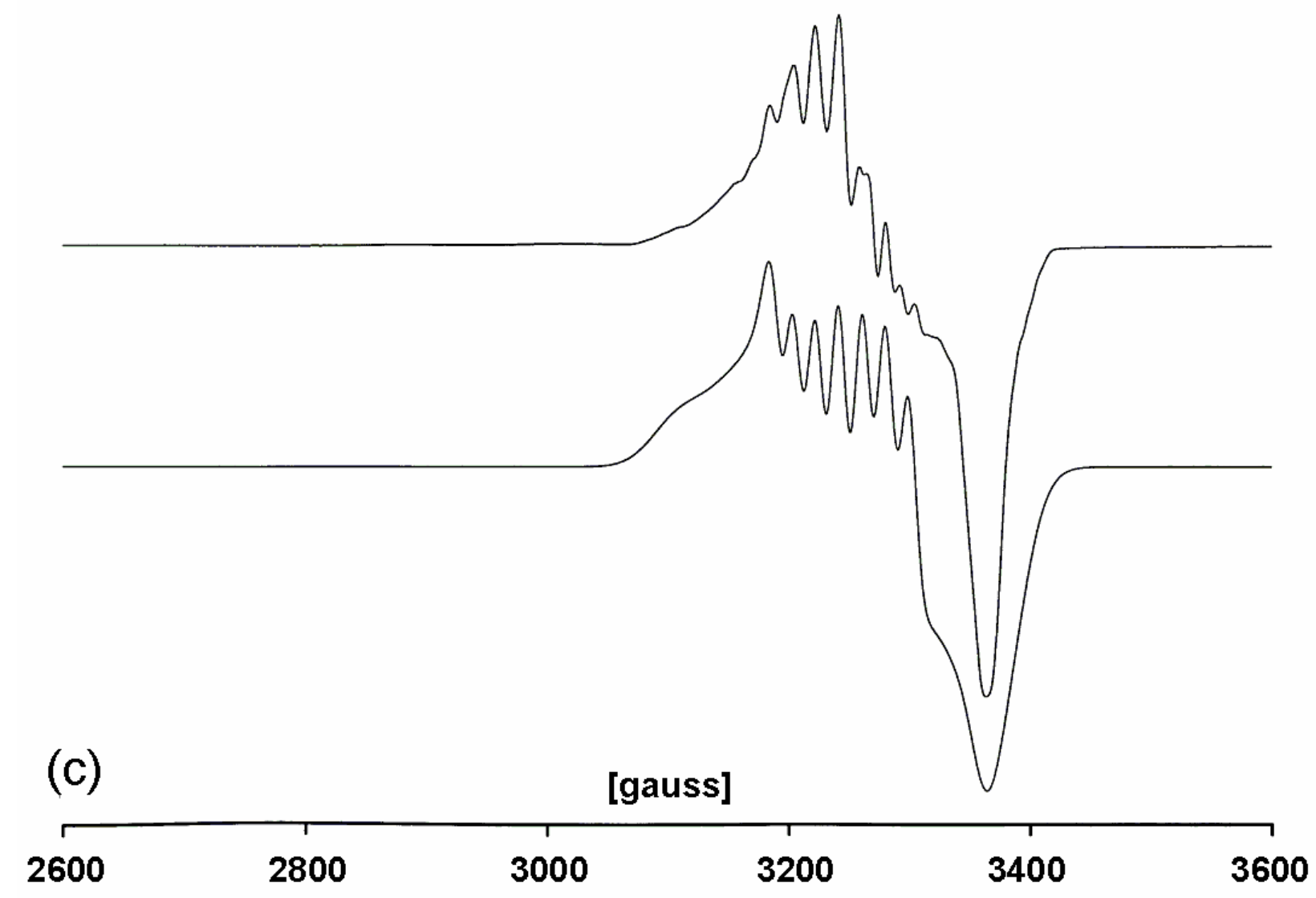


Figure 3: $\chi_{\mathrm{m}} \mathrm{T}$ versus $\mathrm{T}$ for $[(\mathrm{SNS}) \mathrm{Cu}]_{2}\left[\mathrm{~B}\left(\mathrm{C}_{6} \mathrm{H}_{3}\left(\mathrm{CF}_{3}\right)_{2}\right)_{4}\right], \mathbf{3}$.

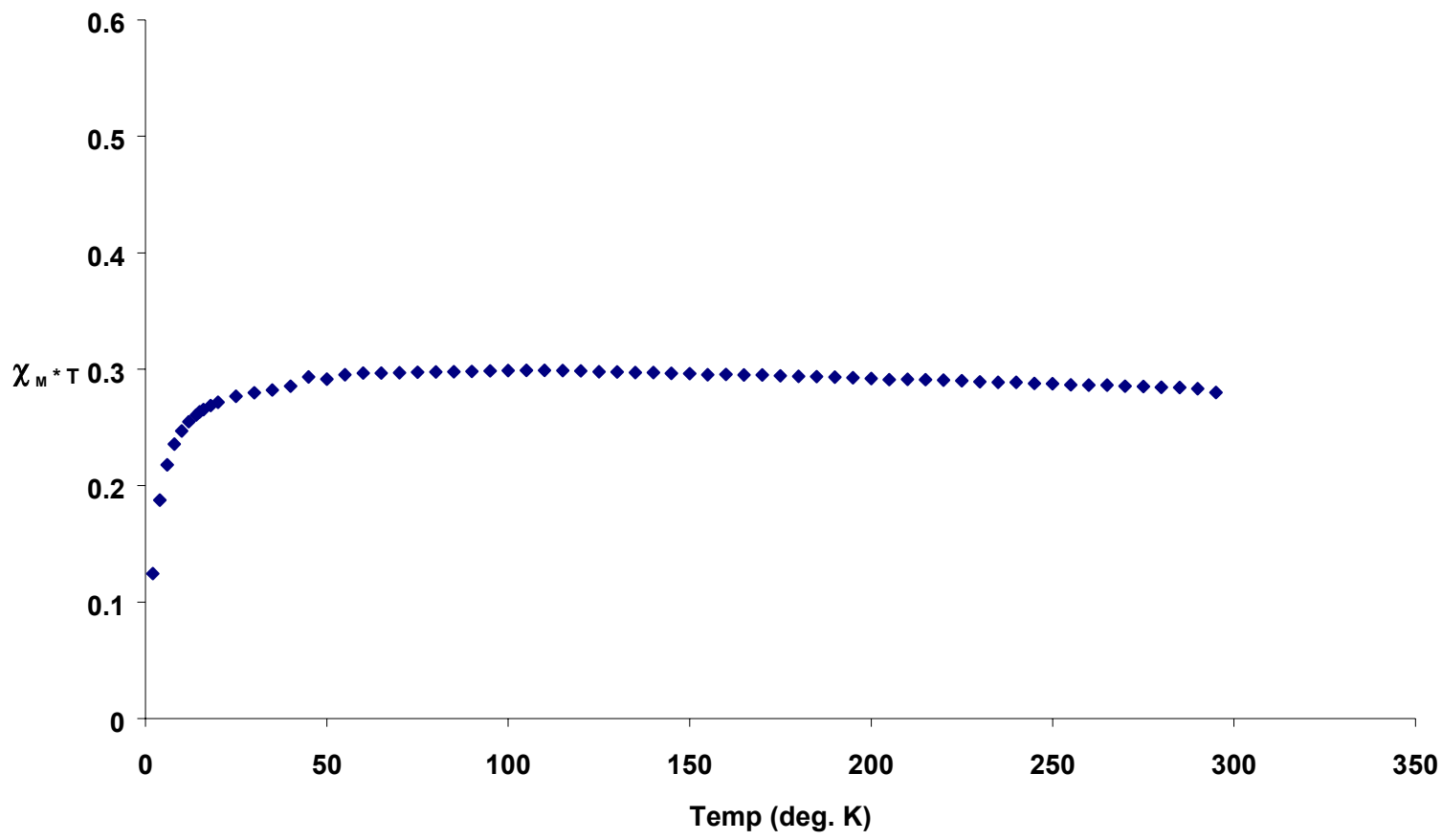


Figure 4. Fully labeled drawing of $[(\mathrm{SNS}) \mathrm{Cu}]_{2}, 2$. Hydrogens have been omitted for clarity.

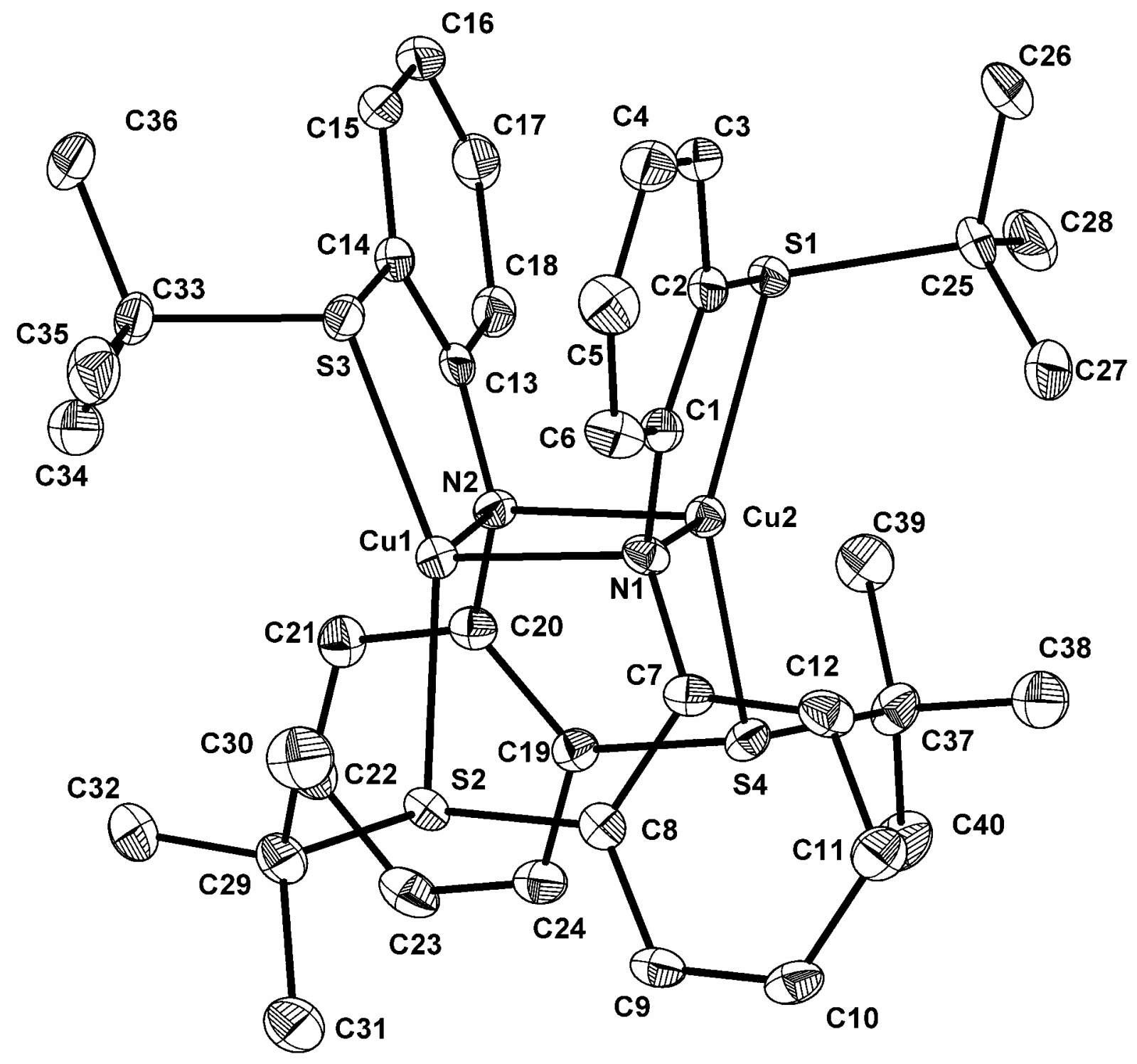


Figure 5. Drawing of $[(\mathrm{SNS}) \mathrm{Cu}]_{2}\left[\mathrm{~B}\left(\mathrm{C}_{6} \mathrm{H}_{3}\left(\mathrm{CF}_{3}\right)_{2}\right)_{4}\right]$, 3. Hydrogens and $1 / 2$ (ether) have been omitted for clarity.

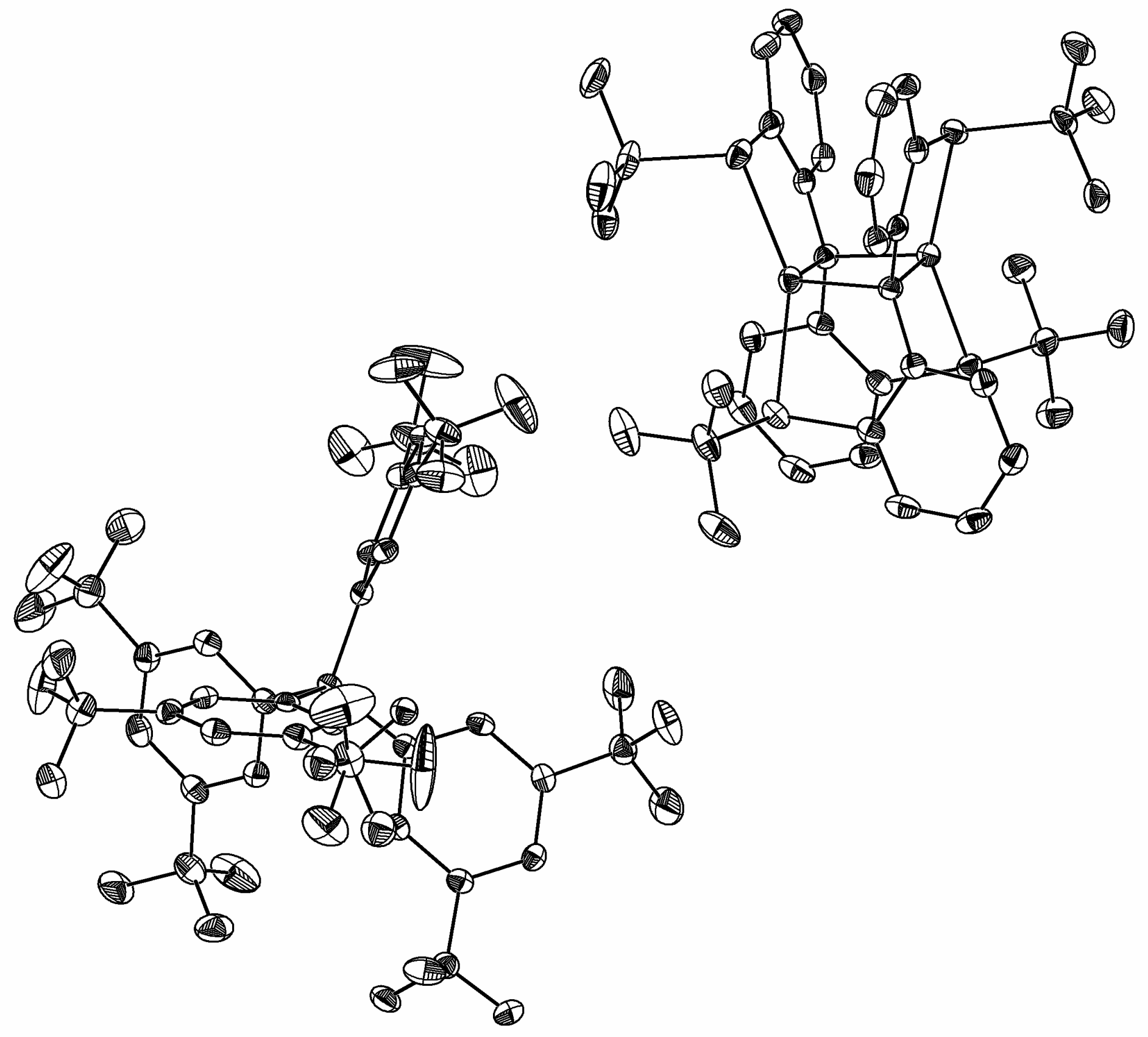


Figure 6. Fully labeled drawing of $[(\mathrm{SNS}) \mathrm{Cu}]_{2}\left[\mathrm{~B}\left(\mathrm{C}_{6} \mathrm{H}_{3}\left(\mathrm{CF}_{3}\right)_{2}\right)_{4}\right], 3$. Anion, hydrogens and $1 / 2$ (ether) have been omitted for clarity.

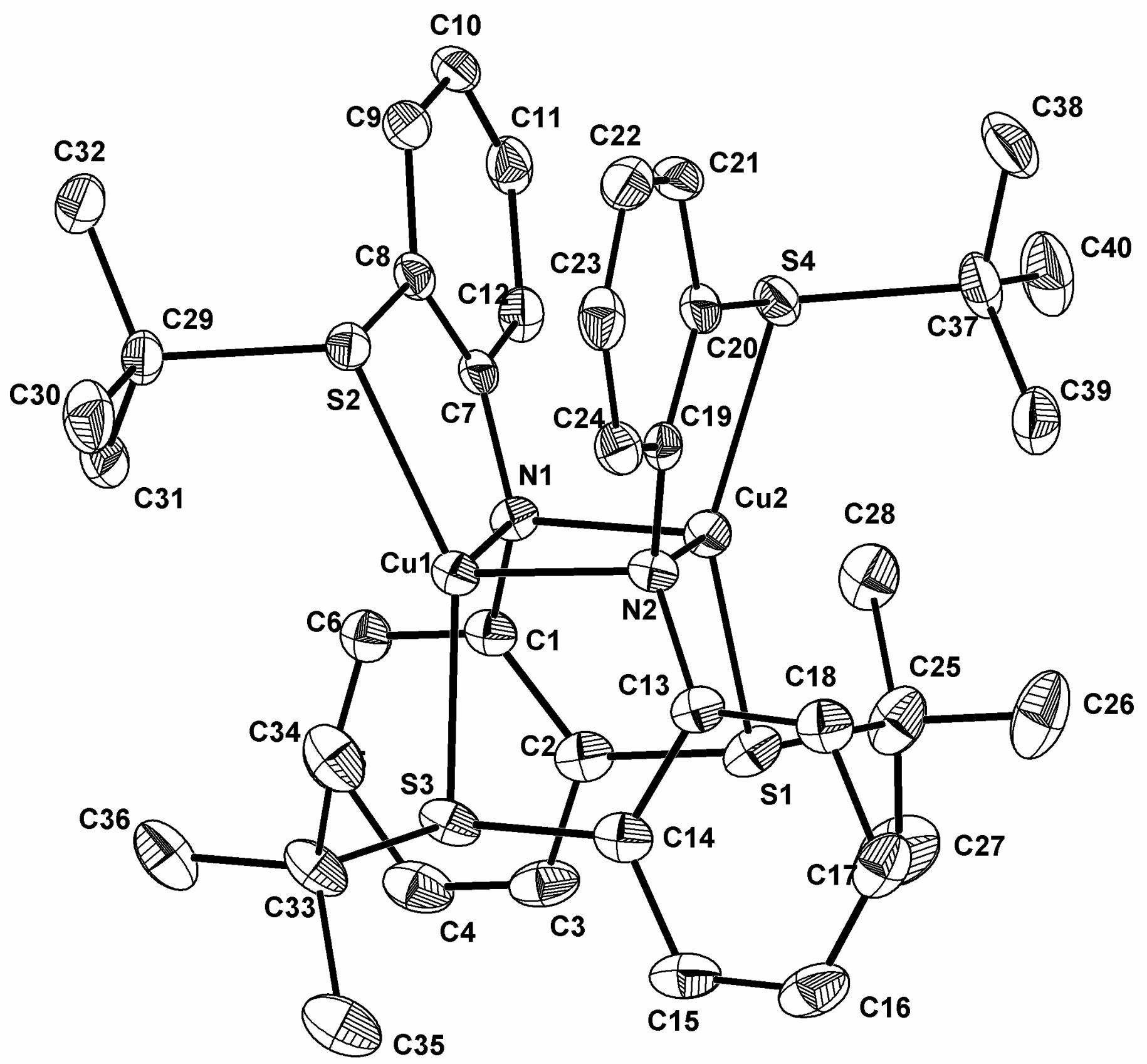


Figure 7. Drawing of (SNS)CuCl, 4. Hydrogens have been omitted for clarity.

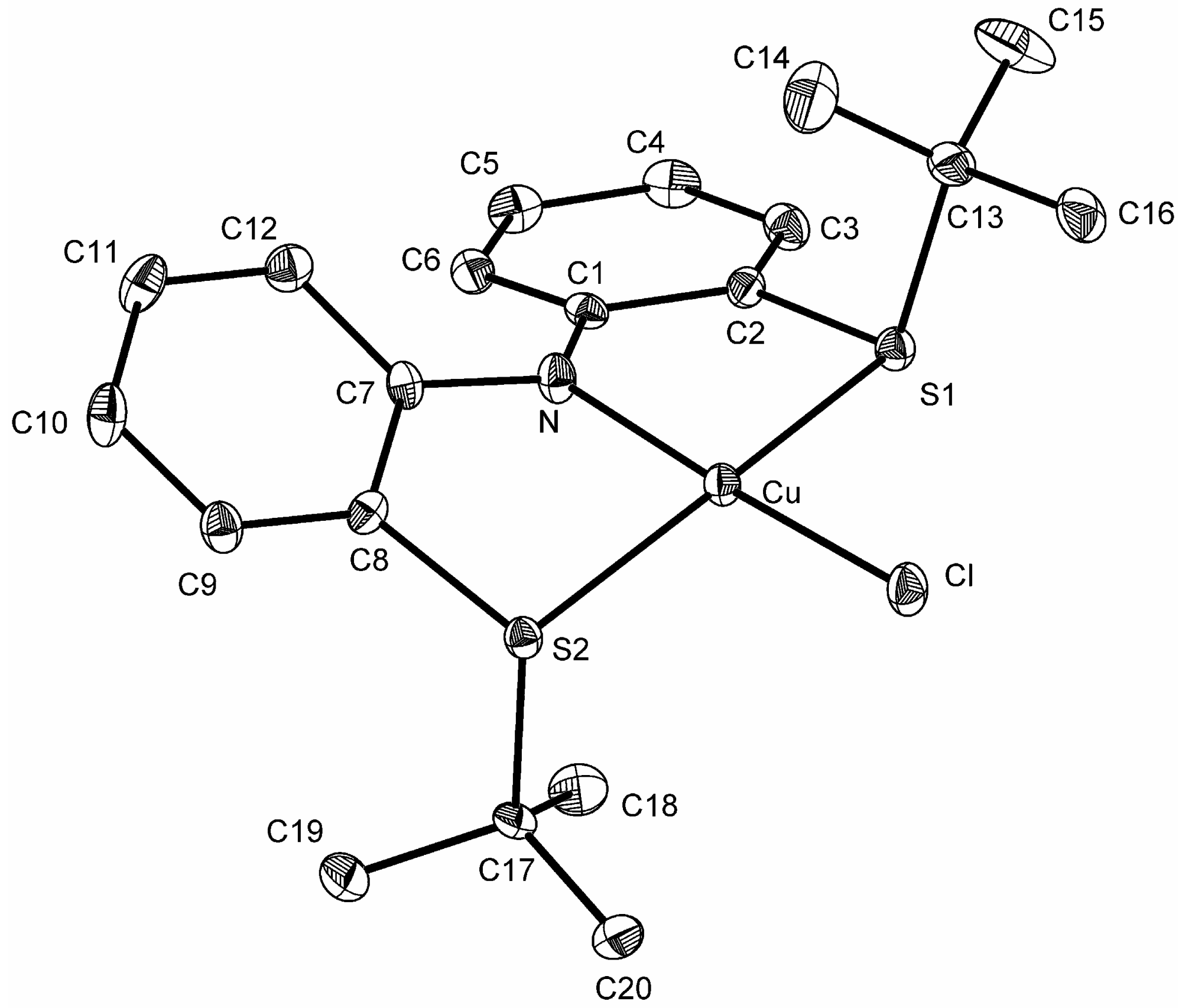


Table 1. Crystal data and structure refinement for $\mathbf{2}$.

Name of Complex

Empirical formula

Formula weight

Temperature

Wavelength

Crystal system

Crystal habit

Crystal color

Crystal size

Space group

Unit cell dimensions

Volume

Z

Density (calculated)

Absorption coefficient

$\mathrm{F}(000)$

Theta range for data collection

Index ranges

Reflections collected

Independent reflections

Completeness to theta $=28.40^{\circ}$

Absorption correction

Refinement method

Data / restraints / parameters

Goodness-of-fit on $\mathrm{F}^{2}$

Final $\mathrm{R}$ indices [I $>2 \operatorname{sigma}(\mathrm{I})]$

$\mathrm{R}$ indices (all data)

Largest diff. peak and hole

\author{
$\left[\left({ }^{\mathrm{t}} \mathrm{Bu}-\mathrm{SNS}\right) \mathrm{Cu}\right]_{2}$ \\ $\mathrm{C}_{40} \mathrm{H}_{52} \mathrm{Cu}_{2} \mathrm{~N}_{2} \mathrm{~S}_{4}$ \\ 816.206 \\ $98 \mathrm{~K}$ \\ $0.71073 \AA$ \\ Triclinic \\ rough block \\ yellow \\ $0.33 \times 0.28 \times 0.21 \mathrm{~mm}^{3}$ \\ P-1 (\#2) \\ $\mathrm{a}=10.2263(9) \AA$ \\ $\alpha=94.017(2)^{\circ}$. \\ $\mathrm{b}=10.6226(9) \AA$ \\ $\beta=90.634(2)^{\circ}$. \\ $\mathrm{c}=19.4884(17) \AA$ \\ $\gamma=107.305(1)^{\circ}$. \\ 2015.1(3) $\AA^{3}$ \\ 2 \\ $1.345 \mathrm{~g} / \mathrm{cm}^{3}$ \\ $1.292 \mathrm{~mm}^{-1}$ \\ 856 \\ 2.01 to $28.40^{\circ}$. \\ $-13<=\mathrm{h}<=13,-14<=\mathrm{k}<=13,-25<=\mathrm{l}<=25$ \\ 42010 \\ $9371[\mathrm{R}(\mathrm{int})=0.0507]$ \\ $92.4 \%$ \\ None \\ Full-matrix least-squares on $\mathrm{F}^{2}$ \\ 9371 / 0 / 445 \\ 1.464 \\ $\mathrm{R} 1=0.0303, \mathrm{wR} 2=0.0660$ \\ $\mathrm{R} 1=0.0416, \mathrm{wR} 2=0.0685$ \\ 0.679 and -0.300 e. $\AA^{-3}$
}

\section{Special Refinement Details}

Refinement of F2 against ALL reflections. The weighted R-factor $(w \mathrm{R})$ and goodness of fit (S) are based on F2, conventional R-factors (R) are based on F, with F set to zero for negative F2. The threshold expression of F2 $>2$ (F2) is used only for calculating R-factors(gt) etc. and is not relevant to the choice of reflections for refinement.

All esds (except the esd in the dihedral angle between two 1.s. planes) are estimated using the full covariance matrix. The cell esds are taken into account individually in the estimation of esds in distances, angles and torsion angles; correlations between esds in cell parameters are only used when they are defined by crystal symmetry. An approximate (isotropic) treatment of cell esds is used for estimating esds involving l.s. planes. 
Table 2. Atomic coordinates ( $\left.\mathrm{x} 10^{4}\right)$ and equivalent isotropic displacement parameters $\left(\AA^{2} \times 10^{3}\right)$ for 2. $U_{\text {eq }}$ is defined as one third of the trace of the orthogonalized $U^{i j}$ tensor.

\begin{tabular}{|c|c|c|c|c|}
\hline & $\mathrm{x}$ & $y$ & $\mathrm{z}$ & $\mathrm{U}_{\mathrm{eq}}$ \\
\hline$\overline{\mathrm{Cu}(1)}$ & $3175(1)$ & $6(1)$ & $2339(1)$ & $15(1)$ \\
\hline $\mathrm{Cu}(2)$ & $5267(1)$ & 2114(1) & 2544(1) & $14(1)$ \\
\hline $\mathrm{N}(1)$ & $5277(1)$ & $150(1)$ & 2439(1) & $15(1)$ \\
\hline $\mathrm{N}(2)$ & $3144(1)$ & 2003(1) & 2488(1) & $14(1)$ \\
\hline $\mathrm{S}(1)$ & $5912(1)$ & 2163(1) & $3675(1)$ & $16(1)$ \\
\hline$S(2)$ & $3277(1)$ & $-660(1)$ & $1212(1)$ & $16(1)$ \\
\hline $\mathrm{S}(3)$ & 2311(1) & $-270(1)$ & 3414(1) & $18(1)$ \\
\hline S(4) & $5433(1)$ & 2951(1) & $1496(1)$ & $16(1)$ \\
\hline $\mathrm{C}(1)$ & $5589(2)$ & $-333(2)$ & $3044(1)$ & $17(1)$ \\
\hline$C(2)$ & $5818(2)$ & $461(2)$ & $3674(1)$ & $17(1)$ \\
\hline$C(3)$ & $5904(2)$ & $-99(2)$ & 4294(1) & $22(1)$ \\
\hline $\mathrm{C}(4)$ & $5811(2)$ & $-1419(2)$ & $4307(1)$ & $26(1)$ \\
\hline$C(5)$ & $5643(2)$ & $-2201(2)$ & $3690(1)$ & $26(1)$ \\
\hline$C(6)$ & $5532(2)$ & $-1665(2)$ & 3073(1) & $22(1)$ \\
\hline$C(7)$ & $5830(2)$ & $-224(2)$ & $1833(1)$ & $16(1)$ \\
\hline $\mathrm{C}(8)$ & $5048(2)$ & $-570(2)$ & $1209(1)$ & $16(1)$ \\
\hline$C(9)$ & $5680(2)$ & $-767(2)$ & $595(1)$ & 19(1) \\
\hline$C(10)$ & $7047(2)$ & $-674(2)$ & $583(1)$ & $22(1)$ \\
\hline$C(11)$ & $7826(2)$ & $-374(2)$ & $1200(1)$ & $24(1)$ \\
\hline$C(12)$ & $7217(2)$ & $-148(2)$ & $1807(1)$ & $21(1)$ \\
\hline$C(13)$ & $2659(2)$ & $2322(2)$ & $3120(1)$ & $16(1)$ \\
\hline$C(14)$ & 2311(2) & 1395(2) & $3622(1)$ & $18(1)$ \\
\hline$C(15)$ & $2044(2)$ & 1782(2) & 4294(1) & $24(1)$ \\
\hline$C(16)$ & $2056(2)$ & $3063(2)$ & $4473(1)$ & $28(1)$ \\
\hline$C(17)$ & $2336(2)$ & 3977(2) & $3977(1)$ & $26(1)$ \\
\hline$C(18)$ & $2628(2)$ & $3620(2)$ & $3315(1)$ & $21(1)$ \\
\hline$C(19)$ & $3684(2)$ & 2895(2) & $1373(1)$ & $16(1)$ \\
\hline$C(20)$ & $2758(2)$ & $2507(2)$ & $1906(1)$ & $15(1)$ \\
\hline$C(21)$ & $1405(2)$ & 2534(2) & 1783(1) & $20(1)$ \\
\hline$C(22)$ & $982(2)$ & 2853(2) & 1161(1) & $23(1)$ \\
\hline$C(23)$ & $1893(2)$ & $3161(2)$ & $630(1)$ & $24(1)$ \\
\hline$C(24)$ & $3231(2)$ & $3174(2)$ & $737(1)$ & $20(1)$ \\
\hline$C(25)$ & $7756(2)$ & $3045(2)$ & $3880(1)$ & $24(1)$ \\
\hline$C(26)$ & $8155(2)$ & $2805(2)$ & $4604(1)$ & $32(1)$ \\
\hline$C(27)$ & $8642(2)$ & 2583(2) & $3352(1)$ & $35(1)$ \\
\hline$C(28)$ & $7857(2)$ & 4489(2) & $3844(1)$ & $34(1)$ \\
\hline$C(29)$ & $2352(2)$ & $-2455(2)$ & $1008(1)$ & $24(1)$ \\
\hline$C(30)$ & $2802(2)$ & $-3275(2)$ & $1519(1)$ & $32(1)$ \\
\hline$C(31)$ & $2615(2)$ & $-2896(2)$ & $273(1)$ & $34(1)$ \\
\hline$C(32)$ & $854(2)$ & $-2524(2)$ & $1065(1)$ & $33(1)$ \\
\hline$C(33)$ & $466(2)$ & $-1246(2)$ & $3363(1)$ & $22(1)$ \\
\hline$C(34)$ & $-285(2)$ & $-757(2)$ & 2811(1) & $26(1)$ \\
\hline$C(35)$ & $493(2)$ & $-2647(2)$ & $3174(1)$ & $32(1)$ \\
\hline$C(36)$ & $-202(2)$ & $-1162(2)$ & 4053(1) & $33(1)$ \\
\hline $\mathrm{C}(37)$ & 6381(2) & $4750(2)$ & 1614(1) & $20(1)$ \\
\hline C(38) & $7863(2)$ & $4767(2)$ & $1723(1)$ & $27(1)$ \\
\hline $\mathrm{C}(39)$ & $5876(2)$ & $5380(2)$ & $2239(1)$ & $26(1)$ \\
\hline $\mathrm{C}(40)$ & $6225(2)$ & 5453(2) & $975(1)$ & $28(1)$ \\
\hline
\end{tabular}


Table 3. Bond lengths $[\AA]$ and angles $\left[{ }^{\circ}\right]$ for 2.

\begin{tabular}{|c|c|c|c|}
\hline$\overline{\mathrm{Cu}(1)-\mathrm{Cu}(2)}$ & $2.5989(3)$ & $\mathrm{C}(22)-\mathrm{C}(23)$ & $1.392(3)$ \\
\hline $\mathrm{Cu}(1)-\mathrm{N}(1)$ & $2.1149(14)$ & $\mathrm{C}(22)-\mathrm{H}(22)$ & 0.9500 \\
\hline $\mathrm{Cu}(1)-\mathrm{N}(2)$ & $2.1303(14)$ & $\mathrm{C}(23)-\mathrm{C}(24)$ & $1.378(3)$ \\
\hline $\mathrm{Cu}(1)-\mathrm{S}(2)$ & $2.2730(5)$ & $\mathrm{C}(23)-\mathrm{H}(23)$ & 0.9500 \\
\hline $\mathrm{Cu}(1)-\mathrm{S}(3)$ & $2.2854(5)$ & $\mathrm{C}(24)-\mathrm{H}(24)$ & 0.9500 \\
\hline $\mathrm{Cu}(2)-\mathrm{N}(1)$ & $2.0850(14)$ & $C(25)-C(28)$ & $1.514(3)$ \\
\hline $\mathrm{Cu}(2)-\mathrm{N}(2)$ & $2.1395(14)$ & $C(25)-C(26)$ & $1.525(3)$ \\
\hline $\mathrm{Cu}(2)-\mathrm{S}(4)$ & $2.2735(5)$ & $C(25)-C(27)$ & $1.527(3)$ \\
\hline $\mathrm{Cu}(2)-\mathrm{S}(1)$ & $2.2853(5)$ & $\mathrm{C}(26)-\mathrm{H}(26 \mathrm{~A})$ & 0.9800 \\
\hline $\mathrm{N}(1)-\mathrm{C}(1)$ & $1.389(2)$ & $\mathrm{C}(26)-\mathrm{H}(26 \mathrm{~B})$ & 0.9800 \\
\hline $\mathrm{N}(1)-\mathrm{C}(7)$ & $1.398(2)$ & $\mathrm{C}(26)-\mathrm{H}(26 \mathrm{C})$ & 0.9800 \\
\hline $\mathrm{N}(2)-\mathrm{C}(13)$ & $1.390(2)$ & $\mathrm{C}(27)-\mathrm{H}(27 \mathrm{~A})$ & 0.9800 \\
\hline $\mathrm{N}(2)-\mathrm{C}(20)$ & $1.389(2)$ & $\mathrm{C}(27)-\mathrm{H}(27 \mathrm{~B})$ & 0.9800 \\
\hline $\mathrm{S}(1)-\mathrm{C}(2)$ & $1.7825(18)$ & $\mathrm{C}(27)-\mathrm{H}(27 \mathrm{C})$ & 0.9800 \\
\hline$S(1)-C(25)$ & $1.8637(18)$ & $\mathrm{C}(28)-\mathrm{H}(28 \mathrm{~A})$ & 0.9800 \\
\hline $\mathrm{S}(2)-\mathrm{C}(8)$ & $1.7856(18)$ & $\mathrm{C}(28)-\mathrm{H}(28 \mathrm{~B})$ & 0.9800 \\
\hline$S(2)-C(29)$ & $1.8707(18)$ & $\mathrm{C}(28)-\mathrm{H}(28 \mathrm{C})$ & 0.9800 \\
\hline $\mathrm{S}(3)-\mathrm{C}(14)$ & $1.7870(19)$ & $\mathrm{C}(29)-\mathrm{C}(32)$ & $1.517(3)$ \\
\hline $\mathrm{S}(3)-\mathrm{C}(33)$ & $1.8610(18)$ & $C(29)-C(30)$ & $1.521(3)$ \\
\hline$S(4)-C(19)$ & $1.7848(18)$ & $C(29)-C(31)$ & $1.528(3)$ \\
\hline$S(4)-C(37)$ & $1.8641(18)$ & $\mathrm{C}(30)-\mathrm{H}(30 \mathrm{~A})$ & 0.9800 \\
\hline$C(1)-C(6)$ & $1.403(3)$ & $\mathrm{C}(30)-\mathrm{H}(30 \mathrm{~B})$ & 0.9800 \\
\hline $\mathrm{C}(1)-\mathrm{C}(2)$ & $1.416(2)$ & $\mathrm{C}(30)-\mathrm{H}(30 \mathrm{C})$ & 0.9800 \\
\hline $\mathrm{C}(2)-\mathrm{C}(3)$ & $1.396(2)$ & $\mathrm{C}(31)-\mathrm{H}(31 \mathrm{~A})$ & 0.9800 \\
\hline$C(3)-C(4)$ & $1.379(3)$ & $\mathrm{C}(31)-\mathrm{H}(31 \mathrm{~B})$ & 0.9800 \\
\hline $\mathrm{C}(3)-\mathrm{H}(3)$ & 0.9500 & $\mathrm{C}(31)-\mathrm{H}(31 \mathrm{C})$ & 0.9800 \\
\hline$C(4)-C(5)$ & 1.391(3) & $\mathrm{C}(32)-\mathrm{H}(32 \mathrm{~A})$ & 0.9800 \\
\hline $\mathrm{C}(4)-\mathrm{H}(4)$ & 0.9500 & $\mathrm{C}(32)-\mathrm{H}(32 \mathrm{~B})$ & 0.9800 \\
\hline$C(5)-C(6)$ & $1.385(3)$ & $\mathrm{C}(32)-\mathrm{H}(32 \mathrm{C})$ & 0.9800 \\
\hline $\mathrm{C}(5)-\mathrm{H}(5)$ & 0.9500 & $C(33)-C(35)$ & $1.516(3)$ \\
\hline $\mathrm{C}(6)-\mathrm{H}(6)$ & 0.9500 & $C(33)-C(36)$ & $1.524(3)$ \\
\hline$C(7)-C(12)$ & $1.398(2)$ & $C(33)-C(34)$ & $1.521(2)$ \\
\hline$C(7)-C(8)$ & $1.413(2)$ & $\mathrm{C}(34)-\mathrm{H}(34 \mathrm{~A})$ & 0.9800 \\
\hline $\mathrm{C}(8)-\mathrm{C}(9)$ & $1.398(2)$ & $\mathrm{C}(34)-\mathrm{H}(34 \mathrm{~B})$ & 0.9800 \\
\hline$C(9)-C(10)$ & $1.372(3)$ & $\mathrm{C}(34)-\mathrm{H}(34 \mathrm{C})$ & 0.9800 \\
\hline $\mathrm{C}(9)-\mathrm{H}(9)$ & 0.9500 & $\mathrm{C}(35)-\mathrm{H}(35 \mathrm{~A})$ & 0.9800 \\
\hline$C(10)-C(11)$ & $1.397(3)$ & $\mathrm{C}(35)-\mathrm{H}(35 \mathrm{~B})$ & 0.9800 \\
\hline $\mathrm{C}(10)-\mathrm{H}(10)$ & 0.9500 & $\mathrm{C}(35)-\mathrm{H}(35 \mathrm{C})$ & 0.9800 \\
\hline C(11)-C(12) & $1.382(3)$ & $\mathrm{C}(36)-\mathrm{H}(36 \mathrm{~A})$ & 0.9800 \\
\hline $\mathrm{C}(11)-\mathrm{H}(11)$ & 0.9500 & $\mathrm{C}(36)-\mathrm{H}(36 \mathrm{~B})$ & 0.9800 \\
\hline $\mathrm{C}(12)-\mathrm{H}(12)$ & 0.9500 & $\mathrm{C}(36)-\mathrm{H}(36 \mathrm{C})$ & 0.9800 \\
\hline $\mathrm{C}(13)-\mathrm{C}(14)$ & $1.410(3)$ & $\mathrm{C}(37)-\mathrm{C}(39)$ & $1.518(3)$ \\
\hline $\mathrm{C}(13)-\mathrm{C}(18)$ & $1.414(3)$ & $\mathrm{C}(37)-\mathrm{C}(38)$ & $1.522(3)$ \\
\hline C(14)-C(15) & $1.399(2)$ & $\mathrm{C}(37)-\mathrm{C}(40)$ & $1.527(3)$ \\
\hline C(15)-C(16) & $1.378(3)$ & $\mathrm{C}(38)-\mathrm{H}(38 \mathrm{~A})$ & 0.9800 \\
\hline $\mathrm{C}(15)-\mathrm{H}(15)$ & 0.9500 & $\mathrm{C}(38)-\mathrm{H}(38 \mathrm{~B})$ & 0.9800 \\
\hline$C(16)-C(17)$ & $1.389(3)$ & $\mathrm{C}(38)-\mathrm{H}(38 \mathrm{C})$ & 0.9800 \\
\hline $\mathrm{C}(16)-\mathrm{H}(16)$ & 0.9500 & $\mathrm{C}(39)-\mathrm{H}(39 \mathrm{~A})$ & 0.9800 \\
\hline $\mathrm{C}(17)-\mathrm{C}(18)$ & $1.380(3)$ & $\mathrm{C}(39)-\mathrm{H}(39 \mathrm{~B})$ & 0.9800 \\
\hline $\mathrm{C}(17)-\mathrm{H}(17)$ & 0.9500 & $\mathrm{C}(39)-\mathrm{H}(39 \mathrm{C})$ & 0.9800 \\
\hline $\mathrm{C}(18)-\mathrm{H}(18)$ & 0.9500 & $\mathrm{C}(40)-\mathrm{H}(40 \mathrm{~A})$ & 0.9800 \\
\hline C(19)-C(24) & $1.400(2)$ & $\mathrm{C}(40)-\mathrm{H}(40 \mathrm{~B})$ & 0.9800 \\
\hline C(19)-C(20) & $1.413(2)$ & $\mathrm{C}(40)-\mathrm{H}(40 \mathrm{C})$ & 0.9800 \\
\hline C(20)-C(21) & $1.410(2)$ & $\mathrm{N}(1)-\mathrm{Cu}(1)-\mathrm{N}(2)$ & $103.88(5)$ \\
\hline$C(21)-C(22)$ & $1.380(3)$ & $\mathrm{N}(1)-\mathrm{Cu}(1)-\mathrm{S}(2)$ & $87.36(4)$ \\
\hline $\mathrm{C}(21)-\mathrm{H}(21)$ & 0.9500 & $\mathrm{~N}(2)-\mathrm{Cu}(1)-\mathrm{S}(2)$ & $112.76(4)$ \\
\hline
\end{tabular}




\begin{tabular}{|c|c|c|c|}
\hline $\mathrm{N}(1)-\mathrm{Cu}(1)-\mathrm{S}(3)$ & $106.53(4)$ & $\mathrm{C}(10)-\mathrm{C}(9)-\mathrm{H}(9)$ & 119.2 \\
\hline $\mathrm{N}(2)-\mathrm{Cu}(1)-\mathrm{S}(3)$ & $86.87(4)$ & $\mathrm{C}(8)-\mathrm{C}(9)-\mathrm{H}(9)$ & 119.2 \\
\hline $\mathrm{S}(2)-\mathrm{Cu}(1)-\mathrm{S}(3)$ & 152.921(19) & $\mathrm{C}(9)-\mathrm{C}(10)-\mathrm{C}(11)$ & $119.18(17)$ \\
\hline $\mathrm{N}(1)-\mathrm{Cu}(2)-\mathrm{N}(2)$ & $104.59(5)$ & $\mathrm{C}(9)-\mathrm{C}(10)-\mathrm{H}(10)$ & 120.4 \\
\hline $\mathrm{N}(1)-\mathrm{Cu}(2)-\mathrm{S}(4)$ & 109.94(4) & $\mathrm{C}(11)-\mathrm{C}(10)-\mathrm{H}(10)$ & 120.4 \\
\hline $\mathrm{N}(2)-\mathrm{Cu}(2)-\mathrm{S}(4)$ & $86.80(4)$ & $\mathrm{C}(12)-\mathrm{C}(11)-\mathrm{C}(10)$ & $119.65(17)$ \\
\hline $\mathrm{N}(1)-\mathrm{Cu}(2)-\mathrm{S}(1)$ & $87.87(4)$ & $\mathrm{C}(12)-\mathrm{C}(11)-\mathrm{H}(11)$ & 120.2 \\
\hline $\mathrm{N}(2)-\mathrm{Cu}(2)-\mathrm{S}(1)$ & $108.49(4)$ & $\mathrm{C}(10)-\mathrm{C}(11)-\mathrm{H}(11)$ & 120.2 \\
\hline $\mathrm{S}(4)-\mathrm{Cu}(2)-\mathrm{S}(1)$ & 153.233(19) & $C(11)-C(12)-C(7)$ & $122.42(17)$ \\
\hline $\mathrm{C}(1)-\mathrm{N}(1)-\mathrm{C}(7)$ & $117.51(14)$ & $\mathrm{C}(11)-\mathrm{C}(12)-\mathrm{H}(12)$ & 118.8 \\
\hline $\mathrm{C}(1)-\mathrm{N}(1)-\mathrm{Cu}(2)$ & $114.38(11)$ & $\mathrm{C}(7)-\mathrm{C}(12)-\mathrm{H}(12)$ & 118.8 \\
\hline $\mathrm{C}(7)-\mathrm{N}(1)-\mathrm{Cu}(2)$ & $115.46(11)$ & $\mathrm{N}(2)-\mathrm{C}(13)-\mathrm{C}(14)$ & $121.02(16)$ \\
\hline $\mathrm{C}(1)-\mathrm{N}(1)-\mathrm{Cu}(1)$ & $112.31(11)$ & $\mathrm{N}(2)-\mathrm{C}(13)-\mathrm{C}(18)$ & $121.74(16)$ \\
\hline $\mathrm{C}(7)-\mathrm{N}(1)-\mathrm{Cu}(1)$ & $113.93(11)$ & $\mathrm{C}(14)-\mathrm{C}(13)-\mathrm{C}(18)$ & $116.92(16)$ \\
\hline $\mathrm{Cu}(2)-\mathrm{N}(1)-\mathrm{Cu}(1)$ & $76.45(5)$ & $C(15)-C(14)-C(13)$ & $120.74(17)$ \\
\hline $\mathrm{C}(13)-\mathrm{N}(2)-\mathrm{C}(20)$ & $118.43(14)$ & $C(15)-C(14)-S(3)$ & $119.44(15)$ \\
\hline $\mathrm{C}(13)-\mathrm{N}(2)-\mathrm{Cu}(1)$ & 114.34(11) & $\mathrm{C}(13)-\mathrm{C}(14)-\mathrm{S}(3)$ & $119.71(13)$ \\
\hline $\mathrm{C}(20)-\mathrm{N}(2)-\mathrm{Cu}(1)$ & $114.60(11)$ & $\mathrm{C}(16)-\mathrm{C}(15)-\mathrm{C}(14)$ & $120.83(19)$ \\
\hline $\mathrm{C}(13)-\mathrm{N}(2)-\mathrm{Cu}(2)$ & $112.65(10)$ & $\mathrm{C}(16)-\mathrm{C}(15)-\mathrm{H}(15)$ & 119.6 \\
\hline $\mathrm{C}(20)-\mathrm{N}(2)-\mathrm{Cu}(2)$ & $114.21(11)$ & $\mathrm{C}(14)-\mathrm{C}(15)-\mathrm{H}(15)$ & 119.6 \\
\hline $\mathrm{Cu}(1)-\mathrm{N}(2)-\mathrm{Cu}(2)$ & $74.99(5)$ & $C(15)-C(16)-C(17)$ & $119.21(18)$ \\
\hline$C(2)-S(1)-C(25)$ & 103.62(9) & $\mathrm{C}(15)-\mathrm{C}(16)-\mathrm{H}(16)$ & 120.4 \\
\hline $\mathrm{C}(2)-\mathrm{S}(1)-\mathrm{Cu}(2)$ & $96.72(6)$ & $\mathrm{C}(17)-\mathrm{C}(16)-\mathrm{H}(16)$ & 120.4 \\
\hline $\mathrm{C}(25)-\mathrm{S}(1)-\mathrm{Cu}(2)$ & $114.49(6)$ & $\mathrm{C}(18)-\mathrm{C}(17)-\mathrm{C}(16)$ & $120.76(19)$ \\
\hline $\mathrm{C}(8)-\mathrm{S}(2)-\mathrm{C}(29)$ & $104.35(8)$ & $\mathrm{C}(18)-\mathrm{C}(17)-\mathrm{H}(17)$ & 119.6 \\
\hline $\mathrm{C}(8)-\mathrm{S}(2)-\mathrm{Cu}(1)$ & $98.12(6)$ & $\mathrm{C}(16)-\mathrm{C}(17)-\mathrm{H}(17)$ & 119.6 \\
\hline $\mathrm{C}(29)-\mathrm{S}(2)-\mathrm{Cu}(1)$ & $112.80(6)$ & $\mathrm{C}(17)-\mathrm{C}(18)-\mathrm{C}(13)$ & $121.40(18)$ \\
\hline $\mathrm{C}(14)-\mathrm{S}(3)-\mathrm{C}(33)$ & 104.44(8) & $\mathrm{C}(17)-\mathrm{C}(18)-\mathrm{H}(18)$ & 119.3 \\
\hline $\mathrm{C}(14)-\mathrm{S}(3)-\mathrm{Cu}(1)$ & $97.72(6)$ & $\mathrm{C}(13)-\mathrm{C}(18)-\mathrm{H}(18)$ & 119.3 \\
\hline $\mathrm{C}(33)-\mathrm{S}(3)-\mathrm{Cu}(1)$ & $110.95(6)$ & $\mathrm{C}(24)-\mathrm{C}(19)-\mathrm{C}(20)$ & $120.70(16)$ \\
\hline C(19)-S(4)-C(37) & $104.45(8)$ & $C(24)-C(19)-S(4)$ & $119.53(14)$ \\
\hline $\mathrm{C}(19)-\mathrm{S}(4)-\mathrm{Cu}(2)$ & $98.14(6)$ & $\mathrm{C}(20)-\mathrm{C}(19)-\mathrm{S}(4)$ & $119.71(13)$ \\
\hline $\mathrm{C}(37)-\mathrm{S}(4)-\mathrm{Cu}(2)$ & $108.17(6)$ & $\mathrm{N}(2)-\mathrm{C}(20)-\mathrm{C}(21)$ & $122.44(16)$ \\
\hline $\mathrm{N}(1)-\mathrm{C}(1)-\mathrm{C}(6)$ & $121.45(16)$ & $\mathrm{N}(2)-\mathrm{C}(20)-\mathrm{C}(19)$ & $120.65(15)$ \\
\hline $\mathrm{N}(1)-\mathrm{C}(1)-\mathrm{C}(2)$ & $121.03(16)$ & $\mathrm{C}(21)-\mathrm{C}(20)-\mathrm{C}(19)$ & $116.61(16)$ \\
\hline$C(6)-C(1)-C(2)$ & $117.12(16)$ & $\mathrm{C}(22)-\mathrm{C}(21)-\mathrm{C}(20)$ & $121.99(17)$ \\
\hline $\mathrm{C}(3)-\mathrm{C}(2)-\mathrm{C}(1)$ & $120.31(17)$ & $\mathrm{C}(22)-\mathrm{C}(21)-\mathrm{H}(21)$ & 119.0 \\
\hline $\mathrm{C}(3)-\mathrm{C}(2)-\mathrm{S}(1)$ & $120.15(14)$ & $\mathrm{C}(20)-\mathrm{C}(21)-\mathrm{H}(21)$ & 119.0 \\
\hline$C(1)-C(2)-S(1)$ & $119.47(13)$ & $\mathrm{C}(21)-\mathrm{C}(22)-\mathrm{C}(23)$ & $120.39(18)$ \\
\hline$C(4)-C(3)-C(2)$ & $121.18(18)$ & $\mathrm{C}(21)-\mathrm{C}(22)-\mathrm{H}(22)$ & 119.8 \\
\hline $\mathrm{C}(4)-\mathrm{C}(3)-\mathrm{H}(3)$ & 119.4 & $\mathrm{C}(23)-\mathrm{C}(22)-\mathrm{H}(22)$ & 119.8 \\
\hline $\mathrm{C}(2)-\mathrm{C}(3)-\mathrm{H}(3)$ & 119.4 & $\mathrm{C}(24)-\mathrm{C}(23)-\mathrm{C}(22)$ & $119.20(17)$ \\
\hline$C(3)-C(4)-C(5)$ & 119.24(18) & $\mathrm{C}(24)-\mathrm{C}(23)-\mathrm{H}(23)$ & 120.4 \\
\hline $\mathrm{C}(3)-\mathrm{C}(4)-\mathrm{H}(4)$ & 120.4 & $\mathrm{C}(22)-\mathrm{C}(23)-\mathrm{H}(23)$ & 120.4 \\
\hline $\mathrm{C}(5)-\mathrm{C}(4)-\mathrm{H}(4)$ & 120.4 & $\mathrm{C}(23)-\mathrm{C}(24)-\mathrm{C}(19)$ & $120.90(18)$ \\
\hline$C(6)-C(5)-C(4)$ & $120.18(18)$ & $\mathrm{C}(23)-\mathrm{C}(24)-\mathrm{H}(24)$ & 119.5 \\
\hline $\mathrm{C}(6)-\mathrm{C}(5)-\mathrm{H}(5)$ & 119.9 & $\mathrm{C}(19)-\mathrm{C}(24)-\mathrm{H}(24)$ & 119.5 \\
\hline $\mathrm{C}(4)-\mathrm{C}(5)-\mathrm{H}(5)$ & 119.9 & $\mathrm{C}(28)-\mathrm{C}(25)-\mathrm{C}(26)$ & $109.73(16)$ \\
\hline$C(5)-C(6)-C(1)$ & $121.87(18)$ & $\mathrm{C}(28)-\mathrm{C}(25)-\mathrm{C}(27)$ & $112.27(18)$ \\
\hline $\mathrm{C}(5)-\mathrm{C}(6)-\mathrm{H}(6)$ & 119.1 & $\mathrm{C}(26)-\mathrm{C}(25)-\mathrm{C}(27)$ & $110.33(17)$ \\
\hline $\mathrm{C}(1)-\mathrm{C}(6)-\mathrm{H}(6)$ & 119.1 & $C(28)-C(25)-S(1)$ & $103.88(13)$ \\
\hline $\mathrm{N}(1)-\mathrm{C}(7)-\mathrm{C}(12)$ & $121.09(15)$ & $C(26)-C(25)-S(1)$ & $110.75(13)$ \\
\hline $\mathrm{N}(1)-\mathrm{C}(7)-\mathrm{C}(8)$ & $121.46(15)$ & $\mathrm{C}(27)-\mathrm{C}(25)-\mathrm{S}(1)$ & $109.72(13)$ \\
\hline$C(12)-C(7)-C(8)$ & $117.17(16)$ & $\mathrm{C}(25)-\mathrm{C}(26)-\mathrm{H}(26 \mathrm{~A})$ & 109.5 \\
\hline$C(9)-C(8)-C(7)$ & 119.94(16) & $\mathrm{C}(25)-\mathrm{C}(26)-\mathrm{H}(26 \mathrm{~B})$ & 109.5 \\
\hline $\mathrm{C}(9)-\mathrm{C}(8)-\mathrm{S}(2)$ & $121.01(13)$ & $\mathrm{H}(26 \mathrm{~A})-\mathrm{C}(26)-\mathrm{H}(26 \mathrm{~B})$ & 109.5 \\
\hline $\mathrm{C}(7)-\mathrm{C}(8)-\mathrm{S}(2)$ & $118.96(13)$ & $\mathrm{C}(25)-\mathrm{C}(26)-\mathrm{H}(26 \mathrm{C})$ & 109.5 \\
\hline$C(10)-C(9)-C(8)$ & $121.60(16)$ & $\mathrm{H}(26 \mathrm{~A})-\mathrm{C}(26)-\mathrm{H}(26 \mathrm{C})$ & 109.5 \\
\hline
\end{tabular}




\begin{tabular}{|c|c|c|c|}
\hline $\mathrm{H}(26 \mathrm{~B})-\mathrm{C}(26)-\mathrm{H}(26 \mathrm{C})$ & 109.5 & $\mathrm{C}(33)-\mathrm{C}(34)-\mathrm{H}(34 \mathrm{~A})$ & 109.5 \\
\hline $\mathrm{C}(25)-\mathrm{C}(27)-\mathrm{H}(27 \mathrm{~A})$ & 109.5 & $\mathrm{C}(33)-\mathrm{C}(34)-\mathrm{H}(34 \mathrm{~B})$ & 109.5 \\
\hline $\mathrm{C}(25)-\mathrm{C}(27)-\mathrm{H}(27 \mathrm{~B})$ & 109.5 & $\mathrm{H}(34 \mathrm{~A})-\mathrm{C}(34)-\mathrm{H}(34 \mathrm{~B})$ & 109.5 \\
\hline $\mathrm{H}(27 \mathrm{~A})-\mathrm{C}(27)-\mathrm{H}(27 \mathrm{~B})$ & 109.5 & $\mathrm{C}(33)-\mathrm{C}(34)-\mathrm{H}(34 \mathrm{C})$ & 109.5 \\
\hline $\mathrm{C}(25)-\mathrm{C}(27)-\mathrm{H}(27 \mathrm{C})$ & 109.5 & $\mathrm{H}(34 \mathrm{~A})-\mathrm{C}(34)-\mathrm{H}(34 \mathrm{C})$ & 109.5 \\
\hline $\mathrm{H}(27 \mathrm{~A})-\mathrm{C}(27)-\mathrm{H}(27 \mathrm{C})$ & 109.5 & $\mathrm{H}(34 \mathrm{~B})-\mathrm{C}(34)-\mathrm{H}(34 \mathrm{C})$ & 109.5 \\
\hline $\mathrm{H}(27 \mathrm{~B})-\mathrm{C}(27)-\mathrm{H}(27 \mathrm{C})$ & 109.5 & $\mathrm{C}(33)-\mathrm{C}(35)-\mathrm{H}(35 \mathrm{~A})$ & 109.5 \\
\hline $\mathrm{C}(25)-\mathrm{C}(28)-\mathrm{H}(28 \mathrm{~A})$ & 109.5 & $\mathrm{C}(33)-\mathrm{C}(35)-\mathrm{H}(35 \mathrm{~B})$ & 109.5 \\
\hline $\mathrm{C}(25)-\mathrm{C}(28)-\mathrm{H}(28 \mathrm{~B})$ & 109.5 & $\mathrm{H}(35 \mathrm{~A})-\mathrm{C}(35)-\mathrm{H}(35 \mathrm{~B})$ & 109.5 \\
\hline $\mathrm{H}(28 \mathrm{~A})-\mathrm{C}(28)-\mathrm{H}(28 \mathrm{~B})$ & 109.5 & $\mathrm{C}(33)-\mathrm{C}(35)-\mathrm{H}(35 \mathrm{C})$ & 109.5 \\
\hline $\mathrm{C}(25)-\mathrm{C}(28)-\mathrm{H}(28 \mathrm{C})$ & 109.5 & $\mathrm{H}(35 \mathrm{~A})-\mathrm{C}(35)-\mathrm{H}(35 \mathrm{C})$ & 109.5 \\
\hline $\mathrm{H}(28 \mathrm{~A})-\mathrm{C}(28)-\mathrm{H}(28 \mathrm{C})$ & 109.5 & $\mathrm{H}(35 \mathrm{~B})-\mathrm{C}(35)-\mathrm{H}(35 \mathrm{C})$ & 109.5 \\
\hline $\mathrm{H}(28 \mathrm{~B})-\mathrm{C}(28)-\mathrm{H}(28 \mathrm{C})$ & 109.5 & $\mathrm{C}(33)-\mathrm{C}(36)-\mathrm{H}(36 \mathrm{~A})$ & 109.5 \\
\hline$C(32)-C(29)-C(30)$ & $112.65(17)$ & $\mathrm{C}(33)-\mathrm{C}(36)-\mathrm{H}(36 \mathrm{~B})$ & 109.5 \\
\hline $\mathrm{C}(32)-\mathrm{C}(29)-\mathrm{C}(31)$ & $109.43(16)$ & $\mathrm{H}(36 \mathrm{~A})-\mathrm{C}(36)-\mathrm{H}(36 \mathrm{~B})$ & 109.5 \\
\hline $\mathrm{C}(30)-\mathrm{C}(29)-\mathrm{C}(31)$ & $110.47(17)$ & $\mathrm{C}(33)-\mathrm{C}(36)-\mathrm{H}(36 \mathrm{C})$ & 109.5 \\
\hline$C(32)-C(29)-S(2)$ & $103.49(13)$ & $\mathrm{H}(36 \mathrm{~A})-\mathrm{C}(36)-\mathrm{H}(36 \mathrm{C})$ & 109.5 \\
\hline$C(30)-C(29)-S(2)$ & $109.87(13)$ & $\mathrm{H}(36 \mathrm{~B})-\mathrm{C}(36)-\mathrm{H}(36 \mathrm{C})$ & 109.5 \\
\hline$C(31)-C(29)-S(2)$ & $110.74(13)$ & $\mathrm{C}(39)-\mathrm{C}(37)-\mathrm{C}(38)$ & $111.35(16)$ \\
\hline $\mathrm{C}(29)-\mathrm{C}(30)-\mathrm{H}(30 \mathrm{~A})$ & 109.5 & $\mathrm{C}(39)-\mathrm{C}(37)-\mathrm{C}(40)$ & $110.15(16)$ \\
\hline $\mathrm{C}(29)-\mathrm{C}(30)-\mathrm{H}(30 \mathrm{~B})$ & 109.5 & $\mathrm{C}(38)-\mathrm{C}(37)-\mathrm{C}(40)$ & $110.57(15)$ \\
\hline $\mathrm{H}(30 \mathrm{~A})-\mathrm{C}(30)-\mathrm{H}(30 \mathrm{~B})$ & 109.5 & $\mathrm{C}(39)-\mathrm{C}(37)-\mathrm{S}(4)$ & $110.11(12)$ \\
\hline C(29)-C(30)-H(30C) & 109.5 & $\mathrm{C}(38)-\mathrm{C}(37)-\mathrm{S}(4)$ & $103.29(12)$ \\
\hline $\mathrm{H}(30 \mathrm{~A})-\mathrm{C}(30)-\mathrm{H}(30 \mathrm{C})$ & 109.5 & $\mathrm{C}(40)-\mathrm{C}(37)-\mathrm{S}(4)$ & $111.20(13)$ \\
\hline $\mathrm{H}(30 \mathrm{~B})-\mathrm{C}(30)-\mathrm{H}(30 \mathrm{C})$ & 109.5 & $\mathrm{C}(37)-\mathrm{C}(38)-\mathrm{H}(38 \mathrm{~A})$ & 109.5 \\
\hline $\mathrm{C}(29)-\mathrm{C}(31)-\mathrm{H}(31 \mathrm{~A})$ & 109.5 & $\mathrm{C}(37)-\mathrm{C}(38)-\mathrm{H}(38 \mathrm{~B})$ & 109.5 \\
\hline $\mathrm{C}(29)-\mathrm{C}(31)-\mathrm{H}(31 \mathrm{~B})$ & 109.5 & $\mathrm{H}(38 \mathrm{~A})-\mathrm{C}(38)-\mathrm{H}(38 \mathrm{~B})$ & 109.5 \\
\hline $\mathrm{H}(31 \mathrm{~A})-\mathrm{C}(31)-\mathrm{H}(31 \mathrm{~B})$ & 109.5 & $\mathrm{C}(37)-\mathrm{C}(38)-\mathrm{H}(38 \mathrm{C})$ & 109.5 \\
\hline $\mathrm{C}(29)-\mathrm{C}(31)-\mathrm{H}(31 \mathrm{C})$ & 109.5 & $\mathrm{H}(38 \mathrm{~A})-\mathrm{C}(38)-\mathrm{H}(38 \mathrm{C})$ & 109.5 \\
\hline $\mathrm{H}(31 \mathrm{~A})-\mathrm{C}(31)-\mathrm{H}(31 \mathrm{C})$ & 109.5 & $\mathrm{H}(38 \mathrm{~B})-\mathrm{C}(38)-\mathrm{H}(38 \mathrm{C})$ & 109.5 \\
\hline $\mathrm{H}(31 \mathrm{~B})-\mathrm{C}(31)-\mathrm{H}(31 \mathrm{C})$ & 109.5 & $\mathrm{C}(37)-\mathrm{C}(39)-\mathrm{H}(39 \mathrm{~A})$ & 109.5 \\
\hline $\mathrm{C}(29)-\mathrm{C}(32)-\mathrm{H}(32 \mathrm{~A})$ & 109.5 & $\mathrm{C}(37)-\mathrm{C}(39)-\mathrm{H}(39 \mathrm{~B})$ & 109.5 \\
\hline $\mathrm{C}(29)-\mathrm{C}(32)-\mathrm{H}(32 \mathrm{~B})$ & 109.5 & $\mathrm{H}(39 \mathrm{~A})-\mathrm{C}(39)-\mathrm{H}(39 \mathrm{~B})$ & 109.5 \\
\hline $\mathrm{H}(32 \mathrm{~A})-\mathrm{C}(32)-\mathrm{H}(32 \mathrm{~B})$ & 109.5 & $\mathrm{C}(37)-\mathrm{C}(39)-\mathrm{H}(39 \mathrm{C})$ & 109.5 \\
\hline $\mathrm{C}(29)-\mathrm{C}(32)-\mathrm{H}(32 \mathrm{C})$ & 109.5 & $\mathrm{H}(39 \mathrm{~A})-\mathrm{C}(39)-\mathrm{H}(39 \mathrm{C})$ & 109.5 \\
\hline $\mathrm{H}(32 \mathrm{~A})-\mathrm{C}(32)-\mathrm{H}(32 \mathrm{C})$ & 109.5 & $\mathrm{H}(39 \mathrm{~B})-\mathrm{C}(39)-\mathrm{H}(39 \mathrm{C})$ & 109.5 \\
\hline $\mathrm{H}(32 \mathrm{~B})-\mathrm{C}(32)-\mathrm{H}(32 \mathrm{C})$ & 109.5 & $\mathrm{C}(37)-\mathrm{C}(40)-\mathrm{H}(40 \mathrm{~A})$ & 109.5 \\
\hline$C(35)-C(33)-C(36)$ & $110.51(16)$ & $\mathrm{C}(37)-\mathrm{C}(40)-\mathrm{H}(40 \mathrm{~B})$ & 109.5 \\
\hline $\mathrm{C}(35)-\mathrm{C}(33)-\mathrm{C}(34)$ & $111.45(16)$ & $\mathrm{H}(40 \mathrm{~A})-\mathrm{C}(40)-\mathrm{H}(40 \mathrm{~B})$ & 109.5 \\
\hline $\mathrm{C}(36)-\mathrm{C}(33)-\mathrm{C}(34)$ & $109.90(16)$ & $\mathrm{C}(37)-\mathrm{C}(40)-\mathrm{H}(40 \mathrm{C})$ & 109.5 \\
\hline $\mathrm{C}(35)-\mathrm{C}(33)-\mathrm{S}(3)$ & $103.43(13)$ & $\mathrm{H}(40 \mathrm{~A})-\mathrm{C}(40)-\mathrm{H}(40 \mathrm{C})$ & 109.5 \\
\hline$C(36)-C(33)-S(3)$ & $111.30(13)$ & $\mathrm{H}(40 \mathrm{~B})-\mathrm{C}(40)-\mathrm{H}(40 \mathrm{C})$ & 109.5 \\
\hline $\mathrm{C}(34)-\mathrm{C}(33)-\mathrm{S}(3)$ & $110.12(13)$ & & \\
\hline
\end{tabular}


Table 4. Anisotropic displacement parameters $\left(\AA^{2} \times 10^{3}\right)$ for 2 . The anisotropic displacement factor exponent takes the form: $-2 \pi^{2}\left[h^{2} a^{* 2} U^{11}+\ldots+2 h k a^{*} b^{*} U^{12}\right]$

\begin{tabular}{|c|c|c|c|c|c|c|}
\hline & $\mathrm{U}^{11}$ & $\mathrm{U}^{22}$ & $\mathrm{U}^{33}$ & $\mathrm{U}^{23}$ & $\mathrm{U}^{13}$ & $\mathrm{U}^{12}$ \\
\hline$\overline{\mathrm{Cu}}(1)$ & $14(1)$ & $15(1)$ & $15(1)$ & 1(1) & $0(1)$ & $4(1)$ \\
\hline $\mathrm{Cu}(2)$ & $13(1)$ & $15(1)$ & $15(1)$ & $0(1)$ & 1(1) & $4(1)$ \\
\hline $\mathrm{N}(1)$ & $17(1)$ & $18(1)$ & $13(1)$ & $-1(1)$ & $-2(1)$ & $9(1)$ \\
\hline $\mathrm{N}(2)$ & $14(1)$ & $16(1)$ & $13(1)$ & 1(1) & $2(1)$ & $6(1)$ \\
\hline $\mathrm{S}(1)$ & $14(1)$ & $19(1)$ & $14(1)$ & $-3(1)$ & $0(1)$ & $4(1)$ \\
\hline$S(2)$ & $17(1)$ & $16(1)$ & $15(1)$ & $-1(1)$ & $-3(1)$ & $4(1)$ \\
\hline$S(3)$ & $13(1)$ & $23(1)$ & $17(1)$ & $7(1)$ & $1(1)$ & $4(1)$ \\
\hline S(4) & $17(1)$ & $14(1)$ & $16(1)$ & 1(1) & $5(1)$ & $5(1)$ \\
\hline $\mathrm{C}(1)$ & $13(1)$ & $22(1)$ & $16(1)$ & 1(1) & $0(1)$ & $7(1)$ \\
\hline$C(2)$ & $12(1)$ & $21(1)$ & $17(1)$ & $0(1)$ & $0(1)$ & $4(1)$ \\
\hline$C(3)$ & $18(1)$ & $30(1)$ & $15(1)$ & $1(1)$ & 1(1) & $4(1)$ \\
\hline$C(4)$ & $25(1)$ & $33(1)$ & $20(1)$ & $10(1)$ & $-1(1)$ & $9(1)$ \\
\hline$C(5)$ & $29(1)$ & $25(1)$ & $28(1)$ & $6(1)$ & $-3(1)$ & $12(1)$ \\
\hline$C(6)$ & $28(1)$ & $23(1)$ & $19(1)$ & $-1(1)$ & $-2(1)$ & $13(1)$ \\
\hline$C(7)$ & $21(1)$ & $14(1)$ & $14(1)$ & $0(1)$ & 1(1) & $7(1)$ \\
\hline $\mathrm{C}(8)$ & $18(1)$ & $13(1)$ & $18(1)$ & 1(1) & $-1(1)$ & $6(1)$ \\
\hline $\mathrm{C}(9)$ & $28(1)$ & $16(1)$ & $13(1)$ & $0(1)$ & $-1(1)$ & $6(1)$ \\
\hline $\mathrm{C}(10)$ & $29(1)$ & 21(1) & $17(1)$ & $0(1)$ & $7(1)$ & $9(1)$ \\
\hline $\mathrm{C}(11)$ & $23(1)$ & $27(1)$ & $25(1)$ & $-1(1)$ & $3(1)$ & $12(1)$ \\
\hline $\mathrm{C}(12)$ & $23(1)$ & $26(1)$ & $18(1)$ & $-3(1)$ & $-3(1)$ & $13(1)$ \\
\hline $\mathrm{C}(13)$ & $9(1)$ & $23(1)$ & $15(1)$ & $-2(1)$ & $-2(1)$ & $4(1)$ \\
\hline$C(14)$ & $11(1)$ & $25(1)$ & $16(1)$ & $0(1)$ & $-1(1)$ & $4(1)$ \\
\hline $\mathrm{C}(15)$ & $15(1)$ & $37(1)$ & $15(1)$ & $2(1)$ & $0(1)$ & $3(1)$ \\
\hline $\mathrm{C}(16)$ & $19(1)$ & $42(1)$ & $17(1)$ & $-9(1)$ & $2(1)$ & $4(1)$ \\
\hline $\mathrm{C}(17)$ & $17(1)$ & $30(1)$ & $28(1)$ & $-12(1)$ & 1(1) & $6(1)$ \\
\hline $\mathrm{C}(18)$ & $15(1)$ & $23(1)$ & $22(1)$ & $-2(1)$ & 1(1) & $4(1)$ \\
\hline $\mathrm{C}(19)$ & $18(1)$ & $13(1)$ & $16(1)$ & $0(1)$ & $2(1)$ & $5(1)$ \\
\hline $\mathrm{C}(20)$ & $20(1)$ & 11(1) & $16(1)$ & $-1(1)$ & $0(1)$ & $6(1)$ \\
\hline $\mathrm{C}(21)$ & $18(1)$ & $19(1)$ & $22(1)$ & 1(1) & 1(1) & $6(1)$ \\
\hline$C(22)$ & $22(1)$ & $20(1)$ & $27(1)$ & $1(1)$ & $-6(1)$ & $9(1)$ \\
\hline $\mathrm{C}(23)$ & $32(1)$ & $18(1)$ & $19(1)$ & 1(1) & $-8(1)$ & $6(1)$ \\
\hline$C(24)$ & $27(1)$ & $15(1)$ & $16(1)$ & 1(1) & 1(1) & $3(1)$ \\
\hline$C(25)$ & $14(1)$ & $30(1)$ & $22(1)$ & $-3(1)$ & $-5(1)$ & $-1(1)$ \\
\hline$C(26)$ & $24(1)$ & $40(1)$ & $24(1)$ & $-1(1)$ & $-9(1)$ & $-1(1)$ \\
\hline $\mathrm{C}(27)$ & $15(1)$ & $54(2)$ & $30(1)$ & $-4(1)$ & $1(1)$ & $5(1)$ \\
\hline $\mathrm{C}(28)$ & $32(1)$ & $28(1)$ & $30(1)$ & $-2(1)$ & $-7(1)$ & $-7(1)$ \\
\hline C(29) & $27(1)$ & $17(1)$ & $22(1)$ & $-6(1)$ & $-1(1)$ & $-2(1)$ \\
\hline$C(30)$ & $43(1)$ & $17(1)$ & $33(1)$ & $0(1)$ & $3(1)$ & $3(1)$ \\
\hline $\mathrm{C}(31)$ & $37(1)$ & $27(1)$ & $27(1)$ & $-11(1)$ & $2(1)$ & $-2(1)$ \\
\hline $\mathrm{C}(32)$ & $26(1)$ & $34(1)$ & $28(1)$ & $-7(1)$ & $-2(1)$ & $-6(1)$ \\
\hline$C(33)$ & $13(1)$ & $28(1)$ & $22(1)$ & $9(1)$ & 1(1) & $0(1)$ \\
\hline$C(34)$ & $18(1)$ & $31(1)$ & $26(1)$ & $7(1)$ & $-5(1)$ & $3(1)$ \\
\hline$C(35)$ & $27(1)$ & $26(1)$ & $37(1)$ & $12(1)$ & $-1(1)$ & $-1(1)$ \\
\hline$C(36)$ & $19(1)$ & $48(1)$ & $26(1)$ & 12(1) & $5(1)$ & $0(1)$ \\
\hline $\mathrm{C}(37)$ & $23(1)$ & $12(1)$ & $23(1)$ & 1(1) & $7(1)$ & $2(1)$ \\
\hline C(38) & $22(1)$ & $25(1)$ & $29(1)$ & 1(1) & $6(1)$ & $-1(1)$ \\
\hline C(39) & $30(1)$ & $17(1)$ & $28(1)$ & $-4(1)$ & $4(1)$ & $6(1)$ \\
\hline $\mathrm{C}(40)$ & $35(1)$ & $16(1)$ & $31(1)$ & $8(1)$ & $9(1)$ & $4(1)$ \\
\hline
\end{tabular}


Table 5. Hydrogen coordinates ( $\left.\times 10^{4}\right)$ and isotropic displacement parameters $\left(\AA^{2} \times 10^{3}\right)$ for 2 .

\begin{tabular}{|c|c|c|c|c|}
\hline & $\mathrm{x}$ & $\mathrm{y}$ & $\mathrm{z}$ & $\mathrm{U}_{\mathrm{eq}}$ \\
\hline $\mathrm{H}(3)$ & 6029 & 439 & 4714 & 26 \\
\hline $\mathrm{H}(4)$ & 5861 & -1790 & 4733 & 31 \\
\hline $\mathrm{H}(5)$ & 5604 & -3106 & 3693 & 32 \\
\hline $\mathrm{H}(6)$ & 5415 & -2213 & 2656 & 27 \\
\hline $\mathrm{H}(9)$ & 5151 & -969 & 176 & 23 \\
\hline $\mathrm{H}(10)$ & 7459 & -813 & 161 & 26 \\
\hline $\mathrm{H}(11)$ & 8769 & -324 & 1201 & 29 \\
\hline $\mathrm{H}(12)$ & 7761 & 67 & 2221 & 26 \\
\hline $\mathrm{H}(15)$ & 1853 & 1153 & 4631 & 29 \\
\hline $\mathrm{H}(16)$ & 1874 & 3319 & 4931 & 33 \\
\hline $\mathrm{H}(17)$ & 2328 & 4857 & 4095 & 31 \\
\hline $\mathrm{H}(18)$ & 2811 & 4259 & 2984 & 25 \\
\hline $\mathrm{H}(21)$ & 767 & 2327 & 2139 & 23 \\
\hline $\mathrm{H}(22)$ & 63 & 2862 & 1096 & 27 \\
\hline $\mathrm{H}(23)$ & 1595 & 3360 & 198 & 28 \\
\hline $\mathrm{H}(24)$ & 3855 & 3376 & 374 & 24 \\
\hline $\mathrm{H}(26 \mathrm{~A})$ & 9074 & 3394 & 4732 & 48 \\
\hline $\mathrm{H}(26 \mathrm{~B})$ & 8150 & 1883 & 4617 & 48 \\
\hline $\mathrm{H}(26 \mathrm{C})$ & 7496 & 2985 & 4930 & 48 \\
\hline $\mathrm{H}(27 \mathrm{~A})$ & 8376 & 2743 & 2889 & 52 \\
\hline $\mathrm{H}(27 \mathrm{~B})$ & 8510 & 1635 & 3378 & 52 \\
\hline $\mathrm{H}(27 \mathrm{C})$ & 9608 & 3074 & 3451 & 52 \\
\hline $\mathrm{H}(28 \mathrm{~A})$ & 7230 & 4727 & 4169 & 50 \\
\hline $\mathrm{H}(28 \mathrm{~B})$ & 7609 & 4641 & 3376 & 50 \\
\hline $\mathrm{H}(28 \mathrm{C})$ & 8798 & 5035 & 3963 & 50 \\
\hline $\mathrm{H}(30 \mathrm{~A})$ & 2630 & -2978 & 1988 & 48 \\
\hline $\mathrm{H}(30 \mathrm{~B})$ & 3783 & -3165 & 1476 & 48 \\
\hline $\mathrm{H}(30 \mathrm{C})$ & 2283 & -4209 & 1423 & 48 \\
\hline $\mathrm{H}(31 \mathrm{~A})$ & 2015 & -3795 & 152 & 50 \\
\hline $\mathrm{H}(31 \mathrm{~B})$ & 3574 & -2884 & 240 & 50 \\
\hline $\mathrm{H}(31 \mathrm{C})$ & 2423 & -2293 & -45 & 50 \\
\hline $\mathrm{H}(32 \mathrm{~A})$ & 279 & -3433 & 944 & 49 \\
\hline $\mathrm{H}(32 \mathrm{~B})$ & 633 & -1932 & 750 & 49 \\
\hline $\mathrm{H}(32 \mathrm{C})$ & 683 & -2252 & 1538 & 49 \\
\hline $\mathrm{H}(34 \mathrm{~A})$ & 135 & -836 & 2367 & 39 \\
\hline $\mathrm{H}(34 \mathrm{~B})$ & -223 & 171 & 2930 & 39 \\
\hline $\mathrm{H}(34 \mathrm{C})$ & -1250 & -1292 & 2780 & 39 \\
\hline $\mathrm{H}(35 \mathrm{~A})$ & -448 & -3240 & 3126 & 47 \\
\hline $\mathrm{H}(35 \mathrm{~B})$ & 990 & -2929 & 3537 & 47 \\
\hline $\mathrm{H}(35 \mathrm{C})$ & 955 & -2677 & 2738 & 47 \\
\hline $\mathrm{H}(36 \mathrm{~A})$ & -1152 & -1736 & 4020 & 49 \\
\hline $\mathrm{H}(36 \mathrm{~B})$ & -190 & -247 & 4173 & 49 \\
\hline $\mathrm{H}(36 \mathrm{C})$ & 305 & -1449 & 4409 & 49 \\
\hline $\mathrm{H}(38 \mathrm{~A})$ & 8446 & 5683 & 1813 & 40 \\
\hline $\mathrm{H}(38 \mathrm{~B})$ & 8165 & 4368 & 1309 & 40 \\
\hline $\mathrm{H}(38 \mathrm{C})$ & 7934 & 4262 & 2116 & 40 \\
\hline $\mathrm{H}(39 \mathrm{~A})$ & 5956 & 4905 & 2643 & 39 \\
\hline H(39B) & 4915 & 5334 & 2159 & 39 \\
\hline $\mathrm{H}(39 \mathrm{C})$ & 6431 & 6307 & 2319 & 39 \\
\hline $\mathrm{H}(40 \mathrm{~A})$ & 5266 & 5431 & 912 & 41 \\
\hline $\mathrm{H}(40 \mathrm{~B})$ & 6502 & 5006 & 570 & 41 \\
\hline $\mathrm{H}(40 \mathrm{C})$ & 6807 & 6374 & 1032 & 41 \\
\hline
\end{tabular}


Table 6. Crystal data and structure refinement for 3 .

\begin{tabular}{|c|c|c|}
\hline Name of Complex & \multicolumn{2}{|c|}{$[(\mathrm{SNS}) \mathrm{Cu}]_{2}\left[\mathrm{~B}\left(\mathrm{C}_{6} \mathrm{H}_{3}\left(\mathrm{CF}_{3}\right)_{2}\right)_{4}\right]$} \\
\hline Empirical formula & \multicolumn{2}{|c|}{$\mathrm{C}_{72} \mathrm{H}_{64} \mathrm{BCu}_{2} \mathrm{~F}_{24} \mathrm{~N}_{2} \mathrm{~S}_{4} \cdot 1 / 2\left(\mathrm{C}_{4} \mathrm{H}_{10} \mathrm{O}\right)$} \\
\hline Formula weight & \multicolumn{2}{|l|}{1716.478} \\
\hline Temperature & \multicolumn{2}{|l|}{$100 \mathrm{~K}$} \\
\hline Wavelength & \multicolumn{2}{|l|}{$0.71073 \AA$} \\
\hline Crystal system & \multicolumn{2}{|l|}{ Triclinic } \\
\hline Crystal habit & \multicolumn{2}{|l|}{ blade } \\
\hline Crystal color & \multicolumn{2}{|l|}{ deep red } \\
\hline Crystal size & \multicolumn{2}{|c|}{$0.34 \times 0.28 \times 0.14 \mathrm{~mm}^{3}$} \\
\hline Space group & \multicolumn{2}{|l|}{ P-1 (\#2) } \\
\hline \multirow[t]{3}{*}{ Unit cell dimensions } & $\mathrm{a}=10.3179(8) \AA$ & $\alpha=74.234(1)^{\circ}$ \\
\hline & $\mathrm{b}=19.3779(14) \AA$ & $\beta=83.222(1)^{\circ}$ \\
\hline & $\mathrm{c}=19.9116(15) \AA$ & $\gamma=85.603(1)^{\circ}$ \\
\hline Volume & \multicolumn{2}{|l|}{$3800.4(5) \AA^{3}$} \\
\hline $\mathrm{Z}$ & \multicolumn{2}{|l|}{2} \\
\hline Density (calculated) & \multicolumn{2}{|l|}{$1.500 \mathrm{~g} / \mathrm{cm}^{3}$} \\
\hline Absorption coefficient & \multicolumn{2}{|l|}{$0.773 \mathrm{~mm}^{-1}$} \\
\hline $\mathrm{F}(000)$ & \multicolumn{2}{|l|}{1748} \\
\hline Theta range for data collection & \multicolumn{2}{|l|}{1.72 to $28.48^{\circ}$. } \\
\hline Index ranges & \multicolumn{2}{|c|}{$-13<=\mathrm{h}<=13,-25<=\mathrm{k}<=25,-26<=1<=26$} \\
\hline Reflections collected & \multicolumn{2}{|l|}{79590} \\
\hline Independent reflections & \multicolumn{2}{|c|}{$17675[\mathrm{R}(\mathrm{int})=0.0552]$} \\
\hline Completeness to theta $=28.48^{\circ}$ & \multicolumn{2}{|l|}{$91.9 \%$} \\
\hline Absorption correction & \multicolumn{2}{|l|}{ None } \\
\hline Refinement method & \multicolumn{2}{|c|}{ Full-matrix least-squares on $\mathrm{F}^{2}$} \\
\hline Data / restraints / parameters & \multicolumn{2}{|l|}{$17675 / 0 / 1004$} \\
\hline Goodness-of-fit on $\mathrm{F}^{2}$ & \multicolumn{2}{|l|}{1.580} \\
\hline Final $R$ indices $[\mathrm{I}>2 \operatorname{sigma}(\mathrm{I})]$ & \multicolumn{2}{|c|}{$\mathrm{R} 1=0.0393, \mathrm{wR} 2=0.0810$} \\
\hline $\mathrm{R}$ indices (all data) & \multicolumn{2}{|c|}{$\mathrm{R} 1=0.0597, \mathrm{wR} 2=0.0850$} \\
\hline Largest diff. peak and hole & \multicolumn{2}{|l|}{0.61 and -0.50 e. $\AA^{-3}$} \\
\hline
\end{tabular}

\section{Special Refinement Details}

Refinement of F2 against ALL reflections. The weighted R-factor $(w \mathrm{R})$ and goodness of fit (S) are based on F2, conventional R-factors (R) are based on F, with F set to zero for negative F2. The threshold expression of F2 $>2$ ( (F2) is used only for calculating R-factors(gt) etc. and is not relevant to the choice of reflections for refinement.

All esds (except the esd in the dihedral angle between two 1.s. planes) are estimated using the full covariance matrix. The cell esds are taken into account individually in the estimation of esds in distances, angles and torsion angles; correlations between esds in cell parameters are only used when they are defined by crystal symmetry. An approximate (isotropic) treatment of cell esds is used for estimating esds involving l.s. planes.

One $\mathrm{CF}_{3}$ group is disordered and was modeled over 2 locations with one relative population parameter, $\{\mathrm{F} 4, \mathrm{~F} 5$, and F6 $\}$ and $\{\mathrm{F} 4 \mathrm{~A}, \mathrm{~F} 5 \mathrm{~A}$, and F6A; isotropically $\}$ in $92.7(4) \%$ and $7.3(4) \%$ respectively. Additionally $1 / 2$ molecule of diethyl ether is contained in the asymmetric unit with the oxygen atom placed on a center of symmetry $(1.0,0.5,0.5)$ and the alpha carbon split over 2 locations. 
Table 7. Atomic coordinates ( $\left.\mathrm{x} 10^{4}\right)$ and equivalent isotropic displacement parameters $\left(\AA^{2} \times 10^{3}\right)$ for 3. $U_{\text {eq }}$ is defined as one third of the trace of the orthogonalized $U^{i j}$ tensor.

\begin{tabular}{|c|c|c|c|c|}
\hline & $\mathrm{x}$ & $\mathrm{y}$ & $\mathrm{Z}$ & $\mathrm{U}_{\mathrm{eq}}$ \\
\hline $\mathrm{Cu}(1)$ & $8093(1)$ & $8303(1)$ & $7388(1)$ & $17(1)$ \\
\hline $\mathrm{Cu}(2)$ & $6977(1)$ & $7654(1)$ & $6728(1)$ & $18(1)$ \\
\hline $\mathrm{N}(1)$ & $6092(2)$ & $8339(1)$ & $7295(1)$ & $17(1)$ \\
\hline $\mathrm{N}(2)$ & $8966(2)$ & $7668(1)$ & $6738(1)$ & $17(1)$ \\
\hline $\mathrm{S}(1)$ & $6133(1)$ & $6726(1)$ & $7604(1)$ & $22(1)$ \\
\hline $\mathrm{S}(2)$ & 8101(1) & $9476(1)$ & $6755(1)$ & $18(1)$ \\
\hline $\mathrm{S}(3)$ & $8704(1)$ & $7405(1)$ & $8316(1)$ & $22(1)$ \\
\hline S(4) & $7230(1)$ & $8278(1)$ & $5575(1)$ & 21(1) \\
\hline $\mathrm{O}$ & 10000 & 5000 & 5000 & $195(6)$ \\
\hline $\mathrm{B}(1)$ & $3879(2)$ & $7327(1)$ & 1943(1) & $16(1)$ \\
\hline $\mathrm{C}(1)$ & $5340(2)$ & $8007(1)$ & $7922(1)$ & $19(1)$ \\
\hline $\mathrm{C}(2)$ & $5395(2)$ & $7254(1)$ & $8168(1)$ & $22(1)$ \\
\hline $\mathrm{C}(3)$ & $4907(2)$ & $6926(1)$ & $8850(1)$ & $27(1)$ \\
\hline $\mathrm{C}(4)$ & $4281(2)$ & $7324(1)$ & $9280(1)$ & $30(1)$ \\
\hline$C(5)$ & $4130(2)$ & $8063(1)$ & $9023(1)$ & $25(1)$ \\
\hline $\mathrm{C}(6)$ & $4662(2)$ & $8403(1)$ & $8356(1)$ & 21(1) \\
\hline$C(7)$ & $5602(2)$ & $8995(1)$ & $6870(1)$ & $17(1)$ \\
\hline $\mathrm{C}(8)$ & $6463(2)$ & $9540(1)$ & $6529(1)$ & $18(1)$ \\
\hline $\mathrm{C}(9)$ & $6048(2)$ & $10119(1)$ & $6002(1)$ & $24(1)$ \\
\hline $\mathrm{C}(10)$ & $4777(2)$ & $10184(1)$ & $5828(1)$ & $27(1)$ \\
\hline $\mathrm{C}(11)$ & $3906(2)$ & 9671(1) & $6197(1)$ & $26(1)$ \\
\hline$C(12)$ & $4308(2)$ & $9086(1)$ & $6707(1)$ & $22(1)$ \\
\hline$C(13)$ & $9565(2)$ & $7011(1)$ & $7095(1)$ & $19(1)$ \\
\hline $\mathrm{C}(14)$ & $9361(2)$ & $6786(1)$ & $7832(1)$ & $22(1)$ \\
\hline$C(15)$ & $9671(2)$ & $6078(1)$ & $8182(1)$ & $28(1)$ \\
\hline$C(16)$ & $10267(2)$ & $5606(1)$ & $7821(1)$ & $33(1)$ \\
\hline$C(17)$ & $10568(2)$ & $5842(1)$ & $7101(1)$ & $30(1)$ \\
\hline $\mathrm{C}(18)$ & $10221(2)$ & $6536(1)$ & $6739(1)$ & $24(1)$ \\
\hline$C(19)$ & $9619(2)$ & $8084(1)$ & $6120(1)$ & $18(1)$ \\
\hline $\mathrm{C}(20)$ & $8884(2)$ & $8475(1)$ & $5573(1)$ & $20(1)$ \\
\hline $\mathrm{C}(21)$ & $9456(2)$ & $9015(1)$ & $5031(1)$ & $25(1)$ \\
\hline $\mathrm{C}(22)$ & $10757(2)$ & $9144(1)$ & 4998(1) & $27(1)$ \\
\hline $\mathrm{C}(23)$ & $11512(2)$ & $8724(1)$ & $5502(1)$ & $26(1)$ \\
\hline $\mathrm{C}(24)$ & $10953(2)$ & $8202(1)$ & $6055(1)$ & 21(1) \\
\hline$C(25)$ & $4710(2)$ & $6392(1)$ & $7303(1)$ & $30(1)$ \\
\hline$C(26)$ & $5348(2)$ & $5949(1)$ & $6818(2)$ & $44(1)$ \\
\hline $\mathrm{C}(27)$ & $3916(2)$ & 5921(1) & $7933(2)$ & $44(1)$ \\
\hline $\mathrm{C}(28)$ & $3870(2)$ & $7017(1)$ & $6917(1)$ & $30(1)$ \\
\hline C(29) & $8080(2)$ & $10094(1)$ & $7337(1)$ & $24(1)$ \\
\hline $\mathrm{C}(30)$ & $9481(2)$ & $10033(1)$ & $7517(1)$ & $35(1)$ \\
\hline $\mathrm{C}(31)$ & $7106(2)$ & $9858(1)$ & $7979(1)$ & $28(1)$ \\
\hline$C(32)$ & $7739(3)$ & $10859(1)$ & $6929(1)$ & $34(1)$ \\
\hline C(33) & $10200(2)$ & $7650(1)$ & $8637(1)$ & $26(1)$ \\
\hline$C(34)$ & $11171(2)$ & $7980(1)$ & 8013(1) & $30(1)$ \\
\hline$C(35)$ & $10814(2)$ & $6985(1)$ & $9115(1)$ & $36(1)$ \\
\hline$C(36)$ & $9680(2)$ & $8182(1)$ & $9049(1)$ & $34(1)$ \\
\hline$C(37)$ & $7366(2)$ & $7681(1)$ & $4965(1)$ & $29(1)$ \\
\hline $\mathrm{C}(38)$ & $7947(3)$ & $8093(1)$ & $4244(1)$ & $40(1)$ \\
\hline C(39) & $8177(2)$ & $7000(1)$ & $5249(1)$ & $31(1)$ \\
\hline $\mathrm{C}(40)$ & $5946(2)$ & $7528(2)$ & $4945(2)$ & $43(1)$ \\
\hline C(41) & $4344(2)$ & $6747(1)$ & $1485(1)$ & $17(1)$ \\
\hline$C(42)$ & $3483(2)$ & $6334(1)$ & $1286(1)$ & $18(1)$ \\
\hline C(43) & $3899(2)$ & $5848(1)$ & 899(1) & $20(1)$ \\
\hline C(44) & $5218(2)$ & $5750(1)$ & $689(1)$ & $22(1)$ \\
\hline
\end{tabular}




\begin{tabular}{|c|c|c|c|c|}
\hline $\mathrm{C}(45)$ & $6100(2)$ & $6150(1)$ & $877(1)$ & $21(1)$ \\
\hline$C(46)$ & $5672(2)$ & $6635(1)$ & $1264(1)$ & 19(1) \\
\hline C(47) & $2948(2)$ & $5435(1)$ & $674(1)$ & $26(1)$ \\
\hline C(48) & $7525(2)$ & $6058(1)$ & $652(1)$ & $29(1)$ \\
\hline C(49) & $4028(2)$ & $6950(1)$ & $2776(1)$ & $17(1)$ \\
\hline$C(50)$ & $3258(2)$ & 7185(1) & $3306(1)$ & 19(1) \\
\hline $\mathrm{C}(51)$ & $3404(2)$ & 6892(1) & 4014(1) & $22(1)$ \\
\hline $\mathrm{C}(52)$ & $4335(2)$ & $6352(1)$ & $4221(1)$ & 21(1) \\
\hline$C(53)$ & $5127(2)$ & $6114(1)$ & $3712(1)$ & 19(1) \\
\hline$C(54)$ & $4966(2)$ & $6400(1)$ & $3005(1)$ & $18(1)$ \\
\hline $\mathrm{C}(55)$ & $2573(2)$ & $7194(2)$ & $4542(1)$ & $36(1)$ \\
\hline $\mathrm{C}(56)$ & $6160(2)$ & $5542(1)$ & $3940(1)$ & $30(1)$ \\
\hline $\mathrm{C}(57)$ & $4826(2)$ & 8013(1) & $1663(1)$ & $16(1)$ \\
\hline C(58) & $5372(2)$ & $8338(1)$ & 2099(1) & $17(1)$ \\
\hline $\mathrm{C}(59)$ & $6171(2)$ & $8920(1)$ & $1837(1)$ & $18(1)$ \\
\hline $\mathrm{C}(60)$ & $6452(2)$ & $9209(1)$ & $1123(1)$ & $18(1)$ \\
\hline$C(61)$ & $5919(2)$ & $8897(1)$ & $677(1)$ & $17(1)$ \\
\hline$C(62)$ & 5133(2) & $8313(1)$ & $940(1)$ & $17(1)$ \\
\hline $\mathrm{C}(63)$ & $6782(2)$ & $9220(1)$ & $2334(1)$ & $24(1)$ \\
\hline$C(64)$ & 6191(2) & $9172(1)$ & $-101(1)$ & 21(1) \\
\hline$C(65)$ & $2352(2)$ & 7583(1) & $1829(1)$ & $18(1)$ \\
\hline$C(66)$ & $1323(2)$ & $7158(1)$ & $2205(1)$ & $20(1)$ \\
\hline $\mathrm{C}(67)$ & $29(2)$ & $7326(1)$ & $2075(1)$ & 21(1) \\
\hline $\mathrm{C}(68)$ & $-309(2)$ & $7925(1)$ & $1555(1)$ & $23(1)$ \\
\hline $\mathrm{C}(69)$ & $681(2)$ & $8359(1)$ & $1176(1)$ & $21(1)$ \\
\hline$C(70)$ & 1975(2) & 8194(1) & 1319(1) & $18(1)$ \\
\hline$C(71)$ & $-993(2)$ & $6835(1)$ & $2506(1)$ & $30(1)$ \\
\hline$C(72)$ & $370(2)$ & $9020(1)$ & $621(1)$ & $27(1)$ \\
\hline$C(73)^{c}$ & $9811(12)$ & $5069(7)$ & $5551(6)$ & $87(4)$ \\
\hline$C(74)^{c}$ & $8830(6)$ & $4557(3)$ & $5588(4)$ & $46(2)$ \\
\hline$C(75)$ & $8491(5)$ & $4747(2)$ & $6130(4)$ & $136(2)$ \\
\hline $\mathrm{F}(1)$ & $1715(1)$ & $5542(1)$ & $919(1)$ & $48(1)$ \\
\hline$F(2)$ & $3218(1)$ & $4727(1)$ & $864(1)$ & $42(1)$ \\
\hline$F(3)$ & $2963(1)$ & $5605(1)$ & $-29(1)$ & $40(1)$ \\
\hline $\mathrm{F}(4)^{\mathrm{a}}$ & $7986(2)$ & $5389(1)$ & $915(1)$ & $77(1)$ \\
\hline $\mathrm{F}(5)^{\mathrm{a}}$ & $7768(2)$ & $6144(1)$ & $-31(1)$ & $55(1)$ \\
\hline $\mathrm{F}(6)^{\mathrm{a}}$ & $8264(2)$ & $6488(2)$ & $815(2)$ & $93(1)$ \\
\hline$F(4 A)^{b}$ & $8204(17)$ & $5944(11)$ & $1150(9)$ & $22(5)$ \\
\hline$F(5 A)^{b}$ & $7929(18)$ & $6628(11)$ & $188(11)$ & $36(6)$ \\
\hline $\mathrm{F}(6 \mathrm{~A})^{\mathrm{b}}$ & $7899(19)$ & $5600(12)$ & 391(13) & $35(6)$ \\
\hline $\mathrm{F}(7)$ & $2960(2)$ & $7849(1)$ & $4539(1)$ & $56(1)$ \\
\hline $\mathrm{F}(8)$ & $1339(1)$ & $7319(1)$ & $4415(1)$ & $59(1)$ \\
\hline$F(9)$ & $2627(2)$ & $6805(1)$ & $5194(1)$ & $74(1)$ \\
\hline $\mathrm{F}(10)$ & $5653(2)$ & $4915(1)$ & $4292(1)$ & $49(1)$ \\
\hline$F(11)$ & $6901(2)$ & $5708(1)$ & $4369(1)$ & $66(1)$ \\
\hline$F(12)$ & 6950(1) & $5398(1)$ & $3411(1)$ & $41(1)$ \\
\hline $\mathrm{F}(13)$ & $6036(1)$ & 9164(1) & 2939(1) & $44(1)$ \\
\hline $\mathrm{F}(14)$ & $7919(1)$ & $8883(1)$ & $2509(1)$ & $39(1)$ \\
\hline$F(15)$ & $7034(2)$ & 9913(1) & 2074(1) & $49(1)$ \\
\hline$F(16)$ & $6930(1)$ & $9743(1)$ & $-297(1)$ & $33(1)$ \\
\hline $\mathrm{F}(17)$ & $6796(1)$ & $8671(1)$ & $-392(1)$ & $32(1)$ \\
\hline $\mathrm{F}(18)$ & $5087(1)$ & $9374(1)$ & $-419(1)$ & $26(1)$ \\
\hline$F(19)$ & $-811(2)$ & $6173(1)$ & $2434(1)$ & $63(1)$ \\
\hline$F(20)$ & $-998(2)$ & 6771(1) & $3187(1)$ & $55(1)$ \\
\hline $\mathrm{F}(21)$ & $-2192(1)$ & 7057(1) & $2346(1)$ & $61(1)$ \\
\hline$F(22)$ & $-733(1)$ & 8976(1) & $341(1)$ & $37(1)$ \\
\hline$F(23)$ & $207(2)$ & $9603(1)$ & $861(1)$ & $53(1)$ \\
\hline$F(24)$ & $1308(1)$ & $9162(1)$ & $82(1)$ & $38(1)$ \\
\hline
\end{tabular}


a: population: 0.927(4) / b: population: 0.073(4) / c: population: $1 / 2$ 
Table 8. Bond lengths $[\AA]$ and angles $\left[{ }^{\circ}\right]$ for 3 .

\begin{tabular}{|c|c|c|c|}
\hline$\overline{\mathrm{Cu}(1)-\mathrm{Cu}(2)}$ & $2.4724(4)$ & $\mathrm{C}(17)-\mathrm{C}(18)$ & $1.385(3)$ \\
\hline $\mathrm{Cu}(1)-\mathrm{N}(1)$ & $2.0887(16)$ & $\mathrm{C}(17)-\mathrm{H}(17)$ & 0.9500 \\
\hline $\mathrm{Cu}(1)-\mathrm{N}(2)$ & $2.1011(17)$ & $\mathrm{C}(18)-\mathrm{H}(18)$ & 0.9500 \\
\hline $\mathrm{Cu}(1)-\mathrm{S}(2)$ & $2.2805(6)$ & $C(19)-C(24)$ & $1.399(3)$ \\
\hline $\mathrm{Cu}(1)-\mathrm{S}(3)$ & $2.2805(6)$ & $C(19)-C(20)$ & $1.411(3)$ \\
\hline $\mathrm{Cu}(2)-\mathrm{N}(2)$ & $2.0568(16)$ & $\mathrm{C}(20)-\mathrm{C}(21)$ & $1.392(3)$ \\
\hline $\mathrm{Cu}(2)-\mathrm{N}(1)$ & $2.0641(16)$ & $\mathrm{C}(21)-\mathrm{C}(22)$ & $1.375(3)$ \\
\hline $\mathrm{Cu}(2)-\mathrm{S}(4)$ & $2.2797(6)$ & $\mathrm{C}(21)-\mathrm{H}(21)$ & 0.9500 \\
\hline $\mathrm{Cu}(2)-\mathrm{S}(1)$ & $2.2834(6)$ & $\mathrm{C}(22)-\mathrm{C}(23)$ & $1.385(3)$ \\
\hline $\mathrm{N}(1)-\mathrm{C}(1)$ & $1.409(2)$ & $\mathrm{C}(22)-\mathrm{H}(22)$ & 0.9500 \\
\hline $\mathrm{N}(1)-\mathrm{C}(7)$ & $1.416(2)$ & $C(23)-C(24)$ & $1.379(3)$ \\
\hline $\mathrm{N}(2)-\mathrm{C}(19)$ & $1.403(2)$ & $\mathrm{C}(23)-\mathrm{H}(23)$ & 0.9500 \\
\hline $\mathrm{N}(2)-\mathrm{C}(13)$ & $1.415(2)$ & $\mathrm{C}(24)-\mathrm{H}(24)$ & 0.9500 \\
\hline $\mathrm{S}(1)-\mathrm{C}(2)$ & $1.786(2)$ & $\mathrm{C}(25)-\mathrm{C}(28)$ & $1.517(3)$ \\
\hline $\mathrm{S}(1)-\mathrm{C}(25)$ & $1.870(2)$ & $C(25)-C(26)$ & $1.523(3)$ \\
\hline $\mathrm{S}(2)-\mathrm{C}(8)$ & $1.785(2)$ & $C(25)-C(27)$ & $1.526(3)$ \\
\hline$S(2)-C(29)$ & $1.877(2)$ & $\mathrm{C}(26)-\mathrm{H}(26 \mathrm{~A})$ & 0.9800 \\
\hline $\mathrm{S}(3)-\mathrm{C}(14)$ & $1.783(2)$ & $\mathrm{C}(26)-\mathrm{H}(26 \mathrm{~B})$ & 0.9800 \\
\hline $\mathrm{S}(3)-\mathrm{C}(33)$ & $1.878(2)$ & $\mathrm{C}(26)-\mathrm{H}(26 \mathrm{C})$ & 0.9800 \\
\hline$S(4)-C(20)$ & $1.777(2)$ & $\mathrm{C}(27)-\mathrm{H}(27 \mathrm{~A})$ & 0.9800 \\
\hline$S(4)-C(37)$ & $1.880(2)$ & $\mathrm{C}(27)-\mathrm{H}(27 \mathrm{~B})$ & 0.9800 \\
\hline $\mathrm{O}-\mathrm{C}(73)$ & $1.133(11)$ & $\mathrm{C}(27)-\mathrm{H}(27 \mathrm{C})$ & 0.9800 \\
\hline O-C(73)\#1 & $1.133(11)$ & $\mathrm{C}(28)-\mathrm{H}(28 \mathrm{~A})$ & 0.9800 \\
\hline $\mathrm{O}-\mathrm{C}(74)$ & $1.687(6)$ & $\mathrm{C}(28)-\mathrm{H}(28 \mathrm{~B})$ & 0.9800 \\
\hline $\mathrm{O}-\mathrm{C}(74) \# 1$ & $1.687(6)$ & $\mathrm{C}(28)-\mathrm{H}(28 \mathrm{C})$ & 0.9800 \\
\hline $\mathrm{B}(1)-\mathrm{C}(65)$ & $1.638(3)$ & $C(29)-C(31)$ & $1.518(3)$ \\
\hline $\mathrm{B}(1)-\mathrm{C}(49)$ & $1.641(3)$ & $C(29)-C(30)$ & $1.519(3)$ \\
\hline $\mathrm{B}(1)-\mathrm{C}(41)$ & $1.642(3)$ & $C(29)-C(32)$ & $1.522(3)$ \\
\hline $\mathrm{B}(1)-\mathrm{C}(57)$ & $1.643(3)$ & $\mathrm{C}(30)-\mathrm{H}(30 \mathrm{~A})$ & 0.9800 \\
\hline$C(1)-C(6)$ & $1.399(3)$ & $\mathrm{C}(30)-\mathrm{H}(30 \mathrm{~B})$ & 0.9800 \\
\hline$C(1)-C(2)$ & $1.406(3)$ & $\mathrm{C}(30)-\mathrm{H}(30 \mathrm{C})$ & 0.9800 \\
\hline $\mathrm{C}(2)-\mathrm{C}(3)$ & $1.386(3)$ & $\mathrm{C}(31)-\mathrm{H}(31 \mathrm{~A})$ & 0.9800 \\
\hline$C(3)-C(4)$ & $1.377(3)$ & $\mathrm{C}(31)-\mathrm{H}(31 \mathrm{~B})$ & 0.9800 \\
\hline $\mathrm{C}(3)-\mathrm{H}(3)$ & 0.9500 & $\mathrm{C}(31)-\mathrm{H}(31 \mathrm{C})$ & 0.9800 \\
\hline$C(4)-C(5)$ & $1.388(3)$ & $\mathrm{C}(32)-\mathrm{H}(32 \mathrm{~A})$ & 0.9800 \\
\hline $\mathrm{C}(4)-\mathrm{H}(4)$ & 0.9500 & $\mathrm{C}(32)-\mathrm{H}(32 \mathrm{~B})$ & 0.9800 \\
\hline$C(5)-C(6)$ & $1.382(3)$ & $\mathrm{C}(32)-\mathrm{H}(32 \mathrm{C})$ & 0.9800 \\
\hline $\mathrm{C}(5)-\mathrm{H}(5)$ & 0.9500 & $C(33)-C(36)$ & $1.516(3)$ \\
\hline $\mathrm{C}(6)-\mathrm{H}(6)$ & 0.9500 & $\mathrm{C}(33)-\mathrm{C}(35)$ & $1.523(3)$ \\
\hline $\mathrm{C}(7)-\mathrm{C}(12)$ & $1.398(3)$ & $\mathrm{C}(33)-\mathrm{C}(34)$ & $1.525(3)$ \\
\hline$C(7)-C(8)$ & $1.406(3)$ & $\mathrm{C}(34)-\mathrm{H}(34 \mathrm{~A})$ & 0.9800 \\
\hline $\mathrm{C}(8)-\mathrm{C}(9)$ & $1.393(3)$ & $\mathrm{C}(34)-\mathrm{H}(34 \mathrm{~B})$ & 0.9800 \\
\hline $\mathrm{C}(9)-\mathrm{C}(10)$ & $1.384(3)$ & $\mathrm{C}(34)-\mathrm{H}(34 \mathrm{C})$ & 0.9800 \\
\hline $\mathrm{C}(9)-\mathrm{H}(9)$ & 0.9500 & $\mathrm{C}(35)-\mathrm{H}(35 \mathrm{~A})$ & 0.9800 \\
\hline C(10)-C(11) & $1.386(3)$ & $\mathrm{C}(35)-\mathrm{H}(35 \mathrm{~B})$ & 0.9800 \\
\hline $\mathrm{C}(10)-\mathrm{H}(10)$ & 0.9500 & $\mathrm{C}(35)-\mathrm{H}(35 \mathrm{C})$ & 0.9800 \\
\hline $\mathrm{C}(11)-\mathrm{C}(12)$ & $1.375(3)$ & $\mathrm{C}(36)-\mathrm{H}(36 \mathrm{~A})$ & 0.9800 \\
\hline $\mathrm{C}(11)-\mathrm{H}(11)$ & 0.9500 & $\mathrm{C}(36)-\mathrm{H}(36 \mathrm{~B})$ & 0.9800 \\
\hline $\mathrm{C}(12)-\mathrm{H}(12)$ & 0.9500 & $\mathrm{C}(36)-\mathrm{H}(36 \mathrm{C})$ & 0.9800 \\
\hline C(13)-C(18) & $1.398(3)$ & $C(37)-C(39)$ & $1.517(3)$ \\
\hline C(13)-C(14) & $1.407(3)$ & C(37)-C(38) & $1.518(3)$ \\
\hline C(14)-C(15) & $1.390(3)$ & $\mathrm{C}(37)-\mathrm{C}(40)$ & $1.524(3)$ \\
\hline$C(15)-C(16)$ & $1.377(3)$ & $\mathrm{C}(38)-\mathrm{H}(38 \mathrm{~A})$ & 0.9800 \\
\hline $\mathrm{C}(15)-\mathrm{H}(15)$ & 0.9500 & $\mathrm{C}(38)-\mathrm{H}(38 \mathrm{~B})$ & 0.9800 \\
\hline C(16)-C(17) & $1.386(3)$ & $\mathrm{C}(38)-\mathrm{H}(38 \mathrm{C})$ & 0.9800 \\
\hline $\mathrm{C}(16)-\mathrm{H}(16)$ & 0.9500 & $\mathrm{C}(39)-\mathrm{H}(39 \mathrm{~A})$ & 0.9800 \\
\hline
\end{tabular}




\begin{tabular}{|c|c|c|c|}
\hline C(39)-H(39B) & 0.9800 & $\mathrm{C}(64)-\mathrm{F}(18)$ & $1.348(2)$ \\
\hline $\mathrm{C}(39)-\mathrm{H}(39 \mathrm{C})$ & 0.9800 & $C(65)-C(70)$ & $1.398(3)$ \\
\hline $\mathrm{C}(40)-\mathrm{H}(40 \mathrm{~A})$ & 0.9800 & $\mathrm{C}(65)-\mathrm{C}(66)$ & $1.406(3)$ \\
\hline $\mathrm{C}(40)-\mathrm{H}(40 \mathrm{~B})$ & 0.9800 & $\mathrm{C}(66)-\mathrm{C}(67)$ & $1.387(3)$ \\
\hline $\mathrm{C}(40)-\mathrm{H}(40 \mathrm{C})$ & 0.9800 & $\mathrm{C}(66)-\mathrm{H}(66)$ & 0.9500 \\
\hline $\mathrm{C}(41)-\mathrm{C}(42)$ & $1.398(3)$ & $\mathrm{C}(67)-\mathrm{C}(68)$ & $1.382(3)$ \\
\hline $\mathrm{C}(41)-\mathrm{C}(46)$ & $1.408(3)$ & $\mathrm{C}(67)-\mathrm{C}(71)$ & $1.500(3)$ \\
\hline$C(42)-C(43)$ & $1.385(3)$ & $\mathrm{C}(68)-\mathrm{C}(69)$ & $1.388(3)$ \\
\hline $\mathrm{C}(42)-\mathrm{H}(42)$ & 0.9500 & $\mathrm{C}(68)-\mathrm{H}(68)$ & 0.9500 \\
\hline C(43)-C(44) & $1.390(3)$ & $\mathrm{C}(69)-\mathrm{C}(70)$ & $1.393(3)$ \\
\hline $\mathrm{C}(43)-\mathrm{C}(47)$ & $1.492(3)$ & $\mathrm{C}(69)-\mathrm{C}(72)$ & $1.489(3)$ \\
\hline$C(44)-C(45)$ & $1.385(3)$ & $\mathrm{C}(70)-\mathrm{H}(70)$ & 0.9500 \\
\hline $\mathrm{C}(44)-\mathrm{H}(44)$ & 0.9500 & $\mathrm{C}(71)-\mathrm{F}(21)$ & $1.325(3)$ \\
\hline $\mathrm{C}(45)-\mathrm{C}(46)$ & $1.387(3)$ & $\mathrm{C}(71)-\mathrm{F}(19)$ & $1.326(3)$ \\
\hline $\mathrm{C}(45)-\mathrm{C}(48)$ & $1.498(3)$ & $\mathrm{C}(71)-\mathrm{F}(20)$ & $1.327(3)$ \\
\hline $\mathrm{C}(46)-\mathrm{H}(46)$ & 0.9500 & $\mathrm{C}(72)-\mathrm{F}(23)$ & $1.334(3)$ \\
\hline$C(47)-F(1)$ & $1.329(2)$ & $\mathrm{C}(72)-\mathrm{F}(24)$ & $1.342(3)$ \\
\hline$C(47)-F(2)$ & $1.337(2)$ & $C(72)-F(22)$ & $1.344(2)$ \\
\hline$C(47)-F(3)$ & $1.346(3)$ & $C(73)-C(75)$ & $1.716(13)$ \\
\hline$C(48)-F(6 A)$ & $1.16(2)$ & $\mathrm{C}(73)-\mathrm{H}(73 \mathrm{~A})$ & 0.9900 \\
\hline $\mathrm{C}(48)-\mathrm{F}(4 \mathrm{~A})$ & $1.244(17)$ & $\mathrm{C}(73)-\mathrm{H}(73 \mathrm{~B})$ & 0.9900 \\
\hline $\mathrm{C}(48)-\mathrm{F}(5 \mathrm{~A})$ & $1.293(19)$ & $\mathrm{C}(74)-\mathrm{C}(75)$ & $1.237(8)$ \\
\hline$C(48)-F(6)$ & $1.298(3)$ & $\mathrm{C}(74)-\mathrm{H}(74 \mathrm{~A})$ & 0.9900 \\
\hline$C(48)-F(5)$ & $1.320(3)$ & $\mathrm{C}(74)-\mathrm{H}(74 \mathrm{~B})$ & 0.9900 \\
\hline$C(48)-F(4)$ & $1.333(3)$ & $\mathrm{C}(74)-\mathrm{H}(75 \mathrm{E})$ & 1.4650 \\
\hline $\mathrm{C}(49)-\mathrm{C}(50)$ & $1.399(3)$ & $\mathrm{C}(74)-\mathrm{H}(75 \mathrm{~F})$ & 1.4689 \\
\hline $\mathrm{C}(49)-\mathrm{C}(54)$ & $1.401(3)$ & $\mathrm{C}(75)-\mathrm{H}(75 \mathrm{~A})$ & 1.0045 \\
\hline$C(50)-C(51)$ & 1.391(3) & $\mathrm{C}(75)-\mathrm{H}(75 \mathrm{~B})$ & 0.9844 \\
\hline $\mathrm{C}(50)-\mathrm{H}(50)$ & 0.9500 & $\mathrm{C}(75)-\mathrm{H}(75 \mathrm{C})$ & 0.9865 \\
\hline $\mathrm{C}(51)-\mathrm{C}(52)$ & $1.377(3)$ & $\mathrm{C}(75)-\mathrm{H}(75 \mathrm{D})$ & 1.0401 \\
\hline$C(51)-C(55)$ & $1.494(3)$ & $\mathrm{C}(75)-\mathrm{H}(75 \mathrm{E})$ & 0.9524 \\
\hline$C(52)-C(53)$ & $1.381(3)$ & $\mathrm{C}(75)-\mathrm{H}(75 \mathrm{~F})$ & 0.9543 \\
\hline $\mathrm{C}(52)-\mathrm{H}(52)$ & 0.9500 & $\mathrm{~N}(1)-\mathrm{Cu}(1)-\mathrm{N}(2)$ & $105.53(6)$ \\
\hline $\mathrm{C}(53)-\mathrm{C}(54)$ & $1.390(3)$ & $\mathrm{N}(1)-\mathrm{Cu}(1)-\mathrm{S}(2)$ & $87.76(5)$ \\
\hline C(53)-C(56) & $1.494(3)$ & $\mathrm{N}(2)-\mathrm{Cu}(1)-\mathrm{S}(2)$ & $108.41(5)$ \\
\hline $\mathrm{C}(54)-\mathrm{H}(54)$ & 0.9500 & $\mathrm{~N}(1)-\mathrm{Cu}(1)-\mathrm{S}(3)$ & $112.57(5)$ \\
\hline $\mathrm{C}(55)-\mathrm{F}(9)$ & $1.318(3)$ & $\mathrm{N}(2)-\mathrm{Cu}(1)-\mathrm{S}(3)$ & $87.25(5)$ \\
\hline $\mathrm{C}(55)-\mathrm{F}(8)$ & $1.318(3)$ & $\mathrm{S}(2)-\mathrm{Cu}(1)-\mathrm{S}(3)$ & $150.50(2)$ \\
\hline$C(55)-F(7)$ & $1.356(3)$ & $\mathrm{N}(2)-\mathrm{Cu}(2)-\mathrm{N}(1)$ & 108.09(6) \\
\hline $\mathrm{C}(56)-\mathrm{F}(11)$ & $1.328(3)$ & $\mathrm{N}(2)-\mathrm{Cu}(2)-\mathrm{S}(4)$ & $88.22(5)$ \\
\hline $\mathrm{C}(56)-\mathrm{F}(12)$ & $1.332(3)$ & $\mathrm{N}(1)-\mathrm{Cu}(2)-\mathrm{S}(4)$ & $108.04(5)$ \\
\hline$C(56)-F(10)$ & $1.337(3)$ & $\mathrm{N}(2)-\mathrm{Cu}(2)-\mathrm{S}(1)$ & $110.73(5)$ \\
\hline C(57)-C(58) & $1.391(3)$ & $\mathrm{N}(1)-\mathrm{Cu}(2)-\mathrm{S}(1)$ & $87.69(5)$ \\
\hline $\mathrm{C}(57)-\mathrm{C}(62)$ & $1.405(3)$ & $\mathrm{S}(4)-\mathrm{Cu}(2)-\mathrm{S}(1)$ & $150.71(2)$ \\
\hline $\mathrm{C}(58)-\mathrm{C}(59)$ & $1.395(3)$ & $\mathrm{C}(1)-\mathrm{N}(1)-\mathrm{C}(7)$ & $118.48(16)$ \\
\hline $\mathrm{C}(58)-\mathrm{H}(58)$ & 0.9500 & $\mathrm{C}(1)-\mathrm{N}(1)-\mathrm{Cu}(2)$ & $115.36(13)$ \\
\hline $\mathrm{C}(59)-\mathrm{C}(60)$ & $1.384(3)$ & $\mathrm{C}(7)-\mathrm{N}(1)-\mathrm{Cu}(2)$ & $113.35(12)$ \\
\hline $\mathrm{C}(59)-\mathrm{C}(63)$ & $1.494(3)$ & $\mathrm{C}(1)-\mathrm{N}(1)-\mathrm{Cu}(1)$ & $113.92(12)$ \\
\hline $\mathrm{C}(60)-\mathrm{C}(61)$ & $1.383(3)$ & $\mathrm{C}(7)-\mathrm{N}(1)-\mathrm{Cu}(1)$ & $114.44(12)$ \\
\hline $\mathrm{C}(60)-\mathrm{H}(60)$ & 0.9500 & $\mathrm{Cu}(2)-\mathrm{N}(1)-\mathrm{Cu}(1)$ & $73.07(5)$ \\
\hline$C(61)-C(62)$ & $1.390(3)$ & $\mathrm{C}(19)-\mathrm{N}(2)-\mathrm{C}(13)$ & $119.09(16)$ \\
\hline $\mathrm{C}(61)-\mathrm{C}(64)$ & $1.494(3)$ & $\mathrm{C}(19)-\mathrm{N}(2)-\mathrm{Cu}(2)$ & $114.69(12)$ \\
\hline $\mathrm{C}(62)-\mathrm{H}(62)$ & 0.9500 & $\mathrm{C}(13)-\mathrm{N}(2)-\mathrm{Cu}(2)$ & $114.79(12)$ \\
\hline $\mathrm{C}(63)-\mathrm{F}(13)$ & $1.333(2)$ & $\mathrm{C}(19)-\mathrm{N}(2)-\mathrm{Cu}(1)$ & $111.81(12)$ \\
\hline$C(63)-F(14)$ & $1.334(2)$ & $\mathrm{C}(13)-\mathrm{N}(2)-\mathrm{Cu}(1)$ & $114.85(13)$ \\
\hline $\mathrm{C}(63)-\mathrm{F}(15)$ & $1.336(3)$ & $\mathrm{Cu}(2)-\mathrm{N}(2)-\mathrm{Cu}(1)$ & $72.96(5)$ \\
\hline $\mathrm{C}(64)-\mathrm{F}(16)$ & $1.335(2)$ & $\mathrm{C}(2)-\mathrm{S}(1)-\mathrm{C}(25)$ & $103.85(10)$ \\
\hline$C(64)-F(17)$ & $1.341(2)$ & $\mathrm{C}(2)-\mathrm{S}(1)-\mathrm{Cu}(2)$ & $96.78(7)$ \\
\hline
\end{tabular}




\begin{tabular}{|c|c|c|c|}
\hline $\mathrm{C}(25)-\mathrm{S}(1)-\mathrm{Cu}(2)$ & $108.99(8)$ & $\mathrm{C}(18)-\mathrm{C}(13)-\mathrm{C}(14)$ & $118.45(19)$ \\
\hline $\mathrm{C}(8)-\mathrm{S}(2)-\mathrm{C}(29)$ & $104.32(10)$ & $\mathrm{C}(18)-\mathrm{C}(13)-\mathrm{N}(2)$ & $122.09(19)$ \\
\hline $\mathrm{C}(8)-\mathrm{S}(2)-\mathrm{Cu}(1)$ & $96.64(7)$ & $\mathrm{C}(14)-\mathrm{C}(13)-\mathrm{N}(2)$ & $119.00(18)$ \\
\hline $\mathrm{C}(29)-\mathrm{S}(2)-\mathrm{Cu}(1)$ & $111.48(7)$ & $\mathrm{C}(15)-\mathrm{C}(14)-\mathrm{C}(13)$ & $119.8(2)$ \\
\hline $\mathrm{C}(14)-\mathrm{S}(3)-\mathrm{C}(33)$ & $102.30(10)$ & $C(15)-C(14)-S(3)$ & $120.05(17)$ \\
\hline $\mathrm{C}(14)-\mathrm{S}(3)-\mathrm{Cu}(1)$ & $97.25(7)$ & $\mathrm{C}(13)-\mathrm{C}(14)-\mathrm{S}(3)$ & $120.15(16)$ \\
\hline $\mathrm{C}(33)-\mathrm{S}(3)-\mathrm{Cu}(1)$ & $109.99(7)$ & $\mathrm{C}(16)-\mathrm{C}(15)-\mathrm{C}(14)$ & $120.8(2)$ \\
\hline$C(20)-S(4)-C(37)$ & $102.34(10)$ & $\mathrm{C}(16)-\mathrm{C}(15)-\mathrm{H}(15)$ & 119.6 \\
\hline $\mathrm{C}(20)-\mathrm{S}(4)-\mathrm{Cu}(2)$ & $95.40(7)$ & $\mathrm{C}(14)-\mathrm{C}(15)-\mathrm{H}(15)$ & 119.6 \\
\hline $\mathrm{C}(37)-\mathrm{S}(4)-\mathrm{Cu}(2)$ & $112.84(8)$ & $C(15)-C(16)-C(17)$ & $119.5(2)$ \\
\hline $\mathrm{C}(73)-\mathrm{O}-\mathrm{C}(73) \# 1$ & $180.0(12)$ & $\mathrm{C}(15)-\mathrm{C}(16)-\mathrm{H}(16)$ & 120.2 \\
\hline $\mathrm{C}(73)-\mathrm{O}-\mathrm{C}(74)$ & $58.2(8)$ & $\mathrm{C}(17)-\mathrm{C}(16)-\mathrm{H}(16)$ & 120.2 \\
\hline C(73)\#1-O-C(74) & $121.8(8)$ & $\mathrm{C}(18)-\mathrm{C}(17)-\mathrm{C}(16)$ & $120.5(2)$ \\
\hline $\mathrm{C}(73)-\mathrm{O}-\mathrm{C}(74) \# 1$ & $121.8(8)$ & $\mathrm{C}(18)-\mathrm{C}(17)-\mathrm{H}(17)$ & 119.7 \\
\hline $\mathrm{C}(73) \# 1-\mathrm{O}-\mathrm{C}(74) \# 1$ & $58.2(8)$ & $\mathrm{C}(16)-\mathrm{C}(17)-\mathrm{H}(17)$ & 119.7 \\
\hline C(74)-O-C(74)\#1 & $180.0(4)$ & $\mathrm{C}(17)-\mathrm{C}(18)-\mathrm{C}(13)$ & $120.5(2)$ \\
\hline $\mathrm{C}(65)-\mathrm{B}(1)-\mathrm{C}(49)$ & $110.28(16)$ & $\mathrm{C}(17)-\mathrm{C}(18)-\mathrm{H}(18)$ & 119.8 \\
\hline $\mathrm{C}(65)-\mathrm{B}(1)-\mathrm{C}(41)$ & $108.35(16)$ & $\mathrm{C}(13)-\mathrm{C}(18)-\mathrm{H}(18)$ & 119.8 \\
\hline $\mathrm{C}(49)-\mathrm{B}(1)-\mathrm{C}(41)$ & $109.65(16)$ & $\mathrm{C}(24)-\mathrm{C}(19)-\mathrm{N}(2)$ & $122.43(18)$ \\
\hline $\mathrm{C}(65)-\mathrm{B}(1)-\mathrm{C}(57)$ & $110.87(16)$ & $C(24)-C(19)-C(20)$ & $117.88(19)$ \\
\hline $\mathrm{C}(49)-\mathrm{B}(1)-\mathrm{C}(57)$ & 109.43(16) & $\mathrm{N}(2)-\mathrm{C}(19)-\mathrm{C}(20)$ & $119.17(18)$ \\
\hline $\mathrm{C}(41)-\mathrm{B}(1)-\mathrm{C}(57)$ & $108.23(16)$ & $\mathrm{C}(21)-\mathrm{C}(20)-\mathrm{C}(19)$ & $120.03(19)$ \\
\hline$C(6)-C(1)-C(2)$ & $118.20(19)$ & $C(21)-C(20)-S(4)$ & $119.93(16)$ \\
\hline $\mathrm{C}(6)-\mathrm{C}(1)-\mathrm{N}(1)$ & $121.95(18)$ & $C(19)-C(20)-S(4)$ & $120.04(16)$ \\
\hline $\mathrm{C}(2)-\mathrm{C}(1)-\mathrm{N}(1)$ & $119.37(18)$ & $\mathrm{C}(22)-\mathrm{C}(21)-\mathrm{C}(20)$ & $120.6(2)$ \\
\hline $\mathrm{C}(3)-\mathrm{C}(2)-\mathrm{C}(1)$ & $120.0(2)$ & $\mathrm{C}(22)-\mathrm{C}(21)-\mathrm{H}(21)$ & 119.7 \\
\hline $\mathrm{C}(3)-\mathrm{C}(2)-\mathrm{S}(1)$ & $120.32(17)$ & $\mathrm{C}(20)-\mathrm{C}(21)-\mathrm{H}(21)$ & 119.7 \\
\hline$C(1)-C(2)-S(1)$ & $119.71(16)$ & $\mathrm{C}(21)-\mathrm{C}(22)-\mathrm{C}(23)$ & $119.7(2)$ \\
\hline$C(4)-C(3)-C(2)$ & $121.0(2)$ & $\mathrm{C}(21)-\mathrm{C}(22)-\mathrm{H}(22)$ & 120.1 \\
\hline $\mathrm{C}(4)-\mathrm{C}(3)-\mathrm{H}(3)$ & 119.5 & $\mathrm{C}(23)-\mathrm{C}(22)-\mathrm{H}(22)$ & 120.1 \\
\hline $\mathrm{C}(2)-\mathrm{C}(3)-\mathrm{H}(3)$ & 119.5 & $\mathrm{C}(24)-\mathrm{C}(23)-\mathrm{C}(22)$ & $120.5(2)$ \\
\hline$C(3)-C(4)-C(5)$ & $119.4(2)$ & $\mathrm{C}(24)-\mathrm{C}(23)-\mathrm{H}(23)$ & 119.8 \\
\hline $\mathrm{C}(3)-\mathrm{C}(4)-\mathrm{H}(4)$ & 120.3 & $\mathrm{C}(22)-\mathrm{C}(23)-\mathrm{H}(23)$ & 119.8 \\
\hline $\mathrm{C}(5)-\mathrm{C}(4)-\mathrm{H}(4)$ & 120.3 & $\mathrm{C}(23)-\mathrm{C}(24)-\mathrm{C}(19)$ & $120.9(2)$ \\
\hline$C(6)-C(5)-C(4)$ & $120.4(2)$ & $\mathrm{C}(23)-\mathrm{C}(24)-\mathrm{H}(24)$ & 119.5 \\
\hline $\mathrm{C}(6)-\mathrm{C}(5)-\mathrm{H}(5)$ & 119.8 & $\mathrm{C}(19)-\mathrm{C}(24)-\mathrm{H}(24)$ & 119.5 \\
\hline $\mathrm{C}(4)-\mathrm{C}(5)-\mathrm{H}(5)$ & 119.8 & $\mathrm{C}(28)-\mathrm{C}(25)-\mathrm{C}(26)$ & $111.6(2)$ \\
\hline$C(5)-C(6)-C(1)$ & $120.7(2)$ & $\mathrm{C}(28)-\mathrm{C}(25)-\mathrm{C}(27)$ & $110.88(19)$ \\
\hline $\mathrm{C}(5)-\mathrm{C}(6)-\mathrm{H}(6)$ & 119.6 & $\mathrm{C}(26)-\mathrm{C}(25)-\mathrm{C}(27)$ & $110.7(2)$ \\
\hline $\mathrm{C}(1)-\mathrm{C}(6)-\mathrm{H}(6)$ & 119.6 & $\mathrm{C}(28)-\mathrm{C}(25)-\mathrm{S}(1)$ & $110.40(15)$ \\
\hline $\mathrm{C}(12)-\mathrm{C}(7)-\mathrm{C}(8)$ & $118.23(18)$ & $\mathrm{C}(26)-\mathrm{C}(25)-\mathrm{S}(1)$ & $103.49(15)$ \\
\hline $\mathrm{C}(12)-\mathrm{C}(7)-\mathrm{N}(1)$ & $121.59(18)$ & $C(27)-C(25)-S(1)$ & $109.56(17)$ \\
\hline $\mathrm{C}(8)-\mathrm{C}(7)-\mathrm{N}(1)$ & 119.71(17) & $\mathrm{C}(25)-\mathrm{C}(26)-\mathrm{H}(26 \mathrm{~A})$ & 109.5 \\
\hline $\mathrm{C}(9)-\mathrm{C}(8)-\mathrm{C}(7)$ & $119.88(19)$ & $\mathrm{C}(25)-\mathrm{C}(26)-\mathrm{H}(26 \mathrm{~B})$ & 109.5 \\
\hline $\mathrm{C}(9)-\mathrm{C}(8)-\mathrm{S}(2)$ & $120.00(16)$ & $\mathrm{H}(26 \mathrm{~A})-\mathrm{C}(26)-\mathrm{H}(26 \mathrm{~B})$ & 109.5 \\
\hline $\mathrm{C}(7)-\mathrm{C}(8)-\mathrm{S}(2)$ & $120.09(15)$ & $\mathrm{C}(25)-\mathrm{C}(26)-\mathrm{H}(26 \mathrm{C})$ & 109.5 \\
\hline $\mathrm{C}(10)-\mathrm{C}(9)-\mathrm{C}(8)$ & $120.8(2)$ & $\mathrm{H}(26 \mathrm{~A})-\mathrm{C}(26)-\mathrm{H}(26 \mathrm{C})$ & 109.5 \\
\hline $\mathrm{C}(10)-\mathrm{C}(9)-\mathrm{H}(9)$ & 119.6 & $\mathrm{H}(26 \mathrm{~B})-\mathrm{C}(26)-\mathrm{H}(26 \mathrm{C})$ & 109.5 \\
\hline $\mathrm{C}(8)-\mathrm{C}(9)-\mathrm{H}(9)$ & 119.6 & $\mathrm{C}(25)-\mathrm{C}(27)-\mathrm{H}(27 \mathrm{~A})$ & 109.5 \\
\hline$C(9)-C(10)-C(11)$ & $119.2(2)$ & $\mathrm{C}(25)-\mathrm{C}(27)-\mathrm{H}(27 \mathrm{~B})$ & 109.5 \\
\hline $\mathrm{C}(9)-\mathrm{C}(10)-\mathrm{H}(10)$ & 120.4 & $\mathrm{H}(27 \mathrm{~A})-\mathrm{C}(27)-\mathrm{H}(27 \mathrm{~B})$ & 109.5 \\
\hline $\mathrm{C}(11)-\mathrm{C}(10)-\mathrm{H}(10)$ & 120.4 & $\mathrm{C}(25)-\mathrm{C}(27)-\mathrm{H}(27 \mathrm{C})$ & 109.5 \\
\hline $\mathrm{C}(12)-\mathrm{C}(11)-\mathrm{C}(10)$ & $120.7(2)$ & $\mathrm{H}(27 \mathrm{~A})-\mathrm{C}(27)-\mathrm{H}(27 \mathrm{C})$ & 109.5 \\
\hline $\mathrm{C}(12)-\mathrm{C}(11)-\mathrm{H}(11)$ & 119.6 & H(27B)-C(27)-H(27C) & 109.5 \\
\hline $\mathrm{C}(10)-\mathrm{C}(11)-\mathrm{H}(11)$ & 119.6 & $\mathrm{C}(25)-\mathrm{C}(28)-\mathrm{H}(28 \mathrm{~A})$ & 109.5 \\
\hline $\mathrm{C}(11)-\mathrm{C}(12)-\mathrm{C}(7)$ & $120.9(2)$ & $\mathrm{C}(25)-\mathrm{C}(28)-\mathrm{H}(28 \mathrm{~B})$ & 109.5 \\
\hline $\mathrm{C}(11)-\mathrm{C}(12)-\mathrm{H}(12)$ & 119.5 & $\mathrm{H}(28 \mathrm{~A})-\mathrm{C}(28)-\mathrm{H}(28 \mathrm{~B})$ & 109.5 \\
\hline $\mathrm{C}(7)-\mathrm{C}(12)-\mathrm{H}(12)$ & 119.5 & $\mathrm{C}(25)-\mathrm{C}(28)-\mathrm{H}(28 \mathrm{C})$ & 109.5 \\
\hline
\end{tabular}




\begin{tabular}{|c|c|c|c|}
\hline $\mathrm{H}(28 \mathrm{~A})-\mathrm{C}(28)-\mathrm{H}(28 \mathrm{C})$ & 109.5 & $\mathrm{H}(38 \mathrm{~A})-\mathrm{C}(38)-\mathrm{H}(38 \mathrm{~B})$ & 109.5 \\
\hline $\mathrm{H}(28 \mathrm{~B})-\mathrm{C}(28)-\mathrm{H}(28 \mathrm{C})$ & 109.5 & $\mathrm{C}(37)-\mathrm{C}(38)-\mathrm{H}(38 \mathrm{C})$ & 109.5 \\
\hline$C(31)-C(29)-C(30)$ & $112.78(19)$ & $\mathrm{H}(38 \mathrm{~A})-\mathrm{C}(38)-\mathrm{H}(38 \mathrm{C})$ & 109.5 \\
\hline $\mathrm{C}(31)-\mathrm{C}(29)-\mathrm{C}(32)$ & $110.45(19)$ & H(38B)-C(38)-H(38C) & 109.5 \\
\hline $\mathrm{C}(30)-\mathrm{C}(29)-\mathrm{C}(32)$ & 109.98(19) & $\mathrm{C}(37)-\mathrm{C}(39)-\mathrm{H}(39 \mathrm{~A})$ & 109.5 \\
\hline $\mathrm{C}(31)-\mathrm{C}(29)-\mathrm{S}(2)$ & $110.45(15)$ & $\mathrm{C}(37)-\mathrm{C}(39)-\mathrm{H}(39 \mathrm{~B})$ & 109.5 \\
\hline $\mathrm{C}(30)-\mathrm{C}(29)-\mathrm{S}(2)$ & $103.21(15)$ & $\mathrm{H}(39 \mathrm{~A})-\mathrm{C}(39)-\mathrm{H}(39 \mathrm{~B})$ & 109.5 \\
\hline$C(32)-C(29)-S(2)$ & $109.74(15)$ & $\mathrm{C}(37)-\mathrm{C}(39)-\mathrm{H}(39 \mathrm{C})$ & 109.5 \\
\hline $\mathrm{C}(29)-\mathrm{C}(30)-\mathrm{H}(30 \mathrm{~A})$ & 109.5 & $\mathrm{H}(39 \mathrm{~A})-\mathrm{C}(39)-\mathrm{H}(39 \mathrm{C})$ & 109.5 \\
\hline $\mathrm{C}(29)-\mathrm{C}(30)-\mathrm{H}(30 \mathrm{~B})$ & 109.5 & H(39B)-C(39)-H(39C) & 109.5 \\
\hline $\mathrm{H}(30 \mathrm{~A})-\mathrm{C}(30)-\mathrm{H}(30 \mathrm{~B})$ & 109.5 & $\mathrm{C}(37)-\mathrm{C}(40)-\mathrm{H}(40 \mathrm{~A})$ & 109.5 \\
\hline $\mathrm{C}(29)-\mathrm{C}(30)-\mathrm{H}(30 \mathrm{C})$ & 109.5 & $\mathrm{C}(37)-\mathrm{C}(40)-\mathrm{H}(40 \mathrm{~B})$ & 109.5 \\
\hline $\mathrm{H}(30 \mathrm{~A})-\mathrm{C}(30)-\mathrm{H}(30 \mathrm{C})$ & 109.5 & $\mathrm{H}(40 \mathrm{~A})-\mathrm{C}(40)-\mathrm{H}(40 \mathrm{~B})$ & 109.5 \\
\hline $\mathrm{H}(30 \mathrm{~B})-\mathrm{C}(30)-\mathrm{H}(30 \mathrm{C})$ & 109.5 & $\mathrm{C}(37)-\mathrm{C}(40)-\mathrm{H}(40 \mathrm{C})$ & 109.5 \\
\hline $\mathrm{C}(29)-\mathrm{C}(31)-\mathrm{H}(31 \mathrm{~A})$ & 109.5 & $\mathrm{H}(40 \mathrm{~A})-\mathrm{C}(40)-\mathrm{H}(40 \mathrm{C})$ & 109.5 \\
\hline $\mathrm{C}(29)-\mathrm{C}(31)-\mathrm{H}(31 \mathrm{~B})$ & 109.5 & $\mathrm{H}(40 \mathrm{~B})-\mathrm{C}(40)-\mathrm{H}(40 \mathrm{C})$ & 109.5 \\
\hline $\mathrm{H}(31 \mathrm{~A})-\mathrm{C}(31)-\mathrm{H}(31 \mathrm{~B})$ & 109.5 & $\mathrm{C}(42)-\mathrm{C}(41)-\mathrm{C}(46)$ & $115.17(18)$ \\
\hline $\mathrm{C}(29)-\mathrm{C}(31)-\mathrm{H}(31 \mathrm{C})$ & 109.5 & $\mathrm{C}(42)-\mathrm{C}(41)-\mathrm{B}(1)$ & $123.79(17)$ \\
\hline $\mathrm{H}(31 \mathrm{~A})-\mathrm{C}(31)-\mathrm{H}(31 \mathrm{C})$ & 109.5 & $\mathrm{C}(46)-\mathrm{C}(41)-\mathrm{B}(1)$ & $121.04(18)$ \\
\hline $\mathrm{H}(31 \mathrm{~B})-\mathrm{C}(31)-\mathrm{H}(31 \mathrm{C})$ & 109.5 & $\mathrm{C}(43)-\mathrm{C}(42)-\mathrm{C}(41)$ & $122.75(19)$ \\
\hline $\mathrm{C}(29)-\mathrm{C}(32)-\mathrm{H}(32 \mathrm{~A})$ & 109.5 & $\mathrm{C}(43)-\mathrm{C}(42)-\mathrm{H}(42)$ & 118.6 \\
\hline $\mathrm{C}(29)-\mathrm{C}(32)-\mathrm{H}(32 \mathrm{~B})$ & 109.5 & $\mathrm{C}(41)-\mathrm{C}(42)-\mathrm{H}(42)$ & 118.6 \\
\hline $\mathrm{H}(32 \mathrm{~A})-\mathrm{C}(32)-\mathrm{H}(32 \mathrm{~B})$ & 109.5 & $C(42)-C(43)-C(44)$ & $120.67(19)$ \\
\hline $\mathrm{C}(29)-\mathrm{C}(32)-\mathrm{H}(32 \mathrm{C})$ & 109.5 & $C(42)-C(43)-C(47)$ & $121.27(18)$ \\
\hline $\mathrm{H}(32 \mathrm{~A})-\mathrm{C}(32)-\mathrm{H}(32 \mathrm{C})$ & 109.5 & $\mathrm{C}(44)-\mathrm{C}(43)-\mathrm{C}(47)$ & $118.03(18)$ \\
\hline $\mathrm{H}(32 \mathrm{~B})-\mathrm{C}(32)-\mathrm{H}(32 \mathrm{C})$ & 109.5 & $\mathrm{C}(45)-\mathrm{C}(44)-\mathrm{C}(43)$ & $118.20(19)$ \\
\hline $\mathrm{C}(36)-\mathrm{C}(33)-\mathrm{C}(35)$ & $110.35(19)$ & $\mathrm{C}(45)-\mathrm{C}(44)-\mathrm{H}(44)$ & 120.9 \\
\hline $\mathrm{C}(36)-\mathrm{C}(33)-\mathrm{C}(34)$ & $112.2(2)$ & $\mathrm{C}(43)-\mathrm{C}(44)-\mathrm{H}(44)$ & 120.9 \\
\hline $\mathrm{C}(35)-\mathrm{C}(33)-\mathrm{C}(34)$ & $110.66(18)$ & $\mathrm{C}(44)-\mathrm{C}(45)-\mathrm{C}(46)$ & $120.61(19)$ \\
\hline $\mathrm{C}(36)-\mathrm{C}(33)-\mathrm{S}(3)$ & $103.85(14)$ & $\mathrm{C}(44)-\mathrm{C}(45)-\mathrm{C}(48)$ & $119.16(19)$ \\
\hline $\mathrm{C}(35)-\mathrm{C}(33)-\mathrm{S}(3)$ & 109.71(16) & $\mathrm{C}(46)-\mathrm{C}(45)-\mathrm{C}(48)$ & $120.23(19)$ \\
\hline $\mathrm{C}(34)-\mathrm{C}(33)-\mathrm{S}(3)$ & $109.80(14)$ & $\mathrm{C}(45)-\mathrm{C}(46)-\mathrm{C}(41)$ & $122.60(19)$ \\
\hline $\mathrm{C}(33)-\mathrm{C}(34)-\mathrm{H}(34 \mathrm{~A})$ & 109.5 & $\mathrm{C}(45)-\mathrm{C}(46)-\mathrm{H}(46)$ & 118.7 \\
\hline $\mathrm{C}(33)-\mathrm{C}(34)-\mathrm{H}(34 \mathrm{~B})$ & 109.5 & $\mathrm{C}(41)-\mathrm{C}(46)-\mathrm{H}(46)$ & 118.7 \\
\hline $\mathrm{H}(34 \mathrm{~A})-\mathrm{C}(34)-\mathrm{H}(34 \mathrm{~B})$ & 109.5 & $\mathrm{~F}(1)-\mathrm{C}(47)-\mathrm{F}(2)$ & $106.98(18)$ \\
\hline $\mathrm{C}(33)-\mathrm{C}(34)-\mathrm{H}(34 \mathrm{C})$ & 109.5 & $\mathrm{~F}(1)-\mathrm{C}(47)-\mathrm{F}(3)$ & $105.92(18)$ \\
\hline $\mathrm{H}(34 \mathrm{~A})-\mathrm{C}(34)-\mathrm{H}(34 \mathrm{C})$ & 109.5 & $\mathrm{~F}(2)-\mathrm{C}(47)-\mathrm{F}(3)$ & $104.86(17)$ \\
\hline $\mathrm{H}(34 \mathrm{~B})-\mathrm{C}(34)-\mathrm{H}(34 \mathrm{C})$ & 109.5 & $\mathrm{~F}(1)-\mathrm{C}(47)-\mathrm{C}(43)$ & $113.92(18)$ \\
\hline $\mathrm{C}(33)-\mathrm{C}(35)-\mathrm{H}(35 \mathrm{~A})$ & 109.5 & $\mathrm{~F}(2)-\mathrm{C}(47)-\mathrm{C}(43)$ & $112.62(18)$ \\
\hline $\mathrm{C}(33)-\mathrm{C}(35)-\mathrm{H}(35 \mathrm{~B})$ & 109.5 & $\mathrm{~F}(3)-\mathrm{C}(47)-\mathrm{C}(43)$ & $111.89(18)$ \\
\hline $\mathrm{H}(35 \mathrm{~A})-\mathrm{C}(35)-\mathrm{H}(35 \mathrm{~B})$ & 109.5 & $\mathrm{~F}(6 \mathrm{~A})-\mathrm{C}(48)-\mathrm{F}(4 \mathrm{~A})$ & $102.3(14)$ \\
\hline $\mathrm{C}(33)-\mathrm{C}(35)-\mathrm{H}(35 \mathrm{C})$ & 109.5 & $\mathrm{~F}(6 \mathrm{~A})-\mathrm{C}(48)-\mathrm{F}(5 \mathrm{~A})$ & 103.0(14) \\
\hline $\mathrm{H}(35 \mathrm{~A})-\mathrm{C}(35)-\mathrm{H}(35 \mathrm{C})$ & 109.5 & $\mathrm{~F}(4 \mathrm{~A})-\mathrm{C}(48)-\mathrm{F}(5 \mathrm{~A})$ & $107.8(13)$ \\
\hline $\mathrm{H}(35 \mathrm{~B})-\mathrm{C}(35)-\mathrm{H}(35 \mathrm{C})$ & 109.5 & $\mathrm{~F}(6 \mathrm{~A})-\mathrm{C}(48)-\mathrm{F}(6)$ & $125.1(10)$ \\
\hline $\mathrm{C}(33)-\mathrm{C}(36)-\mathrm{H}(36 \mathrm{~A})$ & 109.5 & $\mathrm{~F}(4 \mathrm{~A})-\mathrm{C}(48)-\mathrm{F}(6)$ & $50.5(9)$ \\
\hline $\mathrm{C}(33)-\mathrm{C}(36)-\mathrm{H}(36 \mathrm{~B})$ & 109.5 & $\mathrm{~F}(5 \mathrm{~A})-\mathrm{C}(48)-\mathrm{F}(6)$ & $59.6(10)$ \\
\hline $\mathrm{H}(36 \mathrm{~A})-\mathrm{C}(36)-\mathrm{H}(36 \mathrm{~B})$ & 109.5 & $F(6 A)-C(48)-F(5)$ & $55.6(12)$ \\
\hline $\mathrm{C}(33)-\mathrm{C}(36)-\mathrm{H}(36 \mathrm{C})$ & 109.5 & $\mathrm{~F}(4 \mathrm{~A})-\mathrm{C}(48)-\mathrm{F}(5)$ & $135.1(8)$ \\
\hline $\mathrm{H}(36 \mathrm{~A})-\mathrm{C}(36)-\mathrm{H}(36 \mathrm{C})$ & 109.5 & $\mathrm{~F}(5 \mathrm{~A})-\mathrm{C}(48)-\mathrm{F}(5)$ & $53.1(10)$ \\
\hline $\mathrm{H}(36 \mathrm{~B})-\mathrm{C}(36)-\mathrm{H}(36 \mathrm{C})$ & 109.5 & $\mathrm{~F}(6)-\mathrm{C}(48)-\mathrm{F}(5)$ & $106.8(2)$ \\
\hline$C(39)-C(37)-C(38)$ & $111.3(2)$ & $\mathrm{F}(6 \mathrm{~A})-\mathrm{C}(48)-\mathrm{F}(4)$ & $47.7(12)$ \\
\hline $\mathrm{C}(39)-\mathrm{C}(37)-\mathrm{C}(40)$ & $111.9(2)$ & $\mathrm{F}(4 \mathrm{~A})-\mathrm{C}(48)-\mathrm{F}(4)$ & $61.9(9)$ \\
\hline $\mathrm{C}(38)-\mathrm{C}(37)-\mathrm{C}(40)$ & $110.5(2)$ & $\mathrm{F}(5 \mathrm{~A})-\mathrm{C}(48)-\mathrm{F}(4)$ & 137.1(9) \\
\hline C(39)-C(37)-S(4) & $110.84(15)$ & $\mathrm{F}(6)-\mathrm{C}(48)-\mathrm{F}(4)$ & $107.6(2)$ \\
\hline $\mathrm{C}(38)-\mathrm{C}(37)-\mathrm{S}(4)$ & $109.28(16)$ & $\mathrm{F}(5)-\mathrm{C}(48)-\mathrm{F}(4)$ & $102.6(2)$ \\
\hline $\mathrm{C}(40)-\mathrm{C}(37)-\mathrm{S}(4)$ & $102.74(15)$ & $\mathrm{F}(6 \mathrm{~A})-\mathrm{C}(48)-\mathrm{C}(45)$ & $120.2(10)$ \\
\hline $\mathrm{C}(37)-\mathrm{C}(38)-\mathrm{H}(38 \mathrm{~A})$ & 109.5 & $\mathrm{~F}(4 \mathrm{~A})-\mathrm{C}(48)-\mathrm{C}(45)$ & $112.2(8)$ \\
\hline $\mathrm{C}(37)-\mathrm{C}(38)-\mathrm{H}(38 \mathrm{~B})$ & 109.5 & $\mathrm{~F}(5 \mathrm{~A})-\mathrm{C}(48)-\mathrm{C}(45)$ & $110.5(9)$ \\
\hline
\end{tabular}




\begin{tabular}{|c|c|c|c|}
\hline $\mathrm{F}(6)-\mathrm{C}(48)-\mathrm{C}(45)$ & $114.57(19)$ & $\mathrm{F}(16)-\mathrm{C}(64)-\mathrm{F}(18)$ & $106.07(16)$ \\
\hline $\mathrm{F}(5)-\mathrm{C}(48)-\mathrm{C}(45)$ & $112.62(19)$ & $\mathrm{F}(17)-\mathrm{C}(64)-\mathrm{F}(18)$ & $105.49(16)$ \\
\hline $\mathrm{F}(4)-\mathrm{C}(48)-\mathrm{C}(45)$ & $111.8(2)$ & $\mathrm{F}(16)-\mathrm{C}(64)-\mathrm{C}(61)$ & $113.32(17)$ \\
\hline $\mathrm{C}(50)-\mathrm{C}(49)-\mathrm{C}(54)$ & $115.57(18)$ & $\mathrm{F}(17)-\mathrm{C}(64)-\mathrm{C}(61)$ & $111.99(17)$ \\
\hline $\mathrm{C}(50)-\mathrm{C}(49)-\mathrm{B}(1)$ & $121.59(17)$ & $\mathrm{F}(18)-\mathrm{C}(64)-\mathrm{C}(61)$ & $112.22(16)$ \\
\hline $\mathrm{C}(54)-\mathrm{C}(49)-\mathrm{B}(1)$ & $122.77(17)$ & $\mathrm{C}(70)-\mathrm{C}(65)-\mathrm{C}(66)$ & $115.06(18)$ \\
\hline $\mathrm{C}(51)-\mathrm{C}(50)-\mathrm{C}(49)$ & $122.53(19)$ & $\mathrm{C}(70)-\mathrm{C}(65)-\mathrm{B}(1)$ & $123.42(18)$ \\
\hline $\mathrm{C}(51)-\mathrm{C}(50)-\mathrm{H}(50)$ & 118.7 & $\mathrm{C}(66)-\mathrm{C}(65)-\mathrm{B}(1)$ & $121.28(18)$ \\
\hline $\mathrm{C}(49)-\mathrm{C}(50)-\mathrm{H}(50)$ & 118.7 & $\mathrm{C}(67)-\mathrm{C}(66)-\mathrm{C}(65)$ & $122.64(19)$ \\
\hline $\mathrm{C}(52)-\mathrm{C}(51)-\mathrm{C}(50)$ & $120.46(19)$ & $\mathrm{C}(67)-\mathrm{C}(66)-\mathrm{H}(66)$ & 118.7 \\
\hline $\mathrm{C}(52)-\mathrm{C}(51)-\mathrm{C}(55)$ & $120.59(19)$ & $\mathrm{C}(65)-\mathrm{C}(66)-\mathrm{H}(66)$ & 118.7 \\
\hline $\mathrm{C}(50)-\mathrm{C}(51)-\mathrm{C}(55)$ & $118.90(19)$ & $\mathrm{C}(68)-\mathrm{C}(67)-\mathrm{C}(66)$ & 120.91(19) \\
\hline $\mathrm{C}(51)-\mathrm{C}(52)-\mathrm{C}(53)$ & $118.58(19)$ & $\mathrm{C}(68)-\mathrm{C}(67)-\mathrm{C}(71)$ & $120.80(19)$ \\
\hline $\mathrm{C}(51)-\mathrm{C}(52)-\mathrm{H}(52)$ & 120.7 & $\mathrm{C}(66)-\mathrm{C}(67)-\mathrm{C}(71)$ & $118.28(19)$ \\
\hline $\mathrm{C}(53)-\mathrm{C}(52)-\mathrm{H}(52)$ & 120.7 & $\mathrm{C}(67)-\mathrm{C}(68)-\mathrm{C}(69)$ & $118.02(19)$ \\
\hline $\mathrm{C}(52)-\mathrm{C}(53)-\mathrm{C}(54)$ & $120.89(19)$ & $\mathrm{C}(67)-\mathrm{C}(68)-\mathrm{H}(68)$ & 121.0 \\
\hline $\mathrm{C}(52)-\mathrm{C}(53)-\mathrm{C}(56)$ & $118.34(19)$ & $\mathrm{C}(69)-\mathrm{C}(68)-\mathrm{H}(68)$ & 121.0 \\
\hline $\mathrm{C}(54)-\mathrm{C}(53)-\mathrm{C}(56)$ & $120.77(19)$ & $\mathrm{C}(68)-\mathrm{C}(69)-\mathrm{C}(70)$ & $120.71(19)$ \\
\hline $\mathrm{C}(53)-\mathrm{C}(54)-\mathrm{C}(49)$ & $121.94(19)$ & $\mathrm{C}(68)-\mathrm{C}(69)-\mathrm{C}(72)$ & $120.33(19)$ \\
\hline $\mathrm{C}(53)-\mathrm{C}(54)-\mathrm{H}(54)$ & 119.0 & $\mathrm{C}(70)-\mathrm{C}(69)-\mathrm{C}(72)$ & 118.94(19) \\
\hline $\mathrm{C}(49)-\mathrm{C}(54)-\mathrm{H}(54)$ & 119.0 & $\mathrm{C}(69)-\mathrm{C}(70)-\mathrm{C}(65)$ & $122.61(19)$ \\
\hline $\mathrm{F}(9)-\mathrm{C}(55)-\mathrm{F}(8)$ & $109.0(2)$ & $\mathrm{C}(69)-\mathrm{C}(70)-\mathrm{H}(70)$ & 118.7 \\
\hline $\mathrm{F}(9)-\mathrm{C}(55)-\mathrm{F}(7)$ & $104.7(2)$ & $\mathrm{C}(65)-\mathrm{C}(70)-\mathrm{H}(70)$ & 118.7 \\
\hline $\mathrm{F}(8)-\mathrm{C}(55)-\mathrm{F}(7)$ & $103.4(2)$ & $\mathrm{F}(21)-\mathrm{C}(71)-\mathrm{F}(19)$ & $106.6(2)$ \\
\hline $\mathrm{F}(9)-\mathrm{C}(55)-\mathrm{C}(51)$ & $113.8(2)$ & $\mathrm{F}(21)-\mathrm{C}(71)-\mathrm{F}(20)$ & $106.43(19)$ \\
\hline $\mathrm{F}(8)-\mathrm{C}(55)-\mathrm{C}(51)$ & $113.7(2)$ & $\mathrm{F}(19)-\mathrm{C}(71)-\mathrm{F}(20)$ & $105.7(2)$ \\
\hline $\mathrm{F}(7)-\mathrm{C}(55)-\mathrm{C}(51)$ & $111.4(2)$ & $\mathrm{F}(21)-\mathrm{C}(71)-\mathrm{C}(67)$ & $112.92(19)$ \\
\hline $\mathrm{F}(11)-\mathrm{C}(56)-\mathrm{F}(12)$ & $107.5(2)$ & $\mathrm{F}(19)-\mathrm{C}(71)-\mathrm{C}(67)$ & $112.57(19)$ \\
\hline $\mathrm{F}(11)-\mathrm{C}(56)-\mathrm{F}(10)$ & $105.90(19)$ & $\mathrm{F}(20)-\mathrm{C}(71)-\mathrm{C}(67)$ & $112.16(19)$ \\
\hline $\mathrm{F}(12)-\mathrm{C}(56)-\mathrm{F}(10)$ & $105.07(18)$ & $\mathrm{F}(23)-\mathrm{C}(72)-\mathrm{F}(24)$ & $106.26(19)$ \\
\hline $\mathrm{F}(11)-\mathrm{C}(56)-\mathrm{C}(53)$ & $111.83(19)$ & $\mathrm{F}(23)-\mathrm{C}(72)-\mathrm{F}(22)$ & $106.36(18)$ \\
\hline $\mathrm{F}(12)-\mathrm{C}(56)-\mathrm{C}(53)$ & $113.84(18)$ & $\mathrm{F}(24)-\mathrm{C}(72)-\mathrm{F}(22)$ & $105.86(17)$ \\
\hline $\mathrm{F}(10)-\mathrm{C}(56)-\mathrm{C}(53)$ & $112.12(19)$ & $\mathrm{F}(23)-\mathrm{C}(72)-\mathrm{C}(69)$ & $112.54(18)$ \\
\hline $\mathrm{C}(58)-\mathrm{C}(57)-\mathrm{C}(62)$ & $115.34(18)$ & $\mathrm{F}(24)-\mathrm{C}(72)-\mathrm{C}(69)$ & $112.81(17)$ \\
\hline $\mathrm{C}(58)-\mathrm{C}(57)-\mathrm{B}(1)$ & $124.25(17)$ & $\mathrm{F}(22)-\mathrm{C}(72)-\mathrm{C}(69)$ & $112.48(19)$ \\
\hline $\mathrm{C}(62)-\mathrm{C}(57)-\mathrm{B}(1)$ & $120.41(17)$ & $\mathrm{O}-\mathrm{C}(73)-\mathrm{C}(75)$ & $124.3(11)$ \\
\hline $\mathrm{C}(57)-\mathrm{C}(58)-\mathrm{C}(59)$ & $122.31(18)$ & $\mathrm{O}-\mathrm{C}(73)-\mathrm{H}(73 \mathrm{~A})$ & 106.3 \\
\hline $\mathrm{C}(57)-\mathrm{C}(58)-\mathrm{H}(58)$ & 118.8 & $\mathrm{C}(75)-\mathrm{C}(73)-\mathrm{H}(73 \mathrm{~A})$ & 106.3 \\
\hline $\mathrm{C}(59)-\mathrm{C}(58)-\mathrm{H}(58)$ & 118.8 & $\mathrm{O}-\mathrm{C}(73)-\mathrm{H}(73 \mathrm{~B})$ & 106.3 \\
\hline $\mathrm{C}(60)-\mathrm{C}(59)-\mathrm{C}(58)$ & $121.23(18)$ & $\mathrm{C}(75)-\mathrm{C}(73)-\mathrm{H}(73 \mathrm{~B})$ & 106.3 \\
\hline $\mathrm{C}(60)-\mathrm{C}(59)-\mathrm{C}(63)$ & $119.14(18)$ & H(73A)-C(73)-H(73B) & 106.4 \\
\hline $\mathrm{C}(58)-\mathrm{C}(59)-\mathrm{C}(63)$ & $119.58(18)$ & $\mathrm{C}(75)-\mathrm{C}(74)-\mathrm{O}$ & $119.3(5)$ \\
\hline$C(61)-C(60)-C(59)$ & $117.67(19)$ & $\mathrm{C}(75)-\mathrm{C}(74)-\mathrm{H}(74 \mathrm{~A})$ & 107.5 \\
\hline $\mathrm{C}(61)-\mathrm{C}(60)-\mathrm{H}(60)$ & 121.2 & $\mathrm{O}-\mathrm{C}(74)-\mathrm{H}(74 \mathrm{~A})$ & 107.5 \\
\hline $\mathrm{C}(59)-\mathrm{C}(60)-\mathrm{H}(60)$ & 121.2 & $\mathrm{C}(75)-\mathrm{C}(74)-\mathrm{H}(74 \mathrm{~B})$ & 107.5 \\
\hline $\mathrm{C}(60)-\mathrm{C}(61)-\mathrm{C}(62)$ & $120.89(19)$ & $\mathrm{O}-\mathrm{C}(74)-\mathrm{H}(74 \mathrm{~B})$ & 107.5 \\
\hline $\mathrm{C}(60)-\mathrm{C}(61)-\mathrm{C}(64)$ & $120.82(18)$ & $\mathrm{H}(74 \mathrm{~A})-\mathrm{C}(74)-\mathrm{H}(74 \mathrm{~B})$ & 107.0 \\
\hline $\mathrm{C}(62)-\mathrm{C}(61)-\mathrm{C}(64)$ & $118.28(18)$ & $\mathrm{C}(75)-\mathrm{C}(74)-\mathrm{H}(75 \mathrm{E})$ & 40.2 \\
\hline $\mathrm{C}(61)-\mathrm{C}(62)-\mathrm{C}(57)$ & $122.55(19)$ & $\mathrm{O}-\mathrm{C}(74)-\mathrm{H}(75 \mathrm{E})$ & 127.3 \\
\hline $\mathrm{C}(61)-\mathrm{C}(62)-\mathrm{H}(62)$ & 118.7 & H(74A)-C(74)-H(75E) & 67.7 \\
\hline $\mathrm{C}(57)-\mathrm{C}(62)-\mathrm{H}(62)$ & 118.7 & $\mathrm{H}(74 \mathrm{~B})-\mathrm{C}(74)-\mathrm{H}(75 \mathrm{E})$ & 124.4 \\
\hline $\mathrm{F}(13)-\mathrm{C}(63)-\mathrm{F}(14)$ & $105.45(18)$ & $\mathrm{C}(75)-\mathrm{C}(74)-\mathrm{H}(75 \mathrm{~F})$ & 40.2 \\
\hline $\mathrm{F}(13)-\mathrm{C}(63)-\mathrm{F}(15)$ & 106.71(18) & $\mathrm{O}-\mathrm{C}(74)-\mathrm{H}(75 \mathrm{~F})$ & 139.7 \\
\hline $\mathrm{F}(14)-\mathrm{C}(63)-\mathrm{F}(15)$ & $105.84(18)$ & H(74A)-C(74)-H(75F) & 112.0 \\
\hline $\mathrm{F}(13)-\mathrm{C}(63)-\mathrm{C}(59)$ & $112.62(18)$ & H(74B)-C(74)-H(75F) & 68.1 \\
\hline $\mathrm{F}(14)-\mathrm{C}(63)-\mathrm{C}(59)$ & $112.64(17)$ & $\mathrm{H}(75 \mathrm{E})-\mathrm{C}(74)-\mathrm{H}(75 \mathrm{~F})$ & 64.6 \\
\hline $\mathrm{F}(15)-\mathrm{C}(63)-\mathrm{C}(59)$ & $113.00(18)$ & $\mathrm{C}(74)-\mathrm{C}(75)-\mathrm{C}(73)$ & $56.2(6)$ \\
\hline$F(16)-C(64)-F(17)$ & 107.21(16) & $\mathrm{C}(74)-\mathrm{C}(75)-\mathrm{H}(75 \mathrm{~A})$ & 114.0 \\
\hline
\end{tabular}


$\mathrm{C}(73)-\mathrm{C}(75)-\mathrm{H}(75 \mathrm{~A})$

$\mathrm{C}(74)-\mathrm{C}(75)-\mathrm{H}(75 \mathrm{~B})$

$\mathrm{C}(73)-\mathrm{C}(75)-\mathrm{H}(75 \mathrm{~B})$

$\mathrm{H}(75 \mathrm{~A})-\mathrm{C}(75)-\mathrm{H}(75 \mathrm{~B})$

$\mathrm{C}(74)-\mathrm{C}(75)-\mathrm{H}(75 \mathrm{C})$

$\mathrm{C}(73)-\mathrm{C}(75)-\mathrm{H}(75 \mathrm{C})$

$\mathrm{H}(75 \mathrm{~A})-\mathrm{C}(75)-\mathrm{H}(75 \mathrm{C})$

$\mathrm{H}(75 \mathrm{~B})-\mathrm{C}(75)-\mathrm{H}(75 \mathrm{C})$

$\mathrm{C}(74)-\mathrm{C}(75)-\mathrm{H}(75 \mathrm{D})$

$\mathrm{C}(73)-\mathrm{C}(75)-\mathrm{H}(75 \mathrm{D})$

$\mathrm{H}(75 \mathrm{~A})-\mathrm{C}(75)-\mathrm{H}(75 \mathrm{D})$

$\mathrm{H}(75 \mathrm{~B})-\mathrm{C}(75)-\mathrm{H}(75 \mathrm{D})$

$\mathrm{H}(75 \mathrm{C})-\mathrm{C}(75)-\mathrm{H}(75 \mathrm{D})$
167.4

114.3

75.9

104.0

114.1

88.1

103.9

105.4

167.9

111.7

77.9

58.5

62.4
$\mathrm{C}(74)-\mathrm{C}(75)-\mathrm{H}(75 \mathrm{E})$

$\mathrm{C}(73)-\mathrm{C}(75)-\mathrm{H}(75 \mathrm{E})$

$\mathrm{H}(75 \mathrm{~A})-\mathrm{C}(75)-\mathrm{H}(75 \mathrm{E})$

$\mathrm{H}(75 \mathrm{~B})-\mathrm{C}(75)-\mathrm{H}(75 \mathrm{E})$

$\mathrm{H}(75 \mathrm{C})-\mathrm{C}(75)-\mathrm{H}(75 \mathrm{E})$

$\mathrm{H}(75 \mathrm{D})-\mathrm{C}(75)-\mathrm{H}(75 \mathrm{E})$

$\mathrm{C}(74)-\mathrm{C}(75)-\mathrm{H}(75 \mathrm{~F})$

$\mathrm{C}(73)-\mathrm{C}(75)-\mathrm{H}(75 \mathrm{~F})$

$\mathrm{H}(75 \mathrm{~A})-\mathrm{C}(75)-\mathrm{H}(75 \mathrm{~F})$

$\mathrm{H}(75 \mathrm{~B})-\mathrm{C}(75)-\mathrm{H}(75 \mathrm{~F})$

$\mathrm{H}(75 \mathrm{C})-\mathrm{C}(75)-\mathrm{H}(75 \mathrm{~F})$

$\mathrm{H}(75 \mathrm{D})-\mathrm{C}(75)-\mathrm{H}(75 \mathrm{~F})$

$\mathrm{H}(75 \mathrm{E})-\mathrm{C}(75)-\mathrm{H}(75 \mathrm{~F})$
82.9

112.8

71.0

162.1

60.8

103.7

83.1

113.7

54.7

77.7

157.8

103.6

110.6

Symmetry transformations used to generate equivalent atoms:

$\# 1-\mathrm{x}+2,-\mathrm{y}+1,-\mathrm{z}^{+1}$ 
Table 9. Anisotropic displacement parameters $\left(\AA^{2} \times 10^{3}\right)$ for 3. The anisotropic displacement factor exponent takes the form: $-2 \pi^{2}\left[h^{2} a^{* 2} U^{11}+\ldots+2 h \mathrm{k} \mathrm{a}^{*} b^{*} U^{12}\right]$

\begin{tabular}{|c|c|c|c|c|c|c|}
\hline & $\mathrm{U}^{11}$ & $\mathrm{U}^{22}$ & $\mathrm{U}^{33}$ & $\mathrm{U}^{23}$ & $\mathrm{U}^{13}$ & $\mathrm{U}^{12}$ \\
\hline $\mathrm{Cu}(1)$ & $15(1)$ & $20(1)$ & $16(1)$ & $-3(1)$ & $-2(1)$ & $-2(1)$ \\
\hline $\mathrm{Cu}(2)$ & $16(1)$ & $19(1)$ & 21(1) & $-5(1)$ & $-4(1)$ & $0(1)$ \\
\hline $\mathrm{N}(1)$ & $15(1)$ & $16(1)$ & 19(1) & $-3(1)$ & $-2(1)$ & $-1(1)$ \\
\hline $\mathrm{N}(2)$ & $14(1)$ & $20(1)$ & $16(1)$ & $-2(1)$ & $-1(1)$ & $-1(1)$ \\
\hline $\mathrm{S}(1)$ & $15(1)$ & $17(1)$ & $33(1)$ & $-2(1)$ & $-3(1)$ & $-2(1)$ \\
\hline $\mathrm{S}(2)$ & $20(1)$ & $19(1)$ & 17(1) & $-6(1)$ & $2(1)$ & $-4(1)$ \\
\hline $\mathrm{S}(3)$ & 14(1) & $32(1)$ & 17(1) & $-1(1)$ & $-2(1)$ & $-2(1)$ \\
\hline $\mathrm{S}(4)$ & $23(1)$ & $24(1)$ & 19(1) & $-9(1)$ & $-7(1)$ & $5(1)$ \\
\hline $\mathrm{O}$ & $146(7)$ & $99(5)$ & $302(13)$ & $82(7)$ & $-145(9)$ & $-79(5)$ \\
\hline $\mathrm{B}(1)$ & $16(1)$ & $18(1)$ & 13(1) & $-4(1)$ & $-2(1)$ & 1(1) \\
\hline $\mathrm{C}(1)$ & $12(1)$ & $23(1)$ & $20(1)$ & $-3(1)$ & $-5(1)$ & $-3(1)$ \\
\hline$C(2)$ & $12(1)$ & $24(1)$ & $27(1)$ & $-2(1)$ & $-4(1)$ & $-3(1)$ \\
\hline$C(3)$ & $20(1)$ & $25(1)$ & 31(1) & $6(1)$ & $-5(1)$ & $-5(1)$ \\
\hline $\mathrm{C}(4)$ & $23(1)$ & $39(1)$ & $22(1)$ & $2(1)$ & $-2(1)$ & $-10(1)$ \\
\hline$C(5)$ & $18(1)$ & $38(1)$ & $22(1)$ & $-10(1)$ & $-2(1)$ & $-7(1)$ \\
\hline$C(6)$ & $17(1)$ & $24(1)$ & 23(1) & $-6(1)$ & $-5(1)$ & $-4(1)$ \\
\hline$C(7)$ & $22(1)$ & $18(1)$ & 13(1) & $-7(1)$ & $-1(1)$ & $2(1)$ \\
\hline $\mathrm{C}(8)$ & $21(1)$ & $21(1)$ & $14(1)$ & $-8(1)$ & $-1(1)$ & 1(1) \\
\hline$C(9)$ & $33(1)$ & $22(1)$ & $16(1)$ & $-6(1)$ & $3(1)$ & $-1(1)$ \\
\hline$C(10)$ & $42(1)$ & $22(1)$ & 17(1) & $-7(1)$ & $-9(1)$ & $9(1)$ \\
\hline $\mathrm{C}(11)$ & $28(1)$ & $26(1)$ & $28(1)$ & $-14(1)$ & $-12(1)$ & $9(1)$ \\
\hline$C(12)$ & $21(1)$ & $22(1)$ & $25(1)$ & $-11(1)$ & $-5(1)$ & $0(1)$ \\
\hline$C(13)$ & $12(1)$ & $19(1)$ & $24(1)$ & $-2(1)$ & $-5(1)$ & $-3(1)$ \\
\hline$C(14)$ & 13(1) & $27(1)$ & 24(1) & $-2(1)$ & $-4(1)$ & $-4(1)$ \\
\hline$C(15)$ & $21(1)$ & $28(1)$ & 28(1) & $6(1)$ & $-6(1)$ & $-4(1)$ \\
\hline$C(16)$ & $28(1)$ & $23(1)$ & $45(2)$ & 1(1) & $-14(1)$ & 1(1) \\
\hline$C(17)$ & $21(1)$ & $24(1)$ & $47(2)$ & $-12(1)$ & $-10(1)$ & $5(1)$ \\
\hline$C(18)$ & $18(1)$ & $26(1)$ & 27(1) & $-6(1)$ & $-5(1)$ & $-1(1)$ \\
\hline$C(19)$ & $23(1)$ & $16(1)$ & 17(1) & $-8(1)$ & $0(1)$ & $-1(1)$ \\
\hline$C(20)$ & $24(1)$ & $20(1)$ & $17(1)$ & $-9(1)$ & $-2(1)$ & 1(1) \\
\hline$C(21)$ & $37(1)$ & $21(1)$ & $15(1)$ & $-4(1)$ & $-2(1)$ & $5(1)$ \\
\hline$C(22)$ & $38(1)$ & $22(1)$ & 20(1) & $-6(1)$ & $10(1)$ & $-6(1)$ \\
\hline$C(23)$ & $26(1)$ & $28(1)$ & $25(1)$ & $-13(1)$ & $7(1)$ & $-8(1)$ \\
\hline$C(24)$ & $20(1)$ & $24(1)$ & $20(1)$ & $-9(1)$ & $-1(1)$ & $0(1)$ \\
\hline$C(25)$ & $19(1)$ & $26(1)$ & $48(2)$ & $-12(1)$ & $-5(1)$ & $-8(1)$ \\
\hline$C(26)$ & $34(1)$ & $35(2)$ & $74(2)$ & $-28(2)$ & $-10(1)$ & $-5(1)$ \\
\hline$C(27)$ & $28(1)$ & $38(2)$ & 61(2) & $0(1)$ & $-8(1)$ & $-18(1)$ \\
\hline$C(28)$ & $20(1)$ & $32(1)$ & $42(2)$ & $-13(1)$ & $-10(1)$ & $0(1)$ \\
\hline$C(29)$ & 33(1) & $21(1)$ & $20(1)$ & $-9(1)$ & 1(1) & $-8(1)$ \\
\hline $\mathrm{C}(30)$ & $36(1)$ & $38(2)$ & $37(2)$ & $-18(1)$ & $-2(1)$ & $-14(1)$ \\
\hline$C(31)$ & $38(1)$ & $29(1)$ & 20(1) & $-11(1)$ & $2(1)$ & $-4(1)$ \\
\hline$C(32)$ & $53(2)$ & $22(1)$ & $28(1)$ & $-10(1)$ & 1(1) & $-7(1)$ \\
\hline$C(33)$ & 14(1) & $44(1)$ & 18(1) & $-7(1)$ & $-4(1)$ & $-2(1)$ \\
\hline$C(34)$ & 18(1) & $47(2)$ & $26(1)$ & $-13(1)$ & $-2(1)$ & $-9(1)$ \\
\hline$C(35)$ & $21(1)$ & $57(2)$ & $29(1)$ & $-4(1)$ & $-9(1)$ & 1(1) \\
\hline$C(36)$ & $26(1)$ & $55(2)$ & $26(1)$ & $-17(1)$ & $-6(1)$ & 1(1) \\
\hline$C(37)$ & 31(1) & $35(1)$ & 29(1) & $-21(1)$ & $-12(1)$ & $6(1)$ \\
\hline $\mathrm{C}(38)$ & $56(2)$ & $47(2)$ & $22(1)$ & $-18(1)$ & $-11(1)$ & $11(1)$ \\
\hline$C(39)$ & $34(1)$ & $31(1)$ & $35(1)$ & $-19(1)$ & $-7(1)$ & $3(1)$ \\
\hline $\mathrm{C}(40)$ & $37(2)$ & $52(2)$ & $53(2)$ & $-33(1)$ & $-22(1)$ & $9(1)$ \\
\hline C(41) & $19(1)$ & $17(1)$ & 13(1) & 1(1) & $-3(1)$ & $0(1)$ \\
\hline
\end{tabular}




\begin{tabular}{|c|c|c|c|c|c|c|}
\hline$C(42)$ & $18(1)$ & $17(1)$ & $16(1)$ & $-1(1)$ & $0(1)$ & $0(1)$ \\
\hline C(43) & $23(1)$ & $16(1)$ & $20(1)$ & $-4(1)$ & $-2(1)$ & $-2(1)$ \\
\hline $\mathrm{C}(44)$ & $25(1)$ & $22(1)$ & $18(1)$ & $-8(1)$ & $0(1)$ & $2(1)$ \\
\hline$C(45)$ & $18(1)$ & $25(1)$ & $19(1)$ & $-5(1)$ & $0(1)$ & $2(1)$ \\
\hline $\mathrm{C}(46)$ & $18(1)$ & $22(1)$ & $16(1)$ & $-5(1)$ & $-3(1)$ & $-1(1)$ \\
\hline $\mathrm{C}(47)$ & $25(1)$ & $25(1)$ & $31(1)$ & $-13(1)$ & $2(1)$ & $-2(1)$ \\
\hline C(48) & $22(1)$ & $38(1)$ & $31(1)$ & $-17(1)$ & $-1(1)$ & $3(1)$ \\
\hline C(49) & $17(1)$ & $15(1)$ & $18(1)$ & $-4(1)$ & $-2(1)$ & $-3(1)$ \\
\hline $\mathrm{C}(50)$ & $19(1)$ & 19(1) & $19(1)$ & $-4(1)$ & $-2(1)$ & $0(1)$ \\
\hline $\mathrm{C}(51)$ & $22(1)$ & $25(1)$ & $18(1)$ & $-6(1)$ & 1(1) & $-2(1)$ \\
\hline$C(52)$ & $26(1)$ & $22(1)$ & $14(1)$ & 1(1) & $-3(1)$ & $-4(1)$ \\
\hline$C(53)$ & $22(1)$ & $16(1)$ & $19(1)$ & $-2(1)$ & $-3(1)$ & $-3(1)$ \\
\hline$C(54)$ & $18(1)$ & $17(1)$ & $19(1)$ & $-5(1)$ & 1(1) & $-1(1)$ \\
\hline$C(55)$ & $34(1)$ & $50(2)$ & 21(1) & $-10(1)$ & $0(1)$ & $9(1)$ \\
\hline$C(56)$ & $40(1)$ & $25(1)$ & $23(1)$ & $-4(1)$ & $-9(1)$ & $9(1)$ \\
\hline $\mathrm{C}(57)$ & $13(1)$ & 18(1) & $17(1)$ & $-4(1)$ & $-3(1)$ & $4(1)$ \\
\hline $\mathrm{C}(58)$ & $17(1)$ & $19(1)$ & $14(1)$ & $-3(1)$ & $-2(1)$ & $4(1)$ \\
\hline $\mathrm{C}(59)$ & $16(1)$ & 19(1) & $20(1)$ & $-9(1)$ & $-4(1)$ & $3(1)$ \\
\hline $\mathrm{C}(60)$ & $14(1)$ & $17(1)$ & $23(1)$ & $-5(1)$ & $-1(1)$ & $0(1)$ \\
\hline $\mathrm{C}(61)$ & $14(1)$ & $20(1)$ & $16(1)$ & $-4(1)$ & $-2(1)$ & $4(1)$ \\
\hline $\mathrm{C}(62)$ & $16(1)$ & $20(1)$ & $18(1)$ & $-7(1)$ & $-4(1)$ & 1(1) \\
\hline$C(63)$ & $25(1)$ & $27(1)$ & $23(1)$ & $-10(1)$ & $-3(1)$ & $-2(1)$ \\
\hline $\mathrm{C}(64)$ & $20(1)$ & $23(1)$ & $20(1)$ & $-5(1)$ & $-2(1)$ & $-1(1)$ \\
\hline$C(65)$ & 19(1) & 19(1) & $16(1)$ & $-8(1)$ & $-1(1)$ & $0(1)$ \\
\hline$C(66)$ & $23(1)$ & $18(1)$ & $18(1)$ & $-6(1)$ & $-3(1)$ & 2(1) \\
\hline C(67) & $18(1)$ & $24(1)$ & 23(1) & $-10(1)$ & $0(1)$ & $-2(1)$ \\
\hline$C(68)$ & $16(1)$ & $27(1)$ & $27(1)$ & $-12(1)$ & $-5(1)$ & $4(1)$ \\
\hline $\mathrm{C}(69)$ & $23(1)$ & 21(1) & $20(1)$ & $-9(1)$ & $-5(1)$ & $4(1)$ \\
\hline$C(70)$ & $19(1)$ & 19(1) & 19(1) & $-7(1)$ & $-1(1)$ & $0(1)$ \\
\hline $\mathrm{C}(71)$ & $22(1)$ & $36(1)$ & $31(1)$ & $-7(1)$ & $0(1)$ & $-4(1)$ \\
\hline$C(72)$ & $26(1)$ & $30(1)$ & $27(1)$ & $-8(1)$ & $-8(1)$ & $6(1)$ \\
\hline$C(73)$ & $59(6)$ & $48(5)$ & $130(11)$ & $9(6)$ & $-8(6)$ & $28(4)$ \\
\hline$C(74)$ & $34(3)$ & $40(4)$ & $69(4)$ & $-19(3)$ & $-10(3)$ & $-1(3)$ \\
\hline$C(75)$ & $87(4)$ & $64(3)$ & $227(7)$ & $35(4)$ & $-59(4)$ & $-22(3)$ \\
\hline $\mathrm{F}(1)$ & $25(1)$ & $59(1)$ & $75(1)$ & $-47(1)$ & $10(1)$ & $-14(1)$ \\
\hline $\mathrm{F}(2)$ & $51(1)$ & 21(1) & $57(1)$ & $-9(1)$ & $-16(1)$ & $-6(1)$ \\
\hline $\mathrm{F}(3)$ & $48(1)$ & $44(1)$ & $33(1)$ & $-10(1)$ & $-12(1)$ & $-15(1)$ \\
\hline $\mathrm{F}(4)$ & $34(1)$ & $69(2)$ & $93(2)$ & $24(1)$ & $12(1)$ & $29(1)$ \\
\hline $\mathrm{F}(5)$ & $28(1)$ & $105(2)$ & $28(1)$ & $-17(1)$ & $10(1)$ & $-1(1)$ \\
\hline $\mathrm{F}(6)$ & $19(1)$ & $148(3)$ & $165(3)$ & $-140(2)$ & 21(1) & $-24(1)$ \\
\hline $\mathrm{F}(7)$ & $59(1)$ & $62(1)$ & $59(1)$ & $-42(1)$ & $3(1)$ & $10(1)$ \\
\hline $\mathrm{F}(8)$ & $25(1)$ & $112(2)$ & $47(1)$ & $-39(1)$ & $5(1)$ & $12(1)$ \\
\hline $\mathrm{F}(9)$ & $98(1)$ & $88(1)$ & $17(1)$ & $-4(1)$ & $12(1)$ & $44(1)$ \\
\hline $\mathrm{F}(10)$ & $72(1)$ & $22(1)$ & $37(1)$ & $8(1)$ & 11(1) & $13(1)$ \\
\hline $\mathrm{F}(11)$ & $72(1)$ & $62(1)$ & $84(1)$ & $-42(1)$ & $-60(1)$ & $38(1)$ \\
\hline $\mathrm{F}(12)$ & $36(1)$ & $43(1)$ & $32(1)$ & $0(1)$ & $1(1)$ & $21(1)$ \\
\hline $\mathrm{F}(13)$ & $39(1)$ & $73(1)$ & $32(1)$ & $-36(1)$ & $7(1)$ & $-17(1)$ \\
\hline$F(14)$ & $29(1)$ & $56(1)$ & $43(1)$ & $-26(1)$ & $-17(1)$ & $5(1)$ \\
\hline $\mathrm{F}(15)$ & $85(1)$ & $26(1)$ & $43(1)$ & $-10(1)$ & $-26(1)$ & $-16(1)$ \\
\hline $\mathrm{F}(16)$ & $38(1)$ & $38(1)$ & $21(1)$ & $1(1)$ & $0(1)$ & $-20(1)$ \\
\hline $\mathrm{F}(17)$ & $39(1)$ & $34(1)$ & $19(1)$ & $-6(1)$ & $5(1)$ & $9(1)$ \\
\hline $\mathrm{F}(18)$ & $25(1)$ & $33(1)$ & $19(1)$ & $-2(1)$ & $-8(1)$ & 1(1) \\
\hline $\mathrm{F}(19)$ & $59(1)$ & $36(1)$ & $90(1)$ & $-22(1)$ & $29(1)$ & $-22(1)$ \\
\hline $\mathrm{F}(20)$ & $47(1)$ & $83(1)$ & $33(1)$ & $-7(1)$ & $8(1)$ & $-30(1)$ \\
\hline $\mathrm{F}(21)$ & $17(1)$ & $69(1)$ & 78(1) & $15(1)$ & $-9(1)$ & $-11(1)$ \\
\hline $\mathrm{F}(22)$ & $23(1)$ & $50(1)$ & $33(1)$ & 1(1) & $-13(1)$ & $5(1)$ \\
\hline
\end{tabular}


$\mathrm{F}(23) \quad 96(1)$

$\mathrm{F}(24) \quad 28(1)$

25(1)

41(1)

42(1)

$34(1)$

$-11(1)$

10(1)

$-30(1)$

$-6(1)$

21(1)

$0(1)$ 
Table 10. Hydrogen coordinates ( $\left.\mathrm{x} 10^{4}\right)$ and isotropic displacement parameters $\left(\AA^{2} \times 10^{3}\right)$ for 3 .

\begin{tabular}{|c|c|c|c|c|}
\hline & $\mathrm{x}$ & $\mathrm{y}$ & $\mathrm{z}$ & $\mathrm{U}_{\mathrm{eq}}$ \\
\hline $\mathrm{H}(3)$ & 5006 & 6419 & 9023 & 33 \\
\hline $\mathrm{H}(4)$ & 3955 & 7094 & 9747 & 36 \\
\hline $\mathrm{H}(5)$ & 3659 & 8338 & 9309 & 30 \\
\hline $\mathrm{H}(6)$ & 4566 & 8910 & 8189 & 25 \\
\hline $\mathrm{H}(9)$ & 6646 & 10475 & 5758 & 29 \\
\hline $\mathrm{H}(10)$ & 4505 & 10574 & 5460 & 32 \\
\hline $\mathrm{H}(11)$ & 3020 & 9725 & 6097 & 31 \\
\hline $\mathrm{H}(12)$ & 3697 & 8738 & 6952 & 26 \\
\hline $\mathrm{H}(15)$ & 9469 & 5919 & 8676 & 34 \\
\hline $\mathrm{H}(16)$ & 10470 & 5123 & 8064 & 40 \\
\hline $\mathrm{H}(17)$ & 11017 & 5524 & 6854 & 36 \\
\hline $\mathrm{H}(18)$ & 10429 & 6690 & 6245 & 28 \\
\hline $\mathrm{H}(21)$ & 8942 & 9297 & 4681 & 30 \\
\hline $\mathrm{H}(22)$ & 11137 & 9519 & 4631 & 33 \\
\hline $\mathrm{H}(23)$ & 12420 & 8796 & 5466 & 31 \\
\hline $\mathrm{H}(24)$ & 11483 & 7919 & 6397 & 25 \\
\hline $\mathrm{H}(26 \mathrm{~A})$ & 4669 & 5757 & 6624 & 67 \\
\hline $\mathrm{H}(26 \mathrm{~B})$ & 5883 & 5551 & 7083 & 67 \\
\hline $\mathrm{H}(26 \mathrm{C})$ & 5901 & 6253 & 6435 & 67 \\
\hline $\mathrm{H}(27 \mathrm{~A})$ & 3486 & 6219 & 8229 & 66 \\
\hline $\mathrm{H}(27 \mathrm{~B})$ & 4498 & 5555 & 8206 & 66 \\
\hline $\mathrm{H}(27 \mathrm{C})$ & 3254 & 5686 & 7769 & 66 \\
\hline $\mathrm{H}(28 \mathrm{~A})$ & 4389 & 7300 & 6504 & 46 \\
\hline $\mathrm{H}(28 \mathrm{~B})$ & 3555 & 7321 & 7229 & 46 \\
\hline $\mathrm{H}(28 \mathrm{C})$ & 3122 & 6834 & 6769 & 46 \\
\hline $\mathrm{H}(30 \mathrm{~A})$ & 9574 & 10346 & 7819 & 52 \\
\hline $\mathrm{H}(30 \mathrm{~B})$ & 10069 & 10179 & 7084 & 52 \\
\hline $\mathrm{H}(30 \mathrm{C})$ & 9704 & 9535 & 7762 & 52 \\
\hline $\mathrm{H}(31 \mathrm{~A})$ & 7331 & 9362 & 8232 & 43 \\
\hline $\mathrm{H}(31 \mathrm{~B})$ & 6225 & 9885 & 7832 & 43 \\
\hline $\mathrm{H}(31 \mathrm{C})$ & 7132 & 10173 & 8287 & 43 \\
\hline $\mathrm{H}(32 \mathrm{~A})$ & 6846 & 10890 & 6798 & 51 \\
\hline $\mathrm{H}(32 \mathrm{~B})$ & 8350 & 10996 & 6504 & 51 \\
\hline $\mathrm{H}(32 \mathrm{C})$ & 7802 & 11186 & 7222 & 51 \\
\hline $\mathrm{H}(34 \mathrm{~A})$ & 10759 & 8406 & 7712 & 45 \\
\hline $\mathrm{H}(34 \mathrm{~B})$ & 11436 & 7628 & 7745 & 45 \\
\hline $\mathrm{H}(34 \mathrm{C})$ & 11941 & 8117 & 8183 & 45 \\
\hline $\mathrm{H}(35 \mathrm{~A})$ & 11522 & 7125 & 9332 & 55 \\
\hline $\mathrm{H}(35 \mathrm{~B})$ & 11166 & 6652 & 8840 & 55 \\
\hline $\mathrm{H}(35 \mathrm{C})$ & 10149 & 6751 & 9482 & 55 \\
\hline $\mathrm{H}(36 \mathrm{~A})$ & 9088 & 7944 & 9453 & 52 \\
\hline $\mathrm{H}(36 \mathrm{~B})$ & 9205 & 8583 & 8748 & 52 \\
\hline $\mathrm{H}(36 \mathrm{C})$ & 10411 & 8365 & 9213 & 52 \\
\hline $\mathrm{H}(38 \mathrm{~A})$ & 8860 & 8185 & 4264 & 60 \\
\hline $\mathrm{H}(38 \mathrm{~B})$ & 7451 & 8550 & 4092 & 60 \\
\hline $\mathrm{H}(38 \mathrm{C})$ & 7904 & 7808 & 3908 & 60 \\
\hline $\mathrm{H}(39 \mathrm{~A})$ & 8172 & 6679 & 4944 & 47 \\
\hline H(39B) & 7807 & 6760 & 5724 & 47 \\
\hline $\mathrm{H}(39 \mathrm{C})$ & 9078 & 7121 & 5262 & 47 \\
\hline $\mathrm{H}(40 \mathrm{~A})$ & 5900 & 7245 & 4610 & 64 \\
\hline
\end{tabular}




\begin{tabular}{lrrrr}
$\mathrm{H}(40 \mathrm{~B})$ & 5438 & 7982 & 4800 & 64 \\
$\mathrm{H}(40 \mathrm{C})$ & 5589 & 7259 & 5413 & 64 \\
$\mathrm{H}(42)$ & 2575 & 6388 & 1422 & 22 \\
$\mathrm{H}(44)$ & 5506 & 5418 & 422 & 26 \\
$\mathrm{H}(46)$ & 6300 & 6903 & 1386 & 23 \\
$\mathrm{H}(50)$ & 2609 & 7558 & 3177 & 23 \\
$\mathrm{H}(52)$ & 4431 & 6146 & 4705 & 26 \\
$\mathrm{H}(54)$ & 5507 & 6216 & 2667 & 22 \\
$\mathrm{H}(58)$ & 5195 & 8156 & 2592 & 21 \\
$\mathrm{H}(60)$ & 6991 & 9607 & 945 & 22 \\
$\mathrm{H}(62)$ & 4789 & 8109 & 619 & 21 \\
$\mathrm{H}(66)$ & 1522 & 6738 & 2562 & 24 \\
$\mathrm{H}(68)$ & -1193 & 8035 & 1460 & 27 \\
$\mathrm{H}(70)$ & 2627 & 8509 & 1059 & 22 \\
$\mathrm{H}(73 \mathrm{~A})$ & 9790 & 5592 & 5501 & 105 \\
$\mathrm{H}(73 \mathrm{~B})$ & 10597 & 4863 & 5788 & 105 \\
$\mathrm{H}(74 \mathrm{~A})$ & 8041 & 4583 & 5342 & 55 \\
$\mathrm{H}(74 \mathrm{~B})$ & 9129 & 4045 & 5730 & 55 \\
$\mathrm{H}(75 \mathrm{~A})$ & 7790 & 4454 & 6450 & 203 \\
$\mathrm{H}(75 \mathrm{~B})$ & 9198 & 4716 & 6431 & 203 \\
$\mathrm{H}(75 \mathrm{C})$ & 8124 & 5245 & 6048 & 203 \\
$\mathrm{H}(75 \mathrm{D})$ & 8375 & 4974 & 6552 & 203 \\
$\mathrm{H}(75 \mathrm{E})$ & 7684 & 4868 & 5929 & 203 \\
$\mathrm{H}(75 \mathrm{~F})$ & 8570 & 4244 & 6348 & 203 \\
& & & & \\
\hline
\end{tabular}


Table 11. Crystal data and structure refinement for 4.

\begin{tabular}{|c|c|}
\hline Name of Complex & $\mathrm{SNS}-\mathrm{CuCl}$ \\
\hline Empirical formula & $\mathrm{C}_{20} \mathrm{H}_{26} \mathrm{ClCuNS}_{2}$ \\
\hline Formula weight & 443.53 \\
\hline Temperature & $96 \mathrm{~K}$ \\
\hline Wavelength & $0.71073 \AA$ \\
\hline Crystal system & Orthorhombic \\
\hline Crystal habit & prism \\
\hline Crystal color & green \\
\hline Crystal size & $0.19 \times .19 \times .19 \mathrm{~mm}^{3}$ \\
\hline Space group & pbca $(\# 61)$ \\
\hline \multirow[t]{3}{*}{ Unit cell dimensions } & $\mathrm{a}=17.0807(13) \AA$ \\
\hline & $\mathrm{b}=12.3173(10) \AA$ \\
\hline & $c=19.5782(15) \AA$ \\
\hline Volume & $4119.0(6) \AA^{3}$ \\
\hline $\mathrm{Z}$ & 8 \\
\hline Density (calculated) & $1.430 \mathrm{Mg} / \mathrm{m}^{3}$ \\
\hline Absorption coefficient & $1.396 \mathrm{~mm}^{-1}$ \\
\hline $\mathrm{F}(000)$ & 1848 \\
\hline Theta range for data collection & 2.08 to $28.54^{\circ}$. \\
\hline Index ranges & $-22<=\mathrm{h}<=22,-12<=\mathrm{k}<=16,-21<=\mathrm{l}<=24$ \\
\hline Reflections collected & 24577 \\
\hline Independent reflections & $4901[\mathrm{R}(\mathrm{int})=0.0698]$ \\
\hline Completeness to theta $=28.54^{\circ}$ & $93.5 \%$ \\
\hline Absorption correction & None \\
\hline Refinement method & Full-matrix least-squares on $\mathrm{F}^{2}$ \\
\hline Data / restraints / parameters & $4901 / 0 / 232$ \\
\hline Goodness-of-fit on $\mathrm{F}^{2}$ & 1.235 \\
\hline Final $R$ indices $[\mathrm{I}>2 \operatorname{sigma}(\mathrm{I})]$ & $\mathrm{R} 1=0.0381, \mathrm{wR} 2=0.0694$ \\
\hline $\mathrm{R}$ indices (all data) & $\mathrm{R} 1=0.0664, \mathrm{wR} 2=0.0753$ \\
\hline Largest diff. peak and hole & 0.506 and -0.441 e. $\AA^{-3}$ \\
\hline
\end{tabular}

\section{Special Refinement Details}

Refinement of F2 against ALL reflections. The weighted R-factor $(w \mathrm{R})$ and goodness of fit $(\mathrm{S})$ are based on F2, conventional R-factors (R) are based on F, with F set to zero for negative F2. The threshold expression of F2 $>2 \int(\mathrm{F} 2)$ is used only for calculating R-factors(gt) etc. and is not relevant to the choice of reflections for refinement.

All esds (except the esd in the dihedral angle between two 1.s. planes) are estimated using the full covariance matrix. The cell esds are taken into account individually in the estimation of esds in distances, angles and torsion angles; correlations between esds in cell parameters are only used when they are defined by crystal symmetry. An approximate (isotropic) treatment of cell esds is used for estimating esds involving 1.s. planes. 
Table 12. Atomic coordinates $\left(\mathrm{x} 10^{4}\right)$ and equivalent isotropic displacement parameters $\left(\AA^{2} \times 10^{3}\right)$ for 4. $U_{\text {eq }}$ is defined as one third of the trace of the orthogonalized $U^{i j}$ tensor.

\begin{tabular}{lrrrr}
\hline & $\mathrm{x}$ & $\mathrm{y}$ & $\mathrm{z}$ & $\mathrm{U}_{\mathrm{eq}}$ \\
\hline $\mathrm{Cu}$ & & & & $12(1)$ \\
$\mathrm{N}$ & $947(1)$ & $3017(1)$ & $3636(1)$ & $14(1)$ \\
$\mathrm{S}(1)$ & $186(1)$ & $2256(2)$ & $4172(1)$ & $13(1)$ \\
$\mathrm{S}(2)$ & $1844(1)$ & $2552(1)$ & $4497(1)$ & $12(1)$ \\
$\mathrm{Cl}$ & $-111(1)$ & $3634(1)$ & $2978(1)$ & $17(1)$ \\
$\mathrm{C}(1)$ & $1818(1)$ & $3735(1)$ & $2938(1)$ & $12(1)$ \\
$\mathrm{C}(2)$ & $336(1)$ & $2040(2)$ & $4851(1)$ & $12(1)$ \\
$\mathrm{C}(3)$ & $1118(1)$ & $2140(2)$ & $5088(1)$ & $16(1)$ \\
$\mathrm{C}(4)$ & $1315(2)$ & $1985(2)$ & $5771(1)$ & $17(1)$ \\
$\mathrm{C}(5)$ & $752(2)$ & $1704(2)$ & $6245(1)$ & $17(1)$ \\
$\mathrm{C}(6)$ & $-20(2)$ & $1628(2)$ & $6028(1)$ & $12(1)$ \\
$\mathrm{C}(7)$ & $-229(2)$ & $1795(2)$ & $5353(1)$ & $12(1)$ \\
$\mathrm{C}(8)$ & $-538(1)$ & $2030(2)$ & $3870(1)$ & $16(1)$ \\
$\mathrm{C}(9)$ & $-791(1)$ & $2695(2)$ & $3324(1)$ & $20(1)$ \\
$\mathrm{C}(10)$ & $-1490(1)$ & $2519(2)$ & $2985(1)$ & $16(1)$ \\
$\mathrm{C}(11)$ & $-1967(2)$ & $1668(2)$ & $3175(1)$ & $17(1)$ \\
$\mathrm{C}(12)$ & $-1714(2)$ & $964(2)$ & $3685(1)$ & $36(1)$ \\
$\mathrm{C}(13)$ & $-1016(2)$ & $1134(2)$ & $4025(1)$ & $33(1)$ \\
$\mathrm{C}(14)$ & $2309(2)$ & $1248(2)$ & $4214(1)$ & $21(1)$ \\
$\mathrm{C}(15)$ & $1709(2)$ & $516(3)$ & $3878(2)$ & $13(1)$ \\
$\mathrm{C}(16)$ & $2674(2)$ & $701(3)$ & $4831(1)$ & $21(1)$ \\
$\mathrm{C}(17)$ & $2940(2)$ & $1592(2)$ & $3709(1)$ & $19(1)$ \\
$\mathrm{C}(18)$ & $-347(1)$ & $5004(2)$ & $3338(1)$ & \\
$\mathrm{C}(19)$ & $-70(2)$ & $5098(2)$ & $4072(1)$ & \\
$\mathrm{C}(20)$ & $-1228(2)$ & $5181(2)$ & $3291(1)$ & \\
& $94(2)$ & $5779(2)$ & $2862(1)$ & \\
\hline
\end{tabular}


Table 13. Bond lengths $[\AA]$ and angles $\left[^{\circ}\right]$ for 4 .

\begin{tabular}{|c|c|c|c|}
\hline$\overline{\mathrm{Cu}-\mathrm{N}}$ & $1.915(2)$ & $\mathrm{N}-\mathrm{Cu}-\mathrm{Cl}$ & $173.44(7)$ \\
\hline $\mathrm{Cu}-\mathrm{Cl}$ & $2.2068(7)$ & $\mathrm{N}-\mathrm{Cu}-\mathrm{S}(2)$ & $86.37(6)$ \\
\hline $\mathrm{Cu}-\mathrm{S}(2)$ & $2.3465(7)$ & $\mathrm{Cl}-\mathrm{Cu}-\mathrm{S}(2)$ & $92.80(2)$ \\
\hline $\mathrm{Cu}-\mathrm{S}(1)$ & $2.3476(7)$ & $\mathrm{N}-\mathrm{Cu}-\mathrm{S}(1)$ & $86.02(6)$ \\
\hline $\mathrm{N}-\mathrm{C}(1)$ & $1.381(3)$ & $\mathrm{Cl}-\mathrm{Cu}-\mathrm{S}(1)$ & $95.89(2)$ \\
\hline $\mathrm{N}-\mathrm{C}(7)$ & $1.399(3)$ & $\mathrm{S}(2)-\mathrm{Cu}-\mathrm{S}(1)$ & $167.35(3)$ \\
\hline$S(1)-C(2)$ & $1.770(2)$ & $\mathrm{C}(1)-\mathrm{N}-\mathrm{C}(7)$ & $122.3(2)$ \\
\hline $\mathrm{S}(1)-\mathrm{C}(13)$ & $1.875(3)$ & $\mathrm{C}(1)-\mathrm{N}-\mathrm{Cu}$ & $119.69(16)$ \\
\hline $\mathrm{S}(2)-\mathrm{C}(8)$ & $1.773(2)$ & $\mathrm{C}(7)-\mathrm{N}-\mathrm{Cu}$ & $117.76(16)$ \\
\hline$S(2)-C(17)$ & $1.872(3)$ & $\mathrm{C}(2)-\mathrm{S}(1)-\mathrm{C}(13)$ & $104.15(12)$ \\
\hline$C(1)-C(6)$ & $1.410(3)$ & $\mathrm{C}(2)-\mathrm{S}(1)-\mathrm{Cu}$ & $94.75(8)$ \\
\hline$C(1)-C(2)$ & $1.419(3)$ & $\mathrm{C}(13)-\mathrm{S}(1)-\mathrm{Cu}$ & $105.87(8)$ \\
\hline$C(2)-C(3)$ & $1.391(3)$ & $\mathrm{C}(8)-\mathrm{S}(2)-\mathrm{C}(17)$ & $107.67(11)$ \\
\hline$C(3)-C(4)$ & $1.380(3)$ & $\mathrm{C}(8)-\mathrm{S}(2)-\mathrm{Cu}$ & $94.73(8)$ \\
\hline $\mathrm{C}(3)-\mathrm{H}(3)$ & 0.9500 & $\mathrm{C}(17)-\mathrm{S}(2)-\mathrm{Cu}$ & $104.55(8)$ \\
\hline$C(4)-C(5)$ & $1.388(4)$ & $\mathrm{N}-\mathrm{C}(1)-\mathrm{C}(6)$ & $125.8(2)$ \\
\hline $\mathrm{C}(4)-\mathrm{H}(4)$ & 0.9500 & $\mathrm{~N}-\mathrm{C}(1)-\mathrm{C}(2)$ & $118.3(2)$ \\
\hline$C(5)-C(6)$ & $1.385(3)$ & $\mathrm{C}(6)-\mathrm{C}(1)-\mathrm{C}(2)$ & $115.7(2)$ \\
\hline $\mathrm{C}(5)-\mathrm{H}(5)$ & 0.9500 & $\mathrm{C}(3)-\mathrm{C}(2)-\mathrm{C}(1)$ & $122.0(2)$ \\
\hline $\mathrm{C}(6)-\mathrm{H}(6)$ & 0.9500 & $\mathrm{C}(3)-\mathrm{C}(2)-\mathrm{S}(1)$ & $119.94(19)$ \\
\hline$C(7)-C(12)$ & $1.406(3)$ & $\mathrm{C}(1)-\mathrm{C}(2)-\mathrm{S}(1)$ & $117.97(18)$ \\
\hline$C(7)-C(8)$ & $1.413(3)$ & $\mathrm{C}(4)-\mathrm{C}(3)-\mathrm{C}(2)$ & $120.8(2)$ \\
\hline $\mathrm{C}(8)-\mathrm{C}(9)$ & $1.384(3)$ & $\mathrm{C}(4)-\mathrm{C}(3)-\mathrm{H}(3)$ & 119.6 \\
\hline$C(9)-C(10)$ & $1.378(4)$ & $\mathrm{C}(2)-\mathrm{C}(3)-\mathrm{H}(3)$ & 119.6 \\
\hline $\mathrm{C}(9)-\mathrm{H}(11)$ & 0.9500 & $\mathrm{C}(3)-\mathrm{C}(4)-\mathrm{C}(5)$ & $118.2(2)$ \\
\hline$C(10)-C(11)$ & $1.391(4)$ & $\mathrm{C}(3)-\mathrm{C}(4)-\mathrm{H}(4)$ & 120.9 \\
\hline $\mathrm{C}(10)-\mathrm{H}(10)$ & 0.9500 & $\mathrm{C}(5)-\mathrm{C}(4)-\mathrm{H}(4)$ & 120.9 \\
\hline$C(11)-C(12)$ & $1.383(3)$ & $C(6)-C(5)-C(4)$ & $121.7(2)$ \\
\hline $\mathrm{C}(11)-\mathrm{H}(9)$ & 0.9500 & $\mathrm{C}(6)-\mathrm{C}(5)-\mathrm{H}(5)$ & 119.1 \\
\hline $\mathrm{C}(12)-\mathrm{H}(8)$ & 0.9500 & $\mathrm{C}(4)-\mathrm{C}(5)-\mathrm{H}(5)$ & 119.1 \\
\hline$C(13)-C(14)$ & $1.516(4)$ & $C(5)-C(6)-C(1)$ & $121.4(2)$ \\
\hline$C(13)-C(15)$ & $1.518(4)$ & $\mathrm{C}(5)-\mathrm{C}(6)-\mathrm{H}(6)$ & 119.3 \\
\hline$C(13)-C(16)$ & $1.522(3)$ & $\mathrm{C}(1)-\mathrm{C}(6)-\mathrm{H}(6)$ & 119.3 \\
\hline $\mathrm{C}(14)-\mathrm{H}(18 \mathrm{~A})$ & 0.9800 & $\mathrm{~N}-\mathrm{C}(7)-\mathrm{C}(12)$ & $125.2(2)$ \\
\hline $\mathrm{C}(14)-\mathrm{H}(18 \mathrm{~B})$ & 0.9800 & $\mathrm{~N}-\mathrm{C}(7)-\mathrm{C}(8)$ & $118.3(2)$ \\
\hline $\mathrm{C}(14)-\mathrm{H}(18 \mathrm{C})$ & 0.9800 & $\mathrm{C}(12)-\mathrm{C}(7)-\mathrm{C}(8)$ & $116.2(2)$ \\
\hline $\mathrm{C}(15)-\mathrm{H}(17 \mathrm{~A})$ & 0.9800 & $\mathrm{C}(9)-\mathrm{C}(8)-\mathrm{C}(7)$ & $122.4(2)$ \\
\hline $\mathrm{C}(15)-\mathrm{H}(17 \mathrm{~B})$ & 0.9800 & $\mathrm{C}(9)-\mathrm{C}(8)-\mathrm{S}(2)$ & $118.94(19)$ \\
\hline $\mathrm{C}(15)-\mathrm{H}(17 \mathrm{C})$ & 0.9800 & $\mathrm{C}(7)-\mathrm{C}(8)-\mathrm{S}(2)$ & $117.88(18)$ \\
\hline $\mathrm{C}(16)-\mathrm{H}(19 \mathrm{~A})$ & 0.9800 & $\mathrm{C}(10)-\mathrm{C}(9)-\mathrm{C}(8)$ & $120.0(2)$ \\
\hline $\mathrm{C}(16)-\mathrm{H}(19 \mathrm{~B})$ & 0.9800 & $\mathrm{C}(10)-\mathrm{C}(9)-\mathrm{H}(11)$ & 120.0 \\
\hline $\mathrm{C}(16)-\mathrm{H}(19 \mathrm{C})$ & 0.9800 & $\mathrm{C}(8)-\mathrm{C}(9)-\mathrm{H}(11)$ & 120.0 \\
\hline$C(17)-C(18)$ & $1.519(3)$ & $C(9)-C(10)-C(11)$ & $119.0(2)$ \\
\hline$C(17)-C(19)$ & $1.523(3)$ & $\mathrm{C}(9)-\mathrm{C}(10)-\mathrm{H}(10)$ & 120.5 \\
\hline$C(17)-C(20)$ & $1.532(3)$ & $\mathrm{C}(11)-\mathrm{C}(10)-\mathrm{H}(10)$ & 120.5 \\
\hline $\mathrm{C}(18)-\mathrm{H}(13 \mathrm{~A})$ & 0.9800 & $C(12)-C(11)-C(10)$ & $121.3(3)$ \\
\hline $\mathrm{C}(18)-\mathrm{H}(13 \mathrm{~B})$ & 0.9800 & $\mathrm{C}(12)-\mathrm{C}(11)-\mathrm{H}(9)$ & 119.4 \\
\hline $\mathrm{C}(18)-\mathrm{H}(13 \mathrm{C})$ & 0.9800 & $\mathrm{C}(10)-\mathrm{C}(11)-\mathrm{H}(9)$ & 119.4 \\
\hline $\mathrm{C}(19)-\mathrm{H}(15 \mathrm{~A})$ & 0.9800 & $\mathrm{C}(11)-\mathrm{C}(12)-\mathrm{C}(7)$ & $121.0(2)$ \\
\hline $\mathrm{C}(19)-\mathrm{H}(15 \mathrm{~B})$ & 0.9800 & $\mathrm{C}(11)-\mathrm{C}(12)-\mathrm{H}(8)$ & 119.5 \\
\hline $\mathrm{C}(19)-\mathrm{H}(15 \mathrm{C})$ & 0.9800 & $\mathrm{C}(7)-\mathrm{C}(12)-\mathrm{H}(8)$ & 119.5 \\
\hline $\mathrm{C}(20)-\mathrm{H}(14 \mathrm{~A})$ & 0.9800 & $C(14)-C(13)-C(15)$ & 111.1(3) \\
\hline $\mathrm{C}(20)-\mathrm{H}(14 \mathrm{~B})$ & 0.9800 & $\mathrm{C}(14)-\mathrm{C}(13)-\mathrm{C}(16)$ & $111.3(2)$ \\
\hline $\mathrm{C}(20)-\mathrm{H}(14 \mathrm{C})$ & 0.9800 & $C(15)-C(13)-C(16)$ & $110.5(2)$ \\
\hline
\end{tabular}




$\begin{array}{ll}\mathrm{C}(14)-\mathrm{C}(13)-\mathrm{S}(1) & 110.54(18) \\ \mathrm{C}(15)-\mathrm{C}(13)-\mathrm{S}(1) & 108.59(18) \\ \mathrm{C}(16)-\mathrm{C}(13)-\mathrm{S}(1) & 104.67(18) \\ \mathrm{C}(13)-\mathrm{C}(14)-\mathrm{H}(18 \mathrm{~A}) & 109.5 \\ \mathrm{C}(13)-\mathrm{C}(14)-\mathrm{H}(18 \mathrm{~B}) & 109.5 \\ \mathrm{H}(18 \mathrm{~A})-\mathrm{C}(14)-\mathrm{H}(18 \mathrm{~B}) & 109.5 \\ \mathrm{C}(13)-\mathrm{C}(14)-\mathrm{H}(18 \mathrm{C}) & 109.5 \\ \mathrm{H}(18 \mathrm{~A})-\mathrm{C}(14)-\mathrm{H}(18 \mathrm{C}) & 109.5 \\ \mathrm{H}(18 \mathrm{~B})-\mathrm{C}(14)-\mathrm{H}(18 \mathrm{C}) & 109.5 \\ \mathrm{C}(13)-\mathrm{C}(15)-\mathrm{H}(17 \mathrm{~A}) & 109.5 \\ \mathrm{C}(13)-\mathrm{C}(15)-\mathrm{H}(17 \mathrm{~B}) & 109.5 \\ \mathrm{H}(17 \mathrm{~A})-\mathrm{C}(15)-\mathrm{H}(17 \mathrm{~B}) & 109.5 \\ \mathrm{C}(13)-\mathrm{C}(15)-\mathrm{H}(17 \mathrm{C}) & 109.5 \\ \mathrm{H}(17 \mathrm{~A})-\mathrm{C}(15)-\mathrm{H}(17 \mathrm{C}) & 109.5 \\ \mathrm{H}(17 \mathrm{~B})-\mathrm{C}(15)-\mathrm{H}(17 \mathrm{C}) & 109.5 \\ \mathrm{C}(13)-\mathrm{C}(16)-\mathrm{H}(19 \mathrm{~A}) & 109.5 \\ \mathrm{C}(13)-\mathrm{C}(16)-\mathrm{H}(19 \mathrm{~B}) & 109.5 \\ \mathrm{H}(19 \mathrm{~A})-\mathrm{C}(16)-\mathrm{H}(19 \mathrm{~B}) & 109.5 \\ \mathrm{C}(13)-\mathrm{C}(16)-\mathrm{H}(19 \mathrm{C}) & 109.5 \\ \mathrm{H}(19 \mathrm{~A})-\mathrm{C}(16)-\mathrm{H}(19 \mathrm{C}) & 109.5 \\ \mathrm{H}(19 \mathrm{~B})-\mathrm{C}(16)-\mathrm{H}(19 \mathrm{C}) & 109.5 \\ \mathrm{C}(18)-\mathrm{C}(17)-\mathrm{C}(19) & 110.8(2) \\ \mathrm{C}(18)-\mathrm{C}(17)-\mathrm{C}(20) & 112.0(2)\end{array}$

$\begin{array}{ll}\mathrm{C}(19)-\mathrm{C}(17)-\mathrm{C}(20) & 111.1(2) \\ \mathrm{C}(18)-\mathrm{C}(17)-\mathrm{S}(2) & 110.97(18) \\ \mathrm{C}(19)-\mathrm{C}(17)-\mathrm{S}(2) & 108.59(18) \\ \mathrm{C}(20)-\mathrm{C}(17)-\mathrm{S}(2) & 103.12(17) \\ \mathrm{C}(17)-\mathrm{C}(18)-\mathrm{H}(13 \mathrm{~A}) & 109.5 \\ \mathrm{C}(17)-\mathrm{C}(18)-\mathrm{H}(13 \mathrm{~B}) & 109.5 \\ \mathrm{H}(13 \mathrm{~A})-\mathrm{C}(18)-\mathrm{H}(13 \mathrm{~B}) & 109.5 \\ \mathrm{C}(17)-\mathrm{C}(18)-\mathrm{H}(13 \mathrm{C}) & 109.5 \\ \mathrm{H}(13 \mathrm{~A})-\mathrm{C}(18)-\mathrm{H}(13 \mathrm{C}) & 109.5 \\ \mathrm{H}(13 \mathrm{~B})-\mathrm{C}(18)-\mathrm{H}(13 \mathrm{C}) & 109.5 \\ \mathrm{C}(17)-\mathrm{C}(19)-\mathrm{H}(15 \mathrm{~A}) & 109.5 \\ \mathrm{C}(17)-\mathrm{C}(19)-\mathrm{H}(15 \mathrm{~B}) & 109.5 \\ \mathrm{H}(15 \mathrm{~A})-\mathrm{C}(19)-\mathrm{H}(15 \mathrm{~B}) & 109.5 \\ \mathrm{C}(17)-\mathrm{C}(19)-\mathrm{H}(15 \mathrm{C}) & 109.5 \\ \mathrm{H}(15 \mathrm{~A})-\mathrm{C}(19)-\mathrm{H}(15 \mathrm{C}) & 109.5 \\ \mathrm{H}(15 \mathrm{~B})-\mathrm{C}(19)-\mathrm{H}(15 \mathrm{C}) & 109.5 \\ \mathrm{C}(17)-\mathrm{C}(20)-\mathrm{H}(14 \mathrm{~A}) & 109.5 \\ \mathrm{C}(17)-\mathrm{C}(20)-\mathrm{H}(14 \mathrm{~B}) & 109.5 \\ \mathrm{H}(14 \mathrm{~A})-\mathrm{C}(20)-\mathrm{H}(14 \mathrm{~B}) & 109.5 \\ \mathrm{C}(17)-\mathrm{C}(20)-\mathrm{H}(14 \mathrm{C}) & 109.5 \\ \mathrm{H}(14 \mathrm{~A})-\mathrm{C}(20)-\mathrm{H}(14 \mathrm{C}) & 109.5 \\ \mathrm{H}(14 \mathrm{~B})-\mathrm{C}(20)-\mathrm{H}(14 \mathrm{C}) & 109.5 \\ & \end{array}$

Symmetry transformations used to generate equivalent atoms: 
Table 14. Anisotropic displacement parameters $\left(\AA^{2} \times 10^{3}\right)$ for 4. The anisotropic displacement factor exponent takes the form: $-2 \pi^{2}\left[h^{2} a^{* 2} U^{11}+\ldots+2 h k a^{*} b^{*} U^{12}\right]$

\begin{tabular}{lcccccc}
\hline & $\mathrm{U}^{11}$ & $\mathrm{U}^{22}$ & $\mathrm{U}^{33}$ & $\mathrm{U}^{23}$ & $\mathrm{U}^{13}$ & $\mathrm{U}^{12}$ \\
\hline $\mathrm{Cu}$ & $10(1)$ & $14(1)$ & $11(1)$ & $1(1)$ & $0(1)$ & $0(1)$ \\
$\mathrm{N}$ & $11(1)$ & $20(1)$ & $11(1)$ & $2(1)$ & $0(1)$ & $-1(1)$ \\
$\mathrm{S}(1)$ & $12(1)$ & $14(1)$ & $12(1)$ & $1(1)$ & $-1(1)$ & $-1(1)$ \\
$\mathrm{S}(2)$ & $11(1)$ & $14(1)$ & $11(1)$ & $1(1)$ & $0(1)$ & $0(1)$ \\
$\mathrm{Cl}$ & $12(1)$ & $23(1)$ & $17(1)$ & $5(1)$ & $2(1)$ & $-2(1)$ \\
$\mathrm{C}(1)$ & $14(1)$ & $10(1)$ & $12(1)$ & $-2(1)$ & $-2(1)$ & $2(1)$ \\
$\mathrm{C}(2)$ & $11(1)$ & $11(1)$ & $15(1)$ & $-2(1)$ & $2(1)$ & $0(1)$ \\
$\mathrm{C}(3)$ & $15(1)$ & $16(1)$ & $17(1)$ & $-3(1)$ & $-3(1)$ & $2(1)$ \\
$\mathrm{C}(4)$ & $25(2)$ & $18(2)$ & $8(1)$ & $1(1)$ & $-2(1)$ & $2(1)$ \\
$\mathrm{C}(5)$ & $22(2)$ & $17(2)$ & $13(1)$ & $0(1)$ & $4(1)$ & $0(1)$ \\
$\mathrm{C}(6)$ & $14(1)$ & $15(2)$ & $16(1)$ & $0(1)$ & $0(1)$ & $1(1)$ \\
$\mathrm{C}(7)$ & $9(1)$ & $17(1)$ & $9(1)$ & $-2(1)$ & $2(1)$ & $1(1)$ \\
$\mathrm{C}(8)$ & $13(1)$ & $13(1)$ & $11(1)$ & $-2(1)$ & $3(1)$ & $-2(1)$ \\
$\mathrm{C}(9)$ & $13(1)$ & $21(2)$ & $13(1)$ & $2(1)$ & $-1(1)$ & $0(1)$ \\
$\mathrm{C}(10)$ & $13(1)$ & $30(2)$ & $19(2)$ & $0(1)$ & $-3(1)$ & $-6(1)$ \\
$\mathrm{C}(11)$ & $19(1)$ & $24(2)$ & $18(1)$ & $2(1)$ & $3(1)$ & $-9(1)$ \\
$\mathrm{C}(12)$ & $17(1)$ & $18(2)$ & $14(1)$ & $3(1)$ & $2(1)$ & $-1(1)$ \\
$\mathrm{C}(13)$ & $16(1)$ & $15(2)$ & $22(1)$ & $0(1)$ & $5(1)$ & $3(1)$ \\
$\mathrm{C}(14)$ & $22(2)$ & $27(2)$ & $60(2)$ & $-22(2)$ & $12(2)$ & $-3(1)$ \\
$\mathrm{C}(15)$ & $39(2)$ & $33(2)$ & $27(2)$ & $12(1)$ & $13(1)$ & $23(2)$ \\
$\mathrm{C}(16)$ & $16(1)$ & $25(2)$ & $24(2)$ & $3(1)$ & $7(1)$ & $5(1)$ \\
$\mathrm{C}(17)$ & $13(1)$ & $10(1)$ & $17(1)$ & $-1(1)$ & $0(1)$ & $3(1)$ \\
$\mathrm{C}(18)$ & $26(2)$ & $18(2)$ & $19(2)$ & $-4(1)$ & $1(1)$ & $1(1)$ \\
$\mathrm{C}(19)$ & $17(1)$ & $17(2)$ & $33(2)$ & $-3(1)$ & $0(1)$ & $4(1)$ \\
$\mathrm{C}(20)$ & $20(1)$ & $14(2)$ & $24(2)$ & $3(1)$ & $2(1)$ & $-2(1)$ \\
& & & & & & \\
\hline & & & & & & \\
\end{tabular}


Table 15. Hydrogen coordinates ( $\left.\mathrm{x} 10^{4}\right)$ and isotropic displacement parameters $\left(\AA^{2} \times 10^{3}\right)$ for 4 .

\begin{tabular}{lrrrl}
\hline & $\mathrm{x}$ & $\mathrm{y}$ & $\mathrm{z}$ & $\mathrm{U}_{\mathrm{eq}}$ \\
\hline & & & & \\
$\mathrm{H}(3)$ & 1843 & 2073 & 5912 & 19 \\
$\mathrm{H}(4)$ & 890 & 1567 & 6707 & 21 \\
$\mathrm{H}(5)$ & -415 & 1456 & 6352 & 21 \\
$\mathrm{H}(6)$ & -764 & 1744 & 5225 & 18 \\
$\mathrm{H}(11)$ & -1642 & 2986 & 2622 & 19 \\
$\mathrm{H}(10)$ & -2460 & 1564 & 2961 & 24 \\
$\mathrm{H}(9)$ & -2028 & 354 & 3801 & 24 \\
$\mathrm{H}(8)$ & -856 & 638 & 4370 & 20 \\
$\mathrm{H}(18 \mathrm{~A})$ & 1956 & -172 & 3750 & 54 \\
$\mathrm{H}(18 \mathrm{~B})$ & 1502 & 872 & 3467 & 54 \\
$\mathrm{H}(18 \mathrm{C})$ & 1279 & 376 & 4198 & 54 \\
$\mathrm{H}(17 \mathrm{~A})$ & 2969 & 60 & 4683 & 50 \\
$\mathrm{H}(17 \mathrm{~B})$ & 2261 & 479 & 5149 & 50 \\
$\mathrm{H}(17 \mathrm{C})$ & 3028 & 1210 & 5060 & 50 \\
$\mathrm{H}(19 \mathrm{~A})$ & 3338 & 2022 & 3947 & 32 \\
$\mathrm{H}(19 \mathrm{~B})$ & 2704 & 2030 & 3346 & 32 \\
$\mathrm{H}(19 \mathrm{C})$ & 3184 & 946 & 3510 & 32 \\
$\mathrm{H}(13 \mathrm{~A})$ & -207 & 5816 & 4252 & 32 \\
$\mathrm{H}(13 \mathrm{~B})$ & 500 & 5003 & 4090 & 32 \\
$\mathrm{H}(13 \mathrm{C})$ & -322 & 4536 & 4350 & 32 \\
$\mathrm{H}(15 \mathrm{~A})$ & -1356 & 5917 & 3445 & 33 \\
$\mathrm{H}(15 \mathrm{~B})$ & -1498 & 4650 & 3580 & 33 \\
$\mathrm{H}(15 \mathrm{C})$ & -1398 & 5089 & 2816 & 33 \\
$\mathrm{H}(14 \mathrm{~A})$ & -127 & 5732 & 2401 & 29 \\
$\mathrm{H}(14 \mathrm{~B})$ & 649 & 5576 & 2849 & 29 \\
$\mathrm{H}(14 \mathrm{C})$ & 43 & 6524 & 3032 & 29 \\
& & & & \\
\hline
\end{tabular}

